\title{
An evaluation of vibration and other effects on the accuracy of grip and push force recall
}

Thomas W. McDowell

Follow this and additional works at: https://researchrepository.wvu.edu/etd

\section{Recommended Citation}

McDowell, Thomas W., "An evaluation of vibration and other effects on the accuracy of grip and push force recall" (2006). Graduate Theses, Dissertations, and Problem Reports. 3447.

https://researchrepository.wvu.edu/etd/3447

This Dissertation is protected by copyright and/or related rights. It has been brought to you by the The Research Repository @ WVU with permission from the rights-holder(s). You are free to use this Dissertation in any way that is permitted by the copyright and related rights legislation that applies to your use. For other uses you must obtain permission from the rights-holder(s) directly, unless additional rights are indicated by a Creative Commons license in the record and/ or on the work itself. This Dissertation has been accepted for inclusion in WVU Graduate Theses, Dissertations, and Problem Reports collection by an authorized administrator of The Research Repository @ WVU.

For more information, please contact researchrepository@mail.wvu.edu. 


\title{
AN EVALUATION OF VIBRATION AND OTHER EFFECTS ON THE ACCURACY OF GRIP AND PUSH FORCE RECALL
}

\author{
by
}

Thomas W. McDowell

\author{
Dissertation submitted to \\ College of Engineering and Mineral Resources \\ at West Virginia University \\ in partial fulfillment of the requirements \\ for the degree of
}

Doctor of Philosophy

in

Occupational Safety and Health

\section{Committee:}

Steven F. Wiker, Ph.D., Committee Chair

Ren G. Dong, Ph.D.

Steven E. Guffey, Ph.D.

Warren R. Myers, Ph.D.

Gary L. Winn, Ph.D.

Department of Industrial and Management Systems Engineering

$$
\begin{gathered}
\text { Morgantown, West Virginia } \\
2006
\end{gathered}
$$

Copyright 2006 Thomas W. McDowell 


\title{
ABSTRACT \\ AN EVALUATION OF VIBRATION AND OTHER EFFECTS ON THE ACCURACY OF GRIP AND PUSH FORCE RECALL
}

\author{
by Thomas W. McDowell
}

Operators of vibratory hand tools can be at risk of developing health problems associated with repeated forceful actions and exposure to intense hand-transmitted vibration. To better assess health risks, comprehensive risk evaluations of these tasks must include quantitative assessments of hand-tool coupling forces. Researchers have used instrumentation for such measurements; but those techniques may be ill-suited for certain field environments. Psychophysical force-recall techniques have been proposed as alternatives to handle instrumentation. This study comprised two experiments that examined the effects of vibration and other factors upon force-recall accuracy and reliability. In each experiment, participants applied specific grip and push forces to an instrumented handle mounted on a shaker system. Participants were exposed to sinusoidal vibration at frequencies that ranged from $0 \mathrm{~Hz}$ to $250 \mathrm{~Hz}$. Three levels of applied force (grip $=30 \pm 15 \mathrm{~N}$; push $=50 \pm 25 \mathrm{~N}$ ) and two levels of vibration magnitude were examined. During the vibration exposure period, participants were provided with visual feedback while they attempted to "memorize" their applied grip and push forces. Following vibration exposure and a rest period, participants tried to duplicate the hand forces without visual feedback. Vibration frequencies, magnitudes, and hand force levels were randomized from trial to trial. To evaluate test-retest reliability, the test was repeated on a later day with each participant. Participants overestimated grip and push forces. Depending on exposure conditions, error means ranged from $2 \mathrm{~N}$ to $10 \mathrm{~N}$. The ANOVA revealed that force-recall errors for exposures between $31.5 \mathrm{~Hz}$ and $63 \mathrm{~Hz}$ were significantly higher than those at other vibration frequencies $(p<0.05)$. Errors were greater when participants were exposed to the higher vibration magnitude (mean $=9.1 \mathrm{~N}$, $95 \%$ C.I. $=8.1-10.2 \mathrm{~N}$ ) when compared with the lower vibration magnitude (mean $=$ $4.9 \mathrm{~N}, 95 \%$ C.I. $=4.0-5.7 \mathrm{~N})(p<0.05)$. The average error for females $(4.9 \mathrm{~N}, 95 \%$ C.I. $=3.9-5.9 \mathrm{~N})$ was significantly less than that for males $(8.3 \mathrm{~N}, 95 \%$ C.I. $=7.5-9.1 \mathrm{~N})(p<$ $0.05)$. The effects of force level were mixed. This method demonstrated strong testretest reliability as correlations for all but one participant were found to be significant $(0.45<r<0.95, p<0.01)$. Overall, recalled force errors were relatively small over the range of operationally-relevant hand-handle coupling forces and vibration exposure conditions. This force-recall technique shows promise as an alternative to expensive and fragile force-sensing instrumentation. 
This dissertation is dedicated to my wife, Sherry Vincent McDowell. Without her love, unconditional support, and never-ending patience, this work would have never been completed. 


\section{ACKNOWLEDGEMENTS}

I would like to thank my parents, Emily McDowell and Murvyn McDowell for establishing high standards, for leading by example, and for offering love and constant encouragement throughout my academic and professional endeavors.

I would like to express my sincere gratitude to my advisor, Dr. Steven Wiker for setting a high bar and providing the necessary support to facilitate my success in this incredible learning experience. Dr. Wiker has never hesitated to share his time, knowledge, and guidance. I feel fortunate that Dr. Wiker's positive influence will be reflected in all my future works.

I wish to thank Dr. Ren Dong for taking me under his wing and imparting his expert guidance and leadership. Dr. Dong pointed me toward this area of research, and then proceeded to grant unwavering support. I am proud to call Dr. Dong my mentor and my friend.

Special thanks are due to Dr. Steven Guffey not just for his excellent scholastic tutoring, but also for his real-world counsel. Dr. Guffey's penchant for sharing his life experiences--good and bad--is invaluable.

I would like to thank Dr. Gary Winn for his dedication to the OSH Doc program. His work helps ensure that the degree is, and will remain, meaningful in the realm of occupational safety and health and beyond. Dr. Winn was instrumental in ensuring that I met all program requirements and that all my i's were dotted and my t's were crossed throughout my doctoral studies.

I am obliged to Dr. Warren Myers for his teachings, generous support, and valuable commentary throughout my graduate work. Dr. Myers' knowledge and experience are tremendous assets to West Virginia University's IMSE programs, and I am glad I was able to take advantage of them.

My thanks go out to my colleague, Mr. Daniel Welcome, for his instrumentation development, test setup, and other collaborative efforts.

Thanks also to Mr. Chris Warren, Ms. Marissa Smith, Ms. Selina Pan, and Mr. Bryan Washington for their help in conducting these experiments.

Thanks to all human subjects who participated in these experiments. Without your interest and efforts, this research would not exist.

Finally, I wish to thank my employer, the National Institute for Occupational Safety \& Health, for providing financial and logistical support for my coursework and research. I want to thank Dr. Al Munson for his support and encouragement. I especially want to thank my supervisor, Dr. Aaron Schopper, for his trust and support. I would not have been able to achieve my academic goals without Dr. Schopper's backing. 


\section{TABLE OF CONTENTS}

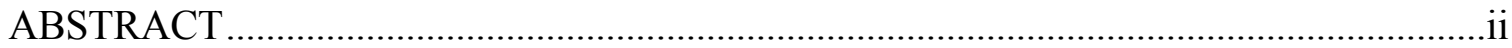

ACKNOWLEDGEMENTS ……………………………….........................................

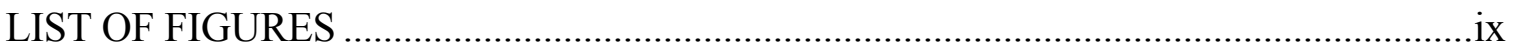

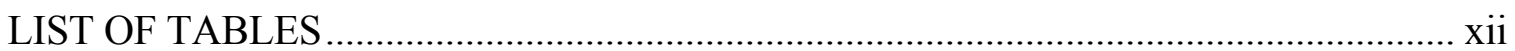

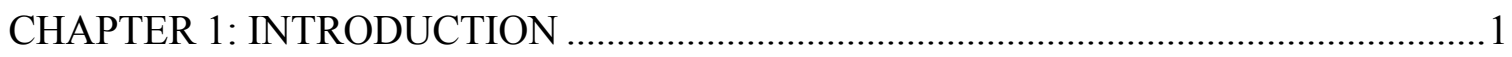

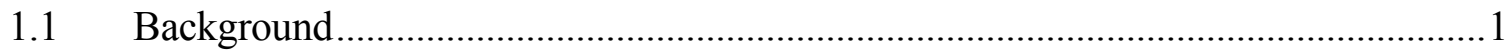

$1.2 \quad$ Objectives and hypotheses .............................................................................

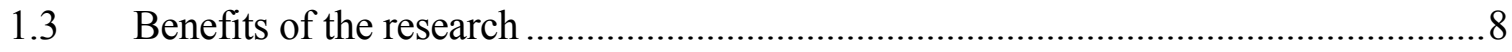

CHAPTER 2: REVIEW OF LITERATURE..................................................................

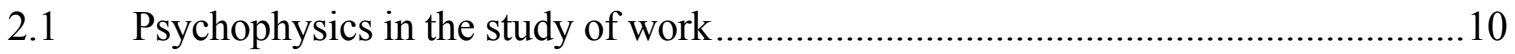

2.2 Perception and recall of movement and manual forces.............................................17

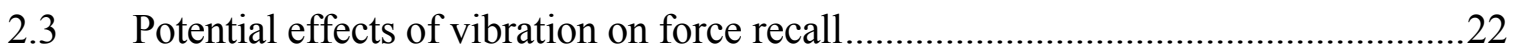

2.3.1 Vibration frequencies of concern........................................................................22

2.3.2 Vibration effects on efferent and afferent signals ...............................................23

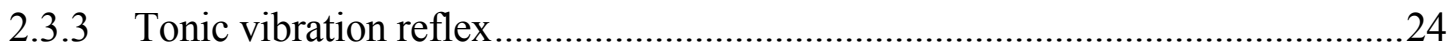

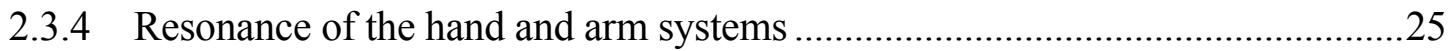

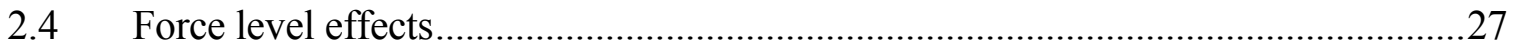

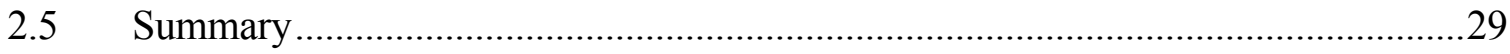

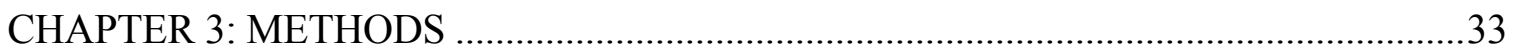

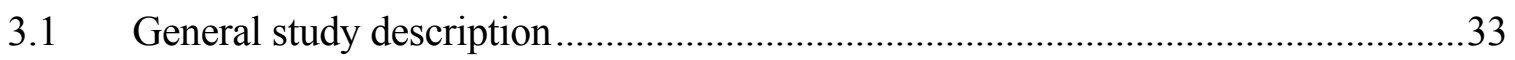

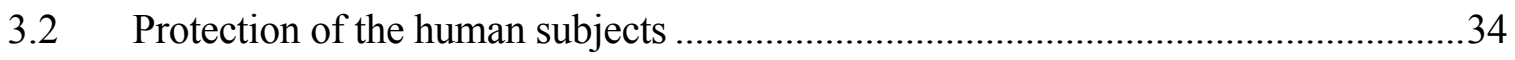

3.2.1 Protocol IRB approval / study location ………………........................................34

3.2.2 Subject recruitment and reimbursement ...........................................................34 


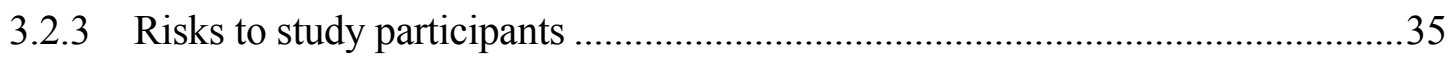

3.2.4 Informed consent procedures .............................................................................

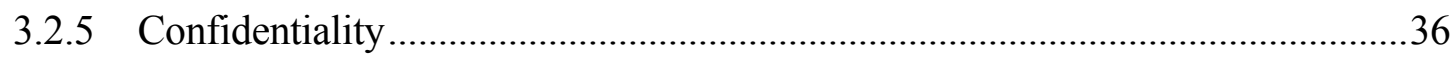

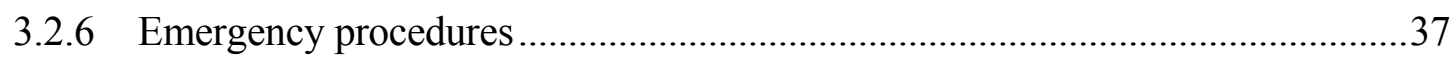

3.3 Common setup and strategy for the two experiments ……………………….........37

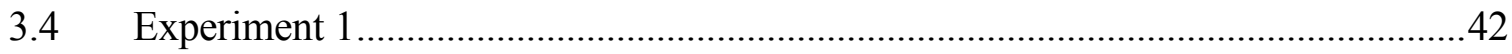

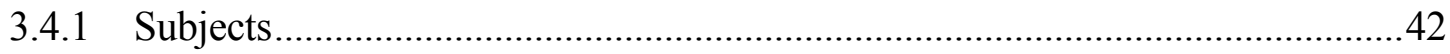

3.4.2 Vibration exposure conditions for Experiment 1 .............................................42

3.4.3 Experiment 1 Procedure .............................................................................4

3.4.4 Experiment 1 Data Analysis ........................................................................4

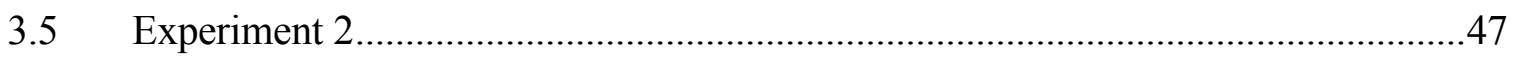

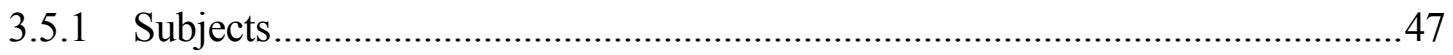

3.5.2 Vibration Exposure Conditions for Experiment 2.............................................4

3.5.3 Experiment 2 Procedure.................................................................................49

3.5.4 Experiment 2 Data Analysis ............................................................................

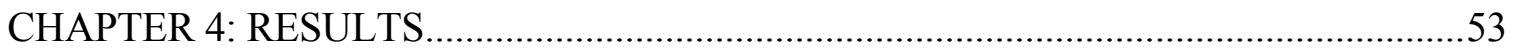

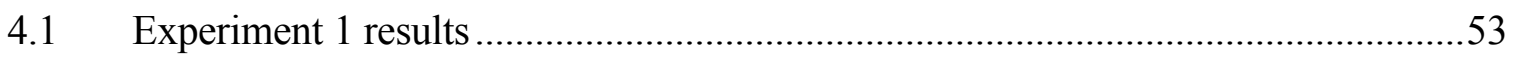

4.1.1 Subject descriptors for Experiment 1 ………………....................................53

4.1.2 Recalled grip and push force distributions for Experiment 1 .............................54

4.1.3 Analysis of variance (ANOVA) for Experiment 1 ............................................56

4.1.4 Statistical power for Experiment 1 ……………………………………….....57

4.1.5 Vibration frequency effects in Experiment 1 ……….......................................59

4.1.6 Force level effects in Experiment 1 ..............................................................63

4.1.7 Test-retest reliability in Experiment 1 .............................................................64

4.1.8 Exertion type effects in Experiment 1 ............................................................65 
4.1.9 Effects of rest period duration in Experiment 1 ..............................................66

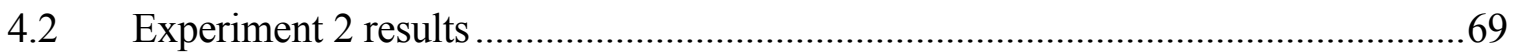

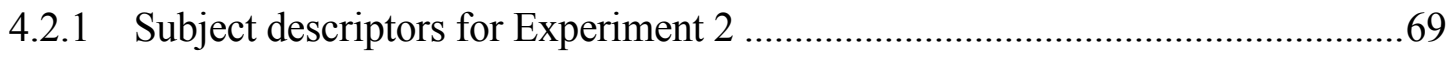

4.2.2 Recalled grip and push force distributions in Experiment 2 ........................... 70

4.2.3 Analysis of variance (ANOVA) for Experiment 2 .......................................... 72

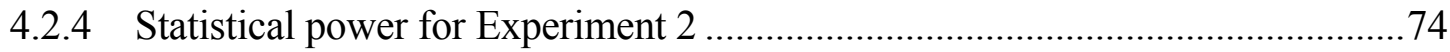

4.2.5 Vibration frequency effects in Experiment 2 .............................................. 75

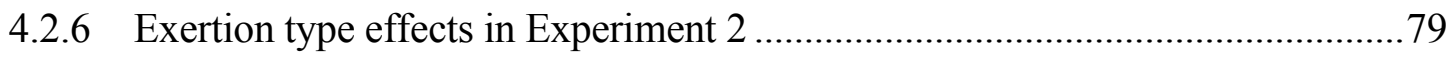

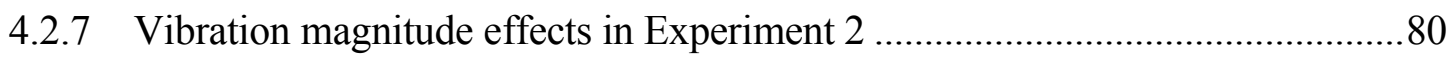

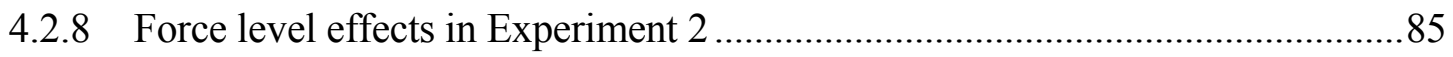

4.2.9 Test-retest reliability in Experiment 2 ....................................................... 87

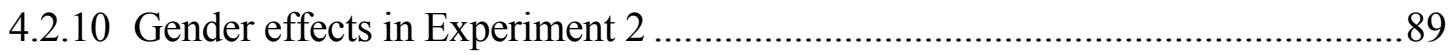

4.3 Some combined results of the two experiments ................................................. 91

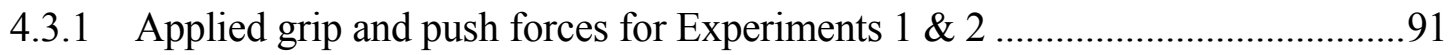

4.3.2 Recalled grip and push force distributions for Experiments $1 \& 2$..................92

4.3.3 Frequency effects found in Experiments $1 \&$ 2 .............................................94

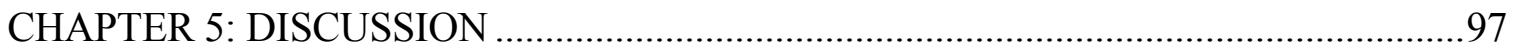

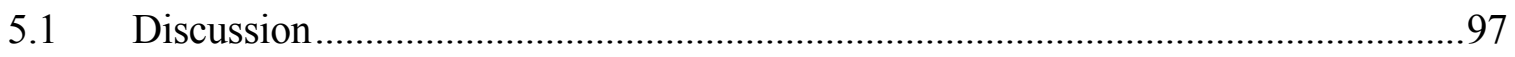

5.1.1 Control of grip and push during the force memorization period .....................97

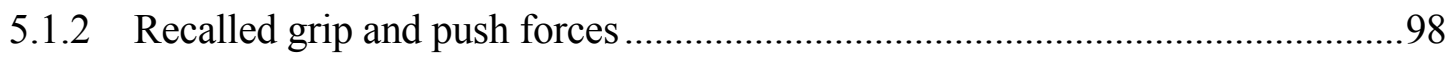

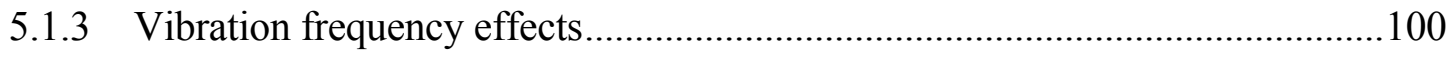

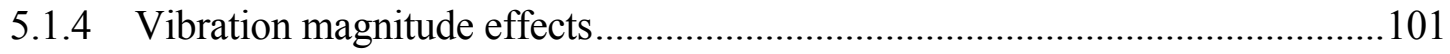

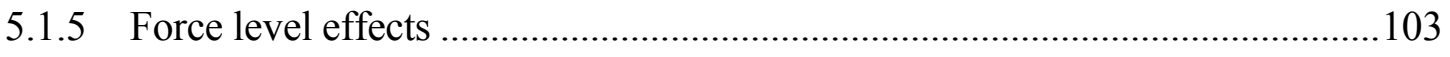

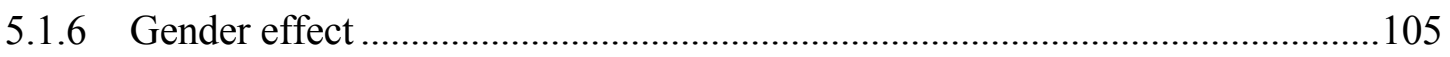

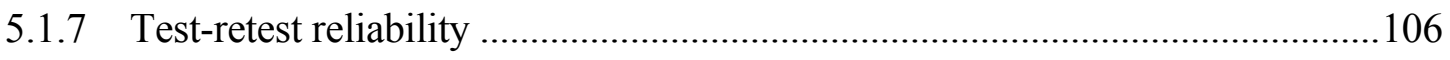




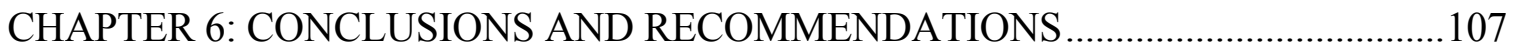

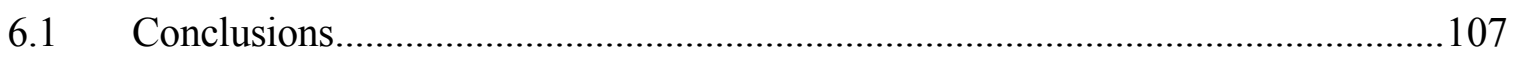

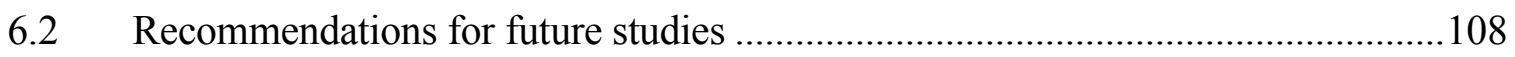

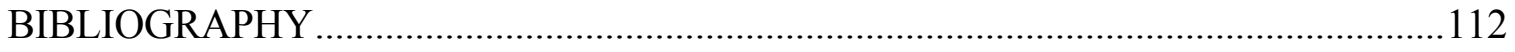

APPENDIX A: PROTOCOL APPROVAL NOTICES FROM NIOSH AND WVU .......120

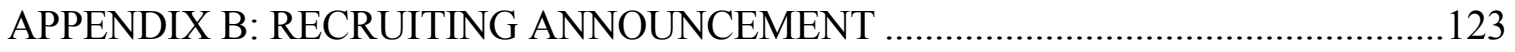

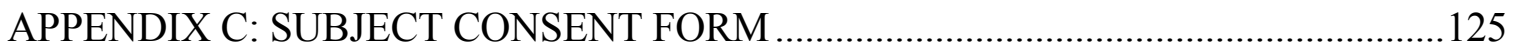

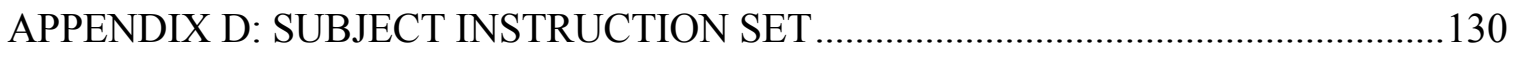

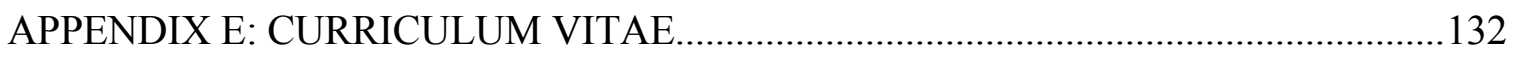




\section{LIST OF FIGURES}

Figure 3.3.1 Subject posture and experimental setup for the study.

Figure 3.3.2 Subject posture during the vibration exposure/force memorization phase of a typical trial.

Figure 3.3.3 Schematic of the instrumented handle.

Figure 3.3.4 Grip and push force feedback display on a computer monitor.

Figure 3.4.1 Timeline of events during a force memorization and recall trial in Experiment 1

Figure 3.5.1 ANSI limits of daily vibration exposure for the four vibration frequencies used in Experiment 2 (ANSI S3.34, 1986)...

Figure 3.5.2 Timeline of events during a force memorization and recall trial in Experiment 2

Figure 4.1.1 Distribution of recalled forces for (a) grip and (b) push by target force across all vibration exposure conditions of Experiment 1.

Figure 4.1.2 Experiment 1 grip and push force-recall error means plotted against vibration frequency across all force levels.

Figure 4.1.3 Experiment 1 grip and push force-recall error means plotted against vibration frequency. (Y error bars $=95 \%$ C.I.)

Figure 4.1.4 Experiment 1 grip force-recall error means for low, medium, and high target force levels plotted against vibration frequency.

Figure 4.1.5 Experiment 1 push force-recall error means for low, medium, and high target force levels plotted against vibration frequency.

Figure 4.1.6 Force-recall error versus relative grip and push force levels across all frequencies of vibration in Experiment 1.

Figure 4.1.7 Force-recall error versus relative grip and push force levels in Experiment

1. $(\mathrm{Y}$ error bars $=95 \%$ C.I. $)$

Figure 4.1.8 Force-recall error as a function of exertion type and rest period duration in Experiment 1

Figure 4.1.9 Force-recall error as a function of exertion type and rest period duration in Experiment 1. (Y error bars $=95 \%$ C.I. $)$ 
Figure 4.2.1 Distribution of recalled forces for (a) grip and (b) push by target force across all vibration exposure conditions of Experiment 2.

Figure 4.2.2 Experiment 2 grip and push force-recall error means plotted against vibration frequency across all force levels. .76

Figure 4.2.3 Experiment 2 grip and push force-recall error means plotted against vibration frequency. (Y error bars $=95 \%$ C.I.)

Figure 4.2.4 Experiment 2 grip force-recall error means for low, medium, and high target force levels plotted against vibration frequency.

Figure 4.2.5 Experiment 2 push force-recall error means for low, medium, and high target force levels plotted against vibration frequency. .78

Figure 4.2.6 Force-recall error as a function of vibration frequency and vibration magnitude across all conditions of Experiment 2.

Figure 4.2.7 Force-recall error as a function of vibration frequency and vibration magnitude in Experiment 2. ( $\mathrm{Y}$ error bars $=95 \%$ C.I. .

Figure 4.2.8 Force-recall error as a function of vibration magnitude and exertion type (grip or push) across all conditions of Experiment 2.

Figure 4.2.9 Force-recall error as a function of vibration magnitude and exertion type (grip or push) in Experiment 2. (Y error bars $=95 \%$ C.I.).

Figure 4.2.10 Force-recall error for grip and push plotted against relative force level for Experiment 2

Figure 4.2.11 Force-recall error for grip and push plotted against relative force level for Experiment 2. (Y error bars $=95 \%$ C.I.)

Figure 4.2.12 Interaction between gender and exertion type (grip or push) across all conditions of Experiment 2.

Figure 4.2.13 Interaction between gender and exertion type (grip or push) in Experiment 2. (Y error bars $=95 \%$ C.I.)

Figure 4.3.1 Distribution of recalled forces for (a) grip and (b) push by target force across all vibration exposure conditions of both experiments.

Figure 4.3.2 Grip and push force-recall error means plotted against vibration frequency across all conditions of the two experiments.

Figure 4.3.3 Grip and push force-recall error means plotted against vibration frequency for both experiments. ( $\mathrm{Y}$ error bars $=95 \%$ C.I.) 
Figure 5.1.1 Force-recall error expressed as a percentage of target force for grip and push plotted against relative force level for Experiment 1 ..

Figure 5.1.2 Force-recall error expressed as a percentage of target force for grip and push plotted against relative force level for Experiment 104 


\section{LIST OF TABLES}

Table 2.3.1 Dominant vibration frequencies of some common powered hand tools (adapted from Griffin 1997).

Table 3.4.1 Single-axis sinusoidal vibration exposures used in Experiment 1..........................43

Table 3.5.1 Single-axis sinusoidal vibration used during Experiment 2 2...................................48

Table 4.1.1 Descriptors of the twelve male participants of Experiment 1................................53

Table 4.1.2 Recalled grip and push forces (in Newtons) across all vibration exposure conditions of Experiment 1......................................................................................5

Table 4.1.3 ANOVA of grip and push force-recall error in the Experiment 1.........................56

Table 4.1.4 Sensitivity of Experiment 1 for detecting force-recall error differences due to vibration frequency effects at three levels of type II error probability $(\beta)$.

Table 4.1.5 Sensitivity of Experiment 1 for detecting force-recall error differences due to the vibration frequency by force level interaction effects at three levels of type II error probability $(\beta)$.

Table 4.1.6 Force-recall error (in Newtons) at each vibration frequency in Experiment 1.......59

Table 4.1.7 Tukey's HSD pair-wise recall error mean comparisons for vibration frequency in Experiment 1.

Table 4.1.8 Force-recall error (in Newtons) at each level of hand-handle coupling force in Experiment 1.

Table 4.1.9 Force-recall error (in Newtons) for each test session in Experiment 1.

Table 4.1.10 Test-retest correlations for recalled grip and push forces for each subject across all conditions for Experiment 1

Table 4.1.11 Grip and push force-recall error means (in Newtons) across all conditions of Experiment 1.

Table 4.1.12 Force-recall error means (in Newtons) at each rest period duration evaluated in Experiment 1 .

Table 4.1.13 Force-recall error means (in Newtons) for each exertion type and rest period duration evaluated in Experiment 1

Table 4.2.1 Descriptors of the twenty participants of Experiment 2.

Table 4.2.2 Distribution of recalled grip and push forces (in Newtons) across all vibration exposure conditions of Experiment 2 
Table 4.2.3 ANOVA of grip and push force-recall error in Experiment 2.

Table 4.2.4 Sensitivity of Experiment 2 for detecting force-recall error differences due to vibration frequency effects at three levels of type II error probability $(\beta)$. .74

Table 4.2.5 Sensitivity of Experiment 2 for detecting force-recall error differences due to the vibration frequency by force level interaction effects at three levels of type II error probability $(\beta)$. .75

Table 4.2.6 Force-recall error means (in Newtons) at each vibration frequency in Experiment 2

Table 4.2.7 Tukey's HSD pair-wise comparisons for vibration frequency in Experiment 2 .79

Table 4.2.8 Grip and push force-recall error means (in Newtons) in Experiment 2. 80

Table 4.2.9 Means (in Newtons) for force-recall error at the zero vibration condition and at each level of vibration magnitude used in Experiment 2.

Table 4.2.10 Means (in Newtons) of force-recall error at each level of vibration frequency and magnitude combination used in Experiment 2.

Table 4.2.11 Means (in Newtons) for grip and push force-recall error at each level of vibration magnitude exposure in Experiment 2.

Table 4.2.12 Means (in Newtons) for force-recall error at each level of applied force in Experiment 2 .

Table 4.2.13 Means (in Newtons) for force-recall error for each test session in Experiment 2 .

Table 4.2.14 Test-retest correlations for recalled grip and push forces for each subject across all conditions for Experiment 2.

Table 4.2.15 Means (in Newtons) for force-recall error for each gender in Experiment 2....... 89

Table 4.2.16 Means (in Newtons) for force-recall error for each gender and exertion type (grip or push) across all conditions of Experiment 2.

Table 4.3.1 Grip and push forces (in Newtons) applied during the force memorization and recall periods of Experiments 1 and 2 .

Table 4.3.2 Distribution of recalled grip and push forces (in Newtons) across all vibration exposure conditions of both experiments.

Table 4.3.3 Force recall-error means (in Newtons) at each vibration frequency evaluated in the two experiments. 


\section{H A P T E R 1: I N T R O D U C T I O N}

\subsection{Background}

The operation of percussive tools such as rock drills, chipping hammers, and jack hammers frequently requires forceful and repeated push and grip actions to control the tools and to achieve the desired productivity. These tools are also known to generate high magnitudes of hand-transmitted vibration which is an additional stressor. A tight hand-tool coupling not only imposes high stresses on the anatomical structure of the hand-arm system and impedes peripheral circulation, but it also increases vibration transmissibility to the hand and arm (Carlsöö and Mayr 1974, Brammer 1982, Hartung et al. 1993, Riedel 1995). It has been demonstrated in the scientific literature that workers exposed to hand-transmitted vibration produced by powered hand tools and other vibration sources are at risk of developing circulatory, neurological, or musculoskeletal disorders (Starck et al. 1990, Kihlberg and Hagberg 1997, NIOSH 1997, Bovenzi 1998, Griffin and Bovenzi 2001). These disorders have been included in a group known collectively as hand-arm vibration syndrome (HAVS) (Gemne and Taylor 1983).

The need for assessing HAVS risks associated with the operation of vibrating tools is well established. It is also well known that the amount vibration actually transmitted to the handarm system is dependent on the coupling forces at the hand-tool interface (Taylor and Brammer 1982, Radwin et al. 1987, Hartung et al. 1993, Riedel 1995, Kaulbars 1996, Wasserman 1998). The use of vibrating tools in combination with forceful and repetitive hand motions may also result in a greater incidence of other forms of cumulative trauma 
disorders (CTDs) such as carpal tunnel syndrome (CTS) (Cannon et al. 1981, Silverstein et al. 1987) and hand/wrist tendonitis.

The International Organization for Standardization (ISO) has issued an international standard for the assessment of exposure to hand-transmitted vibration, ISO-5349-1 (ISO 2001). This standard recommends the establishment of a dose-response relationship for evaluating risk in terms of exposure duration, frequency of vibration, and frequency magnitude. The standard calls for the measurement of acceleration at the surface where vibration energy enters the body. Typically, this means that the acceleration is measured by fastening an accelerometer to the hand grip of the vibrating structure.

With regard to hand coupling forces, ISO-5349-1 states:

Although characterization of the vibration exposure currently uses the acceleration of the surface in contact with the hand as the primary quantity, it is reasonable to assume that the biological effects might depend, to a large extent, on the energy transmitted. This energy depends on the coupling of the hand-arm system to the vibration source and, consequently, on the grip pressure applied and the magnitude and direction of the static force. Measurement of the energy transmitted to the hand and of the tool application force is feasible and desirable for research purposes and for future application to special tools, but is not yet proposed in this International Standard. 
Thus, ISO recognizes that hand coupling forces influence the vibration energy transmitted to the hand, but they fail to offer specific guidance on how to characterize those forces.

Recognizing strong contributions of hand forces, correction factors have been proposed for improved application of the ISO standard (Riedel 1995, Kaulbars 1996). Nevertheless, these correction factors yield poor estimates of the hand contact forces (Welcome et al. 2004). The need to quantify the forces acting at the hand-handle interface has been further emphasized in a recent ISO draft international standard (ISO 2005). While this document attempts to establish definitions and standardize terminology, it contains no guideline or methodology for hand force measurement. Likewise, neither the current NIOSH recommendations (NIOSH 1989) nor the American National Standards Institute (ANSI) standard (ANSI 1986) for assessing hand-arm vibration exposure offer methodologies for hand force quantification. Because the interaction between hand and object is so critical, comprehensive risk assessments of HAVS, CTS, and other hand-arm musculoskeletal disorders must include an evaluation of hand forces, postures, and motions. Unfortunately, there has been no consensus as to how to best measure those forces. An understanding of the nature of hand forces applied to tools is essential for developing appropriate strategies and working procedures geared to minimize stress and transmitted vibration.

Hand forces can be measured using various techniques, although a number of these techniques are limited to laboratory applications. The choice of measurement technique, however, depends on the nature of the observed task and practical considerations such as required accuracy and the availability of equipment and expertise. A considerable number of studies on hand force measurement techniques have been published (Radwin et al. 1991, 
Gurram et al. 1995, Kaulbars 1996, Radwin and Yen 1999, Chadwick and Nicol 2001, McGorry 2001, Wasserman et al. 2001). While hand forces can be effectively and accurately measured with dynamometers and handgrip and pinch strength meters, it remains a formidable task to quantify the hand force applied to tools in the workplace.

Several methods have been used to measure the hand coupling forces on tool handles. One way is to instrument the tool handle using strain gauges or force transducers (Radwin and Yen 1999, McGorry 2001). However, the application of instrumentation to tool handles may alter the hand postures and contact areas on the handle surface such that the measured forces may not offer an accurate account of the coupling forces actually utilized by workers. Some manufacturers have largely overcome this confounding factor by developing wafer-thin, flexible force sensors that can be wrapped around the tool handle or incorporated into a work glove (Gurram et al. 1995, Wasserman et al. 2001, Welcome et al. 2004). Unfortunately, such instrumentation installations can be expensive and too fragile for many workplace environments. Therefore, alternative methods for coupling force quantification need to be developed.

One promising force measurement alternative involves a psychophysical method of force recall. This type of measurement is carried out by asking a test subject to reproduce his/her hand force with a similar type of hand-handle coupling action (e.g. push, power grip, or pinch grip) on a dynamometer, grip strength meter, or pinching strength meter. Several investigators (Lowe 1995, Wiktorin et al. 1996, Bao and Silverstein 2005) have studied the reliability and accuracy of this method. These studies demonstrated that the subjects could reproduce familiar forces reasonably well, and the force-recall method could be considered 
reliable within certain force ranges. However, these studies were performed with manual tasks or simulated labor in the absence of hand-transmitted vibration exposure. The accuracy and reliability of the force-recall method under exposure to hand-transmitted vibration have not been reported in the scientific literature.

The difficulties encountered when quantifying hand forces on tool handles are also problematic in the evaluation and development of sports equipment, motor vehicles and mobile equipment, and any other mechanisms that involve hand forces. In short, there are many applications outside of occupational safety and health where a standardized force-recall method would be beneficial. While this dissertation focuses on hand force quantifications as elements of work task evaluations, the principles involved with force recall can be applied to a myriad of human activities.

\subsection{Objectives and hypotheses}

The overall objective of the research is to contribute towards the base of knowledge necessary for the development of psychophysical hand force quantification methods. These hand force measuring techniques can be integrated into comprehensive risk assessments for HAVS, CTS, and other musculoskeletal disorders of the upper extremities. Without a thorough understanding of the factors that can influence force recall accuracy, these psychophysical techniques cannot be optimized. Once these techniques are refined, force recall methods can be used to quantify hand forces associated with the operation of vibrating tools and may be incorporated into risk assessment strategies. 
The major objective of this research is to provide information regarding specific factors that may influence the accuracy of force-recall procedures that may be used to estimate grip and push forces applied to vibrating tools. There are three primary specific aims of this research. They are:

(i) to examine the effects of vibration frequency on a subject's ability to recall and duplicate target grip and push forces applied to an instrumented handle;

(ii) to examine the effects of vibration magnitude on a subject's ability to recall and duplicate target grip and push forces applied to an instrumented handle; and

(iii) to examine the effects of grip and push force magnitude on a subject's ability to recall and duplicate target grip and push forces applied to an instrumented handle.

Two experiments were conducted to address these specific aims. For the first specific aim, force-recall accuracy was evaluated at eight levels of vibration frequency $(0 \mathrm{~Hz}, 12.5 \mathrm{~Hz}, 16$ $\mathrm{Hz}, 31.5 \mathrm{~Hz}, 40 \mathrm{~Hz}, 63 \mathrm{~Hz}, 125 \mathrm{~Hz}$, and $250 \mathrm{~Hz}$ ). In the first experiment, five levels of vibration were evaluated ( $0 \mathrm{~Hz}, 12.5 \mathrm{~Hz}, 40 \mathrm{~Hz}, 125 \mathrm{~Hz}$, and $250 \mathrm{~Hz})$. The null and alternative hypotheses for this experiment were:

$$
\begin{aligned}
& H_{0}: \mu_{0}=\mu_{12.5}=\mu_{40}=\mu_{125}=\mu_{250} \\
& H_{a}: \text { at least one inequality }
\end{aligned}
$$


In the second experiment, five levels of vibration were evaluated $(0 \mathrm{~Hz}, 16 \mathrm{~Hz}, 31.5 \mathrm{~Hz}, 63$ $\mathrm{Hz}$, and $125 \mathrm{~Hz}$ ). The null and alternative hypotheses for this experiment were:

$$
\begin{aligned}
& H_{0}: \mu_{0}=\mu_{16}=\mu_{31.5}=\mu_{63}=\mu_{125} \\
& H_{a}: \text { at least one inequality }
\end{aligned}
$$

For the second specific aim, force-recall accuracy was tested at two levels of vibration magnitude. The levels of vibration magnitude were the 4 -8-hour and $<0.5$-hour frequencyspecific exposure limits found in ANSI S3.34 (1986). This specific aim was primarily addressed in the second experiment. The null and alternative hypotheses for this specific aim were:

$$
\begin{aligned}
& H_{0}: \mu_{H I}=\mu_{L O} \\
& H_{a}: \mu_{H I} \neq \mu_{L O}
\end{aligned}
$$

For the third specific aim, force-recall accuracy was evaluated at three levels of hand force ( $15 \mathrm{~N}$ grip/25N push, $30 \mathrm{~N}$ grip/50N push, and $45 \mathrm{~N}$ grip/75N push). This factor was evaluated in both experiments. The null and alternative hypotheses for this specific aim were:

$$
\begin{aligned}
& H_{0}: \mu_{L O}=\mu_{M E D}=\mu_{H I} \\
& H_{a}: \text { at least one inequality }
\end{aligned}
$$




\subsection{Benefits of the research}

In the United States alone, more than one million workers are exposed to hand-transmitted vibration (Griffin 1990). As stated earlier, extensive use of vibrating hand tools has been related to an array of potentially disabling disorders collectively labeled as HAVS. The amount of vibration actually transmitted to the hand-arm system depends on the coupling forces at the tool handle. Therefore, quantifying the hand forces at the tool handle should be an essential component of a comprehensive HAVS risk assessment for the protection of exposed workers.

Unfortunately, current standards on hand-transmitted vibration exposure, while recognizing the importance of characterizing hand coupling forces, do not include quantitative assessments of these forces. This is mainly due to the lack of practical methods for acquiring hand force data. Observer's estimations of hand force magnitudes are often inaccurate. Instrumentation can be expensive and ill-suited for field assessments. The psychophysical force-recall technique may prove to be a viable alternative for quantifying hand forces, but various factors may influence a subject's ability to accurately reproduce those forces. Therefore, the effects of those factors on force-recall accuracy need to be explored.

This research is a part of the ongoing systematical development of new methodologies for quantifying hand forces being conducted at the National Institute for Occupational Safety and Health (NIOSH) in Morgantown, West Virginia. The results derived from these experiments will be used by NIOSH investigators in their subsequent studies as well as by investigators outside of NIOSH. 
The specific potential benefits of this research are as follows:

a. The study will develop new laboratory methods for the study of potentially influential factors in psychophysical force-recall trials.

b. $\quad$ This investigation will provide knowledge of the association between vibration frequency and the accuracy of the force-recall technique.

c. This investigation will provide knowledge of the association between vibration magnitude and the accuracy of the force-recall technique.

d. This investigation will provide knowledge of the association between hand force level and the accuracy of the force-recall technique.

e. This investigation will provide knowledge of any significant interactions between the three main effects.

f. $\quad$ This investigation will explore the accuracy and reliability of this forcerecall technique.

g. The results of the study are expected to provide the basis for improved methodologies for accurately and reliably estimating hand forces applied to tool handles in field and laboratory environments.

h. The results of the study are expected to contribute considerably to standardized risk assessments for HAVS, CTDs, and other upper extremity disorders associated with hand forces. 


\section{H A P T E R 2: R E V I E W O F L I T E R A T U R E}

\subsection{Psychophysics in the study of work}

There are psychophysical sensations associated with the production of a static force or the motion of a limb. Several early researchers have shown that the magnitudes of these sensations are functions of certain stimuli and can be quantified (Stevens 1957, Stevens and Mack 1959, Borg 1962, Eisler 1962). It has also been shown that humans have the ability to develop an awareness of these sensations and to rate, categorize, or replicate their efforts. Knowledge of these reliable behaviors has led to the successful development of many techniques for analyzing work tasks. Presented below are summaries of a few examples of psychophysical techniques used in work analyses.

One set of subjective ratings, commonly referred to as Rated Perceived Exertion (RPE), has been used extensively in the evaluation of various work tasks (Borg 1962, 1982). Borg's popular rating system has proven to provide relatively consistent assessments of worker discomfort and/or effort. The Borg scale ranges from 0 to 10 , with ' 0 ' representing no discomfort or effort, and '10' equaling extreme discomfort or effort.

Some subjects tend to rate exertion or discomfort at the low end of the scale while others consistently deviate towards the high end. While each individual develops a personal assessment criteria, studies have shown that a subject's RPE is highly repeatable for many varied work postures (Wangenheim et al. 1986). In the Wangenheim et al. study (1986), 31 healthy university students used the Borg scale to rate their perceived exertion while 
maintaining numerous postures. The set of 78 postures included head and neck postures, shoulder-arm postures, hand-wrist postures, back-trunk postures, hip postures, knee postures, and foot-ankle postures. In the experiment, the subjects would assume a target posture, hold the posture, and report their perceived exertion level after 15, 30, and 45 seconds. While some subjects were consistently "low raters" and some were consistently "high raters," the rating patterns were relatively uniform. The results of the study showed that the RPE scale could be used successfully to evaluate and compare different body postures.

Psychophysical techniques have been accepted as primary criteria for determining safe lifting limitations (Garg et al. 1980), and many studies have been conducted to evaluate psychophysical approaches to materials handling task assessment. For example, Ciriello et al. (1990) asserted that psychophysical methods are appropriate for determining maximum acceptable weights and forces associated with materials handling tasks. In their study, 22 industrial workers (12 female and 10 male) performed 18 variations of pushing, pulling, lifting, and carrying tasks. The subjects were asked to select the amount of weight to lift, lower, and carry, or to adjust their pushing and pulling forces to the maximum level that they felt they could sustain for 8 hours without "straining themselves or without becoming unusually tired, weakened, overheated, or out of breath." The researchers found that, by and large, the psychophysically-determined weights/forces chosen during the first 40 minutes remained reliably consistent throughout each 4-hour session. The authors concluded that their psychophysical method for determining maximum acceptable weights and forces was appropriate for similar tasks to be performed over an 8-hour shift. 
Several other researchers have used psychophysical approaches such as perceived effort ratings as bases for lifting capacity models (Snook 1978, Garg et al. 1980, Pytel and Kamon 1981, Karwowski et al. 1984). In the Snook publication (1978), the results of seven experiments were summarized for use in the design of manual material handling tasks. The same basic psychophysical technique was used in each of the studies. Briefly, experienced industrial workers performed material handling tasks such as lifting, lowering, and carrying tote boxes or pushing or pulling loads (as simulated on a treadmill). The subjects were able to increase or decrease their loads in order to obtain what they believed to be their maximum sustainable loads. The subjects were not provided with any information regarding the initial or adjusted weights. The boxes were constructed with false bottoms in order to eliminate visual cues. The subjects varied the treadmill push/pull force levels by turning a knob that throttled the amount of electric current flowing into a magnetic clutch. The current-adjustment mechanism provided no visual cues. The subjects were instructed to work as hard as they could without becoming unusually tired. They were provided with five days of training so that they could become accustomed to the psychophysical task of monitoring their sensory perception and making the weight and force level adjustments. Snook found that the results from each study were normally distributed. He used the results to predict the percentage of the industrial population that could be expected to perform similar tasks without excessive fatigue. The means and standard deviations were used to determine the maximum weights and forces that would be acceptable to $10,25,50,75$, and $90 \%$ of the target population.

The Garg et al. study (1980) was designed to compare psychophysically-determined lifting capacities with isometric test results. The researchers used nine healthy college students with 
some background in occupational biomechanics, and each had some familiarity with manual material handling operations. A week prior to data collection, the subjects attended two, 3-hour training sessions involving the static and dynamic lifting strength measurements. For each task, the subjects were instructed to add or subtract weight from a tote box until they reached what they felt was the maximum acceptable load that they could lift comfortably once every 15 minutes. The subjects performed a similar task that involved a static load. The authors concluded that, if carefully designed, a static strength test could predict a worker's dynamic lifting capacity as accurately as a psychophysical technique.

Legg and Myles (1981) concluded that psychophysical techniques could be used to reliably and repeatedly identify loads that military personnel could lift repetitively over an 8-hour period without metabolic, cardiovascular, or subjective evidence of fatigue. For this study, the two researchers used 10 healthy male soldiers who had all previously participated in repetitive lifting studies. Using the Snook (1978) method, each soldier selected the maximum load that he thought he could lift and lower between the floor and a bench positioned at a level of $40 \%$ of his stature. They were told that the lifting rate was to be set at five lifts every two minutes over the course of an 8-hour work shift. Twice per day for a five-day period, each subject performed a 20-minute maximal acceptable load (MAL) determination. At no time during the study were the subjects aware of the magnitudes of their MALs. The average of the ten sessions was used as the subject's MAL. Following the five-day MAL determination, each subject lifted and lowered their MAL five times during each two-minute period over the course of an 8-hour shift. Expired gas was collected, and heart rates were recorded every 30 minutes, and oxygen consumption and energy expenditure were calculated. The subjects were also 
asked to estimate their Rated Perceived Exertion on a 10-point Borg scale as well as on a $100 \mathrm{~mm}$ line with its left and right anchors labeled "very, very light" and "very, very hard," respectively. In addition to the RPE, the subjects also were instructed to indicate their estimations of "sustainable duration" on $100 \mathrm{~mm}$ lines. Sustainable duration was defined as how long they thought they could continue working at the same pace and with the same load without straining or becoming unusually tired. The analysis of the data showed that while the subjective ratings of fatigue and the Borg RPE ratings significantly increased during the morning sessions and tended to increase in the afternoon, energy expenditure and heart rate did not change significantly during the 8-hour work shift. None of the subjects displayed metabolic or cardiovascular evidence of fatigue during their work shifts. Furthermore, none of the soldiers rated the their workloads as unduly fatiguing, and none reported aches or pains as a result of the work. Thus, the researchers concluded that this psychophysical technique for MAL determination can be used to identify workloads that soldiers can sustain for an 8-hour work shift. The authors also noted that the soldiers underestimated the duration for which they could sustain their work intensity. The average estimation of how long they could continue lifting and lowering their MALs was between six and seven hours. However, all of the subjects were able to complete their entire 8-hour shifts without any evidence of cardiovascular or metabolic fatigue.

It should be noted that work orientation and posture have been shown to significantly affect subjective fatigue and discomfort ratings (Wiker et al. 1990, Ulin et al. 1993, Jung and Hallbeck 2002). In a Schoenmarklin and Marras study (1989), eight healthy male subjects (all hammer novices) performed two hammering tasks; one task had the target spike in a wall- 
mounted position, while the other task had the spike in a bench-mounted apparatus.

Immediately following each three-minute hammering task, the subjects were asked to rate their discomfort on an ordinal scale from 1 to 9 . The subjects gave ratings for 10 body segments: hand, wrist, forearm, elbow, arm, shoulder, neck, upper back, middle back, and lower back. For nearly every body segment, the differences in the discomfort ratings were significantly different for the various postures.

The Ulin et al. study (1990) was designed to determine if there were differences in worker preference for a screwing task performed at seven different heights and to compare three psychophysical scales. Thirty-six subjects (university students) participated in the study. Each subject was asked to drive No. 6 hex-head sheet metal screws into a sheet of 18-gauge perforated sheet metal. Using an air-powered pistol-shaped screw gun, each subject drove 25 screws into the perforated metal at seven vertical locations. The subjects were paced by an electronic beeper to maintain a 5 -second rest period between screws. At each vertical location, the subjects were asked to rate their comfort level and their assessment of task difficulty. Three scales were used for the subjective ratings; the Borg (1982) 10-point scale and two visual analog scales. The Borg scale ranged from 0 (nothing at all) to 10 (very, very hard). The two analog scales each consisted of a horizontal bar bounded by vertical lines. One horizontal bar was labeled "COMFORT" and the second bar was labeled "IMAGINE." The extremes of the Comfort bar were labeled "very uncomfortable work" and "very comfortable work," respectively. The anchors of the Imagine bar were marked "easiest imaginable work" and "hardest imaginable work." The subjects were asked to perform task assessments with each of the three psychophysical scales after driving screws at each of the seven levels. The 
subjects were asked to select a number from the Borg scale and to draw a line on each of the two analog scales to represent their ratings. Statistical analyses revealed that there was a reliable difference between the ratings at the seven levels. Correlation analyses showed that the three rating scales were significantly correlated. It is interesting to note that when surveyed, 27 of the 36 subjects stated that they preferred to use the Borg scale over the two analog scales, but the results indicated that any of the three scales could be used with reliable results.

In a subsequent screw driving experiment (Ulin et al. 1993), 18 industrial workers (9 males, 9 females) assessed their perceived exertion level with the 10-point Borg scale (Borg 1970, 1990) after driving screws into perforated sheet metal at different orientations with air-powered tools with various masses. The subjects also rated their level of discomfort with a visual analog scale similar to one used in the previously-described Ulin et al. study (1990). The object of the study was to examine the effects of tool mass and work posture on perceived exertion and discomfort. Each subject performed the screwing task with three tools at each of five orientations. The subjects also completed a body part discomfort survey in which they shaded areas of a diagram depicting the human body. The subjects shaded the areas where they felt discomfort, and then they rated the level of discomfort by marking a $10 \mathrm{~cm}$ line with anchors labeled "No Discomfort at all" and "Worst Imaginable Discomfort." The researchers found that tool mass and work location were each significant factors in determining perceived exertion and level of discomfort. The ratings of perceived exertion increased with tool mass in all five work orientations. The discomfort ratings also varied with posture. The authors concluded that the results of their study supported the notion that workstation design should 
follow psychophysical research, and they hypothesized that such workstation designs would reduce the occurrence of work-related disorders.

\subsection{Perception and recall of movement and manual forces}

It has been shown that humans have the ability to memorize and replicate the forces and movements associated with work tasks (Hammarskjöld et al. 1989, 1990, Lowe 1995, Wiktorin et al. 1996, Bao and Silverstein 2005). Using the same principles involved with perceived force and perceived exertion ratings, subjects can reproduce familiar forces and movements based on musculoskeletal, skin, and subcutaneous sensory feedback and position sense. While workers have not shown the ability to accurately quantify their exerted forces (Wiktorin et al. 1996), subjects have shown remarkable ability to correctly rank weights and forces (Wang et al. 1991, Karwowski et al. 1992, Kumar and Simmonds 1994, Kumar et al. 1994, Wiktorin et al. 1996).

An experiment in the Wiktorin et al. (1996) study tested subjects' abilities to recall and duplicate forces involved in day-to-day work tasks commonly performed by the subjects. The subjects demonstrated remarkable reproducibility in generating a wide range of sub-maximal forces in a wide variety of postures. Both dynamic lifting and isometric tasks were simulated with repeatable results. The authors stated, "It seems reasonable that the neuro-muscular system 'remembers' the manual forces exerted and can therefore reproduce them with high precision.” 
One recent study (Bao and Silverstein 2005) found that subjects can consistently reproduce a wide range of pinch and grip forces on a handle dynamometer, and that subjects can accurately match grasp and pinch forces required to perform routine tasks such as closing a staple remover, lifting a ream of paper, cutting wire with pliers, holding a dumbbell, squeezing a soft drink can, and opening a battery jumper cable clamp.

The Lowe (1995) study showed that subjects can reproduce four target forces as well as they can match two target forces. The Lowe study focused on grip forces measured on a handle dynamometer. Lowe's subjects were asked to match target grip forces that were based on percentages of each subject's maximum voluntary contraction (MVC). Lowe divided his subjects into three groups. The individuals in the first group attempted to "memorize" and match targets that were $20 \%$ and $35 \%$ of their MVC. The subjects in the second group matched $50 \%$ and $65 \%$ MVC targets. The third group's targets were $20 \%, 35 \%, 50 \%$, and $65 \%$ MVC. The results indicated that the number of target forces to be matched had no significant effect. That is to say, the subjects who were required to memorize four targets were no less accurate than the subjects who needed to recall only two reference forces. The Lowe study results suggest that humans have a the capacity to memorize and reproduce multiple forces associated with a complex task.

While some of the reported force-matching or recall studies focused on pinch and grasping forces, others have looked at other motions and forces. Watson et al. (1984) found that subjects could reliably match forces required to move spring loads attached to the forearm with bending at the elbow only. One study (Carlsöö 1986) found that subjects could reproduce 
certain static forces (two-hand lift, thigh lift, and foot pedal operation) several times consecutively with variations of just under 10 percent. Carlsöö found that the subjects were equally precise when matching familiar task forces as they were when matching the forces of unfamiliar tasks.

While the search for the precise mechanisms involved in force perception and force recall remains a formidable research task, many studies have provided evidence to support the notion that the sensorineural information generated during the production of muscle forces comprises signals from the central nervous system as well as from various sensory units (McCloskey 1981, Nowak et al. 2003). Moreover, perceived effort and force recall appear to be functions with both efferent (central) and afferent (peripheral) components (Gandevia et al. 1990, Miall et al. 2000).

The group of efferent signals consists of the communications between the central nervous system and the muscles. The efferent signals control which muscles become involved with a specific action, as well as the magnitudes of muscle tension or motor forces imparted. Studies have indicated that a copy of the motor cortex outflow is simultaneously transmitted to the sensory cortex in a feed-forward mechanism. This mechanism has been referred to as “corollary discharge" or "efference copy" (McCloskey 1981). Several researchers have concluded that these efferent communications form the foundation for a subject's perception of how much an object weighs or the amount of effort required to complete a motor task (Lacourse and Morris 1991, Burgess and Jones 1997). Other researchers have suggested that efferent signals can be stored and retrieved from memory. 
Lafargue et al. (2003) stated it this way:

Perceived heaviness and perceived force primarily result from the degree of efferent activity in the motor system. The physiological explanation generally accepted is the following: when the brain initiates a motor command, it keeps a record of this information through corollary discharge or efferent copy of the command. The record can be used itself as a measure of effort.

While central signals seem to dominate human force perception, it is nearly universally accepted that peripheral components also play a significant role in force production perception (Goodwin et al. 1972, Lafargue et al. 2003). It has been estimated that the afferent contributions account for about 30 percent of the overall sensation of intramuscular tension (Cafarelli and Bigland-Ritchie 1979). Therefore, the effects of vibration on afferent signal production should not be ignored.

The afferent information can be divided into two sets: muscle afferents and cutaneous afferents. The muscle afferent information is produced by peripheral receptors in the muscles, joints, and tendons that provide feedback to the sensory cortex. These transmissions contain information regarding muscle extension, contraction, and tension along with joint position. The cutaneous afferent signals from the hand carry information regarding textures, sizes, shapes, and pressures imposed on the skin and subcutaneous tissues. Most subjects have the ability to distinguish the characteristics of each of these afferent mechanisms, but a change in 
one can alter the perception of another (Henningsen et al. 1995b). Subjects can use the afferent information to estimate the weights of objects or to make judgments about grip, pinch, or push forces required to move an object or complete a task. A recent study indicates that muscle afferents are more influential than cutaneous afferent feedback as regards a subject's ability to maintain isometric forces (Ebied et al. 2004).

McCloskey (1974) concluded that subjects can accurately estimate the weights of grasped objects using either afferent or efferent signals, but not in the absence of both. Similarly, the results of two Henningsen et al. studies (1995b, 1995a) suggested that tactile afferent signals from the nerves of the fingertips were important for subjects' determinations of the magnitudes of voluntarily exerted forces. Furthermore, their studies indicated that finger force perception depends more on the extent to which the fingertip skin is indented as opposed to the local pressures exerted at the skin. The researchers concluded that the forces exerted by each finger depends on the subject's hand dominance as well as the shape of the contact surface. Furthermore, the authors stated that the findings "imply that the perception of force is dependent on several factors such as information from periphery and asymmetries inherent in the motor system."

Like perceived effort, position sense has been described as a sensorimotor utility with both afferent and efferent components (Goodwin et al. 1972, Heide and Molbech 1973, Feldman and Latash 1982, Matthews 1982). Feldman and Latash (1982) proposed that without afferent signals, efferent information loses its meaning. Thus, they concluded that in the absence of afferent signals, subjects are unable to perceive movement and cannot accurately estimate 
changes in the position of a joint. Heide and Molbech (1973) affirmed that during attempts to match a reference position of a limb, efferent and afferent information are combined by the central nervous system to account for a person's memory of the limb's reference position. When attempting to reproduce the reference position, the subject tries to duplicate the motor and sensory information from memory.

\subsection{Potential effects of vibration on force recall}

\subsubsection{Vibration frequencies of concern}

While workers using vibratory tools may encounter a wide range of vibration frequencies, nearly all vibrating tools exhibit a dominant frequency that falls below $250 \mathrm{~Hz}$. Therefore, a study of vibration effects on force recall accuracy should focus on that frequency range. Table 2.3.1 contains dominant vibration frequencies of several common powered hand tools (adapted from Griffin 1997). 
Table 2.3.1 Dominant vibration frequencies of some common powered hand tools (adapted from Griffin 1997).

\begin{tabular}{lc}
\hline \multicolumn{1}{c}{ Tool } & $\begin{array}{c}\text { Dominant vibration } \\
\text { frequency }(\mathrm{Hz})\end{array}$ \\
\hline Road tamper (gasoline) & 12.5 \\
Road breaker (pneumatic) & 16 \\
Impact wrench & 31.5 \\
Nutrunner & 31.5 \\
Rock drill (pneumatic) & 40 \\
Metal drill & 50 \\
Needle gun & 50 \\
Chipping hammer (pneumatic) & 63 \\
Riveting gun & 63 \\
Orbital sander & 100 \\
Straight grinder (5", pneumatic) & 100 \\
Vertical grinder (7", pneumatic) & 100 \\
Angle grinder (9", electric) & 100 \\
Standard chainsaw & 125 \\
Anti-vibration chainsaw & 200 \\
Etching pen & 200 \\
Rotary file (pneumatic) & 200 \\
\hline
\end{tabular}

\subsubsection{Vibration effects on efferent and afferent signals}

The effects of vibration on force-recall ability are virtually unknown, but there is considerable evidence to suggest that vibration may effect both the efferent and the afferent components. Muscle or tendon vibration may induce motor sensory illusions such as a perceived increase in muscle length or false rotational movement (Goodwin et al. 1972, Feldman and Latash 1982, McCloskey et al. 1983). These or other vibration-induced illusions may lead to force-recall errors. Miall et al. (2000) concluded that vibration may interfere with a subject's sense of limb position and motion, and thus, reduce accuracy of perceived effort. In their study, five subjects 
exposed to localized vibration at a frequency of $100 \mathrm{~Hz}$ were unable to accurately judge substantial weight changes of a water-filled container.

Another potential contributor to force-recall errors is the disruption of the skin and subcutaneous afferents. There are several nerve fibers in the skin and sub-cutaneous tissues responsible for mechanoreceptive afferent flow. Briefly, the units are classified as either fast adapting (FA) or slow adapting (SA) and are divided into two types according to size and shape (Johansson and Vallbo 1983). Group I units are smaller and have sharp borders, while Group II units are larger and have relatively obscure borders. All of these units are sensitive to vibration in varying degrees. Of these units, the FA II units (Pacinian corpuscles and GolgiMazzoni bodies) are the most sensitive to vibration (Lundström and Johansson 1986). Further, Verrillo (1975) identified the Pacinian corpuscle as the primary receptor for vibration perception. The FA II units are most sensitive when exposed to vibrations between 100 and $300 \mathrm{~Hz}$ (Johansson and Vallbo 1983). Therefore, it is reasonable to presume that vibrationinduced disturbances to the FA II unit outflow may play a role in force recall accuracy, particularly with vibration exposures between $100 \mathrm{~Hz}$ and $300 \mathrm{~Hz}$.

\subsubsection{Tonic vibration reflex}

Another possible influence on force-recall accuracy is a phenomenon known as the tonic vibration reflex (TVR). Several researchers in the 1960s discovered that when a moderately active muscle was stimulated by vibration, the activity in that muscle increased while the activity in the antagonist muscles decreased (Hagbarth and Eklund 1965, de Gail et al. 1966). 
However, in most TVR experiments, the reflex action is initiated by directly applying vibration to the muscle tendon. In fact, in their early study of this reflex phenomenon, Hagbarth and Eklund (1965) observed that the reflex action was not normally induced unless the vibration was directly applied to the muscle tendons, and that any reflex action was limited to the muscles whose tendons were stimulated. Thus, it is unlikely that a voluntary power grip or palm push motor function could be affected by a reflex action initiated through handtransmitted vibration. Furthermore, early TVR studies showed that even when the vibration stimulus was directly applied to the tendon, subjects could counteract or prevent the reflex actions, especially if there was visual feedback of limb position or muscle force (Hagbarth and Eklund 1965, de Gail et al. 1966).

\subsubsection{Resonance of the hand and arm systems}

The human hand and arm can be regarded as a biomechanical system consisting of various

rigid, springy, and viscous tissues. The hand-arm system and its sub-systems respond differently to various vibration exposures and with different levels of hand-tool coupling stiffness (i.e., grip and push force) for any given vibration frequency. The system's response to vibration can affect the motions and stresses of the various tissues involved in force production and perception. Hence, the biodynamic response of the hand-arm system to vibration may affect perceived effort and force recall accuracy.

Common terms used to describe these biodynamic responses to vibration include dissipated power, vibration transmissibility (expressed as a transfer function), and apparent mass. However, the biodynamic response has usually been expressed in terms of mechanical 
impedance (Lundström 1984, ISO 1998, Dong et al. 2004b). A high mechanical impedance value indicates that the system is more responsive to vibration stimuli. Mechanical systems, including the hand-arm system, exhibit particularly high impedance values at certain vibration frequencies. The frequency at which the system exhibits its highest mechanical impedance is referred to as the system's natural frequency or resonance point. It is speculated that vibrationinduced disruptions in force perception may be particularly pronounced at the resonance frequency. Researchers have reported hand-arm system resonance in the range of 25 to $63 \mathrm{~Hz}$ (Kihlberg 1995, ISO 1998, Dong et al. 2004a). Dong et al. (2004b) reported that human fingers display resonance in the range of $100-250 \mathrm{~Hz}$. It is important to determine if vibration-induced disruptions in force perception and recall process may be particularly pronounced at the resonance frequencies. Resonance may also affect afferent sensory processes.

Studies have shown that increased muscle force increases the biodynamic system stiffness which, in turn, increases its mechanical impedance and its resonance frequency (Lundström 1984, Kihlberg 1995, Dong et al. 2004b). It follows then that increased grip and push forces might decrease the resonance effect and the vibration-induced sensorineural disturbances at certain frequencies. Theoretically, fewer disturbances should lead to increased force recall accuracy. Therefore, it is reasonable to anticipate an interaction between force level and the biodynamic response. 


\section{$2.4 \quad$ Force level effects}

Early magnitude production studies indicated that subjects generally tend to overestimate grip forces (Stevens and Mack 1959, Stevens 1960). Stevens and Mack (1959) asked their subjects to produce grip forces relative to randomly-ordered numbers. That is, the investigator would call out a number, then the subject would produce a grip force on a handgrip dynamometer that they thought corresponded to the number. In this fashion, they found that the responses approximated a power function with an exponent of 1.7. Based on these results, the researchers concluded that the perception of apparent grip force grows as the 1.7 power of the actual physical force applied to the dynamometer.

Stevens (1986) described a phenomenon that commonly occurs in matching experiments. This phenomenon exhibits itself as a tendency for the subject to shorten the range of their responses. In other words, the subject tends to err in the direction of the center of the scale. Stevens referred to this consequence as the "regression effect" where subjects tend to regress towards the mean. This effect revealed itself in several force-matching studies, as the results indicated that subjects typically produce pinch and grip force matches above the target at low-force levels while producing force matches below the target at the upper end of the test range (Kumar and Simmonds 1994, Kumar et al. 1994, Wiktorin et al. 1996).

In a second experiment in the Wiktorin et al. (1996) study, subjects were asked to generate predetermined pushing and pulling forces at five different force levels $(10 \mathrm{~N}, 50 \mathrm{~N}, 100 \mathrm{~N}$, 
$150 \mathrm{~N}$, and $300 \mathrm{~N})$. The forces were expressed in units familiar to the subjects $(\mathrm{kg})$. The subjects produced higher than requested forces at the three lowest targets, correct forces at $150 \mathrm{~N}$, and lower than requested forces at the $300 \mathrm{~N}$ target.

Similarly, subjects in the Wiker et al. (1989) study over-forced low-force grip targets, underforced high-force grip targets, and were accurate at mid-range grip targets. The researchers attributed this phenomenon to; a) diminished cutaneous feedback in hypoxic dermal tissues, resulting in greater reliance in muscle-tendon feedback for perceiving force, and b) loss of muscle contractility in high-force grips due to fatigue from previous gripping activity. Greater reliance on deep muscle-tendon signals to estimate grip or push forces leads to higher levels of force required to generate just noticeable differences in force perception. Up to a point, accuracy increased when subjects had to produce greater levels of perceived grip force.

Increased force levels may also facilitate fatigue effects. As workers grow tired, their perceptions of sensory and motor feedback may become altered. Indeed, fatigue has been shown to increase force-matching errors (Gooch and Randle 1993, Vincent et al. 2000). Gooch and Randle (1993) found that after a one-minute maximum voluntary contraction, subjects exerted significantly higher isometric forces when attempting to match a target force. In the Vincent et al. (2000) study, subjects attempted to match $30 \% \mathrm{MVC}$ targets on a stationary preacher curl apparatus following strenuous eccentric exercise. In one experimental condition, the subjects produced a target force with the exercised arm, and then they tried to match the force with their non-exercised (control) arm. In the second condition, the subjects used their control arms to produce the target force, and then attempted to match 
the force with their exercised arms. In both conditions, strenuous eccentric exercise was found to significantly affect the subjects' force-matching abilities.

Fatigue also seems to affect a subject's position sense as well as their perception of effort. A study by Heide and Molbech (1973) indicated that a sustained muscle contraction prior to attempts at position-matching might influence a person's ability to recall motor and sensory information and, in turn, hinder one's ability to duplicate a reference posture. Burgess and Jones (1997) discovered that perceived effort increases at a higher rate than perceived force as subjects become fatigued. The relationship between fatigue and psychophysical functions requires further study.

\subsection{Summary}

There is a need for hand force measuring techniques to be integrated into comprehensive risk assessments for HAVS, CTS, and other musculoskeletal disorders of the upper extremities. Psychophysical force-recall techniques may permit assessment of hand-tool coupling forces without the need for expensive or fragile instrumentation. Without a thorough understanding of the factors that can influence force-recall accuracy, these psychophysical techniques cannot be optimized. However, once these techniques are refined, force-recall methods can be used to characterize hand forces and may be incorporated into risk assessment strategies. Therefore, it is important to understand the effects of vibration and force level on force-recall accuracy and reliability before such methods are used in the field or in laboratory environments. 
Psychophysical techniques have been successfully used to evaluate workplace tasks involving the production of manual forces. The use of psychophysical tools such as Borg's scales has proven to be reliable for assessing exertion and discomfort associated with various postures and manual tasks. Studies have also shown that humans have an awareness of the sensations associated with limb movement and the application of various static and dynamic forces to stationary and mobile objects. Subjects are also able to reproduce postures, movements, and forces by relying on their memory of those sensations. Ergonomists have taken advantage of these reliable outcomes for analyzing lifting tasks, evaluating risks of musculoskeletal injuries and disorders, and for the design of safer workstations, task postures, and work procedures.

Perception and recall of manual forces and position sense appear to be functions with both efferent and afferent components. Efferent signals control which muscles become involved with a specific action, as well as the magnitudes of muscle tension or motor forces. While efferent signals seem to dominate, afferent components also play a significant role in force production and force perception. The afferent information can be divided into muscle afferents and cutaneous afferents. The muscle afferent information is produced by peripheral receptors in the muscles, joints, and tendons that provide feedback to the sensory cortex. These transmissions contain information regarding muscle extension, contraction, and tension along with joint position. The cutaneous afferent signals from the hand carry information regarding textures, sizes, shapes, and pressures imposed on the skin and subcutaneous tissues.

Studies indicate that vibration may effect both the efferent and the afferent components of force perception and recall. There is evidence that vibration can affect efferent transmissions 
and both muscle and cutaneous afferent signals. There is also evidence to indicate that vibration can affect a subject's memory of perceived forces.

Another potential factor is the vibration-induced phenomenon known as the tonic vibration reflex. Researchers have discovered that involuntary muscle contractions can be initiated by certain vibration exposures under certain conditions. Based on previous research, it seems unlikely that a voluntary power grip or palm push effort could be affected by a reflex action initiated through hand-transmitted vibration. Moreover, early TVR studies showed that subjects could counteract or prevent TVR actions in the presence of visual feedback of limb position or muscle force. While it seems unlikely that TVR activity will influence the outcomes of this research, this phenomenon cannot be ignored as a potential factor affecting force-recall accuracy.

The human hand-arm system and its sub-systems respond differently to various vibration exposures and with different levels of muscle activity. The system's response to vibration can affect the motions and stresses of the various tissues involved in force production and perception. Thus, the biodynamic response of the hand-arm systems and sub-systems may affect the perception and recall of manual forces. Vibration-induced disruptions in force perception and recall may be particularly pronounced at the resonance frequencies of the various systems and sub-systems. Resonance may also affect efferent and afferent sensory processes. Hand-arm system resonance has been identified to be in the range of 25 to $63 \mathrm{~Hz}$. The fingers display resonance in the range of 100 to $250 \mathrm{~Hz}$. Thus, these frequency ranges are of particular interest as potential influences on force-recall accuracy. 
Several studies have indicated that force level is a significant factor as regards force perception and recall. There have been several reports that subjects tend to over-force low-force targets and under-force high-force targets. However, there are several findings that indicate that subjects can reliably recall and reproduce forces over a wide range of postures and force levels. Studies have shown that increased muscle force increases the biodynamic system stiffness which, in turn, increases its mechanical impedance and its resonance frequency. Thus, increased or decreased grip and push forces might influence the resonance effect and the vibration-induced sensorineural disturbances at certain frequencies. Therefore, there is likely an interaction between force level and hand and arm system resonance. Increased force levels may also facilitate fatigue effects, and in turn, affect a subject's force perception and recall ability. 


\section{H A P T E R 3: M E T H O D S}

\subsection{General study description}

As stated in the introduction, the major objective of this research is to provide information regarding specific factors that may influence the accuracy of force-recall procedures that may be used to estimate grip and push forces applied to vibrating tools. The three primary specific aims of this research are:

(i) to examine the effects of vibration frequency on a subject's ability to recall and duplicate target grip and push forces applied to an instrumented handle;

(ii) to examine the effects of vibration magnitude on a subject's ability to recall and duplicate target grip and push forces applied to an instrumented handle; and

(iii) to examine the effects of grip and push force magnitude on a subject's ability to recall and duplicate target grip and push forces applied to an instrumented handle.

Two experiments using human subjects were completed to meet these specific aims. In each experiment, the subject was instructed to apply specified grip and push forces to a vibrating, instrumented handle for 45 seconds. Visual feedback of the applied forces was provided during this vibration exposure period. After a short, controlled rest period, the subject was asked to recall and re-apply the grip and push forces to an idle handle without the benefit of visual feedback. The vibration conditions (frequency and magnitude) were varied as were 
the target grip and push force levels. The accuracies of their recall/duplication attempts were analyzed to examine the effects of single-axis, sinusoidal vibration and grip and push force level.

\subsection{Protection of the human subjects}

\subsubsection{Protocol IRB approval / study location}

In order to protect the rights and welfare of the human subjects, the study protocol was submitted for review and approval to the National Institute for Occupational Safety and Health (NIOSH) Human Subjects Review Board (HSRB) as well as the West Virginia University Institutional Review Board (IRB). The approval notices are attached as Appendix

A. Once the protocol achieved IRB approval, the proposed experiments were conducted in the Morgantown NIOSH facility, 1095 Willowdale Road, Morgantown, West Virginia, in the Human Vibration Laboratory (room L-B206).

\subsubsection{Subject recruitment and reimbursement}

Most of the study participants were recruited from West Virginia University through recruiting announcements (Appendix B), which were posted on bulletin boards around the campus. The recruiting announcement instructed the potential participants to contact the principal investigator if they were interested. At that time, the principal investigator explained the study in more detail and answered any questions. Each participant (prior to his/her recruitment) was asked, whether he/she had ever encountered any form of hand-arm vibration syndrome, such as tingling, numbness or visible white patches over the skin of the hand. Subjects with a history of HAVS, as determined from such symptoms, were to be 
excluded from the study. However, none of the volunteers reported such symptoms, and no volunteers were excluded from the study.

As compensation for their time, each participant was reimbursed $\$ 45.00$ for each testing session. All subjects were asked to complete two testing sessions. Each subject was advised of the right to discontinue the testing at his/her own will. The subjects were advised that in cases where the subject opted to discontinue testing before a session was completed, the participant would be paid on a pro-rated basis at the rate of $\$ 15.00 /$ hour.

\subsubsection{Risks to study participants}

The risks for the test subjects were minimal. The testing was non-invasive and only entailed grasping a vibrating handle for short periods of time. The total vibration exposure for each test subject did not exceed 30 minutes per test day. The vibration exposures were comfortably below the daily exposure limit published in the ANSI S3.34 standard (ANSI 1986). Vibration exposures at the levels used in this study have been shown to be safe. Several laboratories throughout the world perform similar tests routinely. Prior to these experiments, twelve subjects participated in a series of tests within a similar NIOSH HSRBapproved study (HSRB 02-HELD-09XP). No subjects reported any discomfort or problems associated with the previous testing.

While no adverse health effects to the test subjects were anticipated, the subjects were warned of the possibility that some test subjects may experience tingling in the hand and 
fingers immediately after exposure, but that this tingling would not last more than a few seconds. They were further warned that there was also a slight chance of some minor muscle soreness in the hand and arm, but that this soreness would not last more than 24 hours and would resolve itself without any medical treatment.

\subsubsection{Informed consent procedures}

Each subject was asked to read and sign a consent form (Appendix C) before participating in the study. Each subject was given adequate time to read the document and to ask questions before signing it. Each participant received a copy of the consent document (signed by the investigator and the participant) upon request. Subjects were informed that their participation in the study was strictly voluntary, and that they could leave the study at any time and for any reason. Each subject was given the name and phone number of the chair of the NIOSH Human Subjects Review Board in case of any questions or concerns about the study.

\subsubsection{Confidentiality}

The identity of the test subjects and any specific information derived from their participation in the study was and will continue to be kept confidential to the extent allowed by law. At the start of the test, each participant was assigned a random alphanumeric identifier. This identifier was used to track the test results. The names of the test subjects or any other facts that might point to their identity do not appear anywhere on the test data, in this dissertation, or in any published results. The records are kept under lock, and only the investigators involved in the study have access to them. 


\subsubsection{Emergency procedures}

Emergency procedures were established for the handling of the event of an emergency. Briefly, the investigators were instructed to call NIOSH Security and inform them of the location and the nature of the problem. In the event of a serious problem, 911 (in addition to NIOSH Security) was to be called directly. The investigators were instructed to remain with the test subject until help arrived.

\subsection{Common setup and strategy for the two experiments}

Anthropometric measurements were taken for each subject in both experiments. Data collected included the subject's gender, age, weight, stature, hand breadth at the metacarpals, hand length, hand circumference at the metacarpals, hand volume, and forearm volume. The volume measurements were based on the amount of water displaced by the submerged body segments.

The general approach to evaluating the force-recall technique was to conduct two experiments designed to assess the impact of single-axis, sinusoidal vibration and different levels of applied hand force on a human's ability to reproduce quasi-steady state grip and push forces. In each experiment, a vibration test system (Unholtz-Dickie, TA250-S032-PB) was programmed to deliver single-axis, sinusoidal vibration to an instrumented handle fixed on a shaker. Vibration was presented to the handle along the axis of the forearm at specific amplitudes for given exposure frequencies. The vibration frequencies selected for the two experiments cover a range of exposures that could be anticipated in typical work tasks involving vibrating tools. For an evaluation of recalled forces to be meaningful, exposure conditions of the force 
memorization period need to be controlled to the best possible extent. In these experiments, the single-axis vibration frequency and magnitude exposures were controlled through a servo control system. Handle vibration was further scrutinized via the vibration data acquisition system (B\&K 2816). Hence, vibration exposures were meticulously regulated and monitored throughout both experiments.

Each study participant stood upright on the calibrated force plate mounted on a platform. The height of the platform was adjusted so that the subject could apply a power grip to the instrumented cylindrical handle at its midpoint while keeping their forearm parallel to the floor. With the elbow angled at $90^{\circ}$, the subject applied specified hand forces (grip and push) to the vibrating handle. Three levels of hand force were studied in each experiment; a) 15-N grip/25-N push, b) 30-N grip/50-N push, and c) $45-\mathrm{N}$ grip/75-N push. The subject posture and hand force levels were based on those presented in the ISO standard for the measurement of anti-vibration glove transmissibility (ISO 10819 1996). The general test setup and subject posture are depicted in Figure 3.3.1 and Figure 3.3.2. 


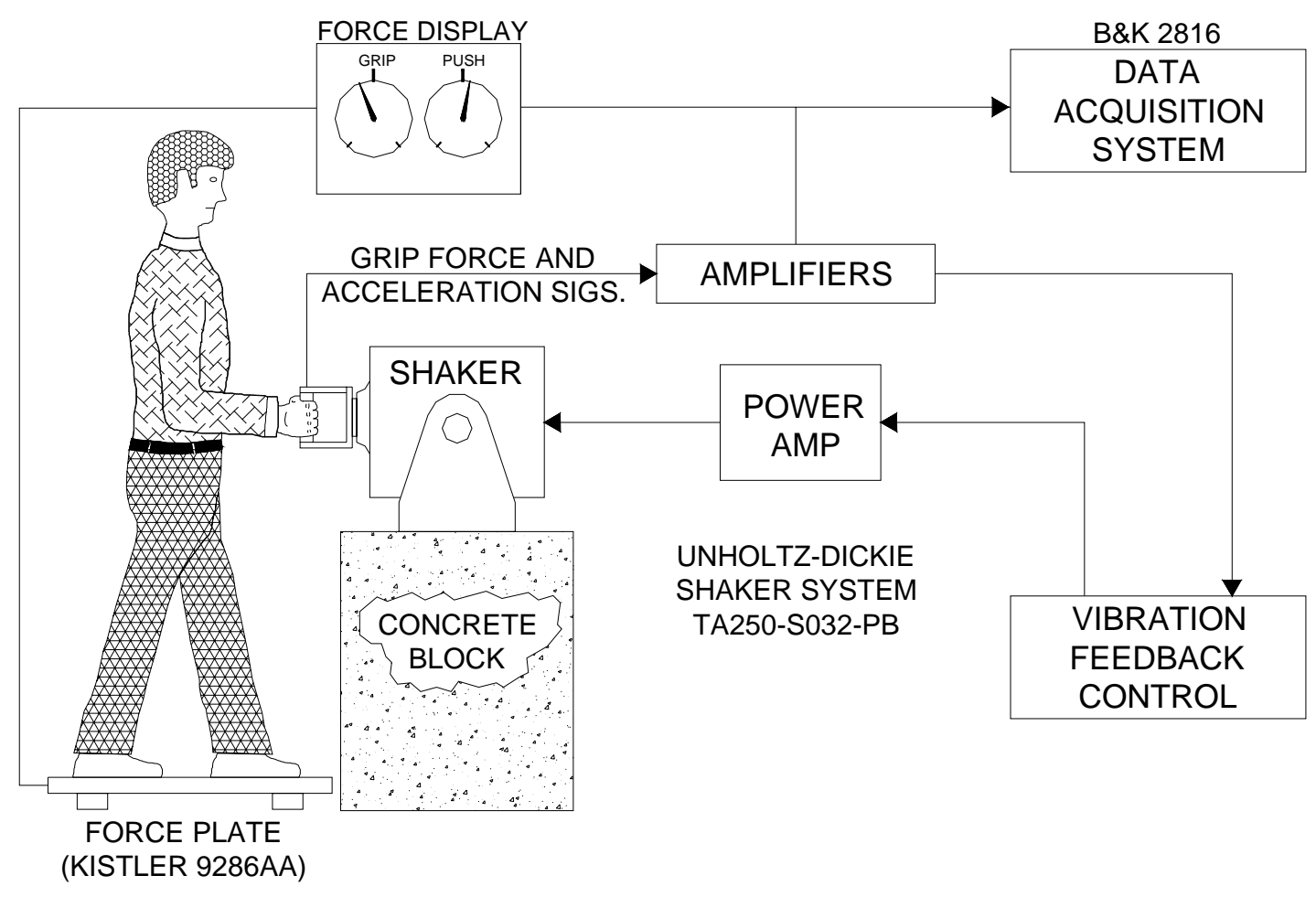

Figure 3.3.1 Subject posture and experimental setup for the study.

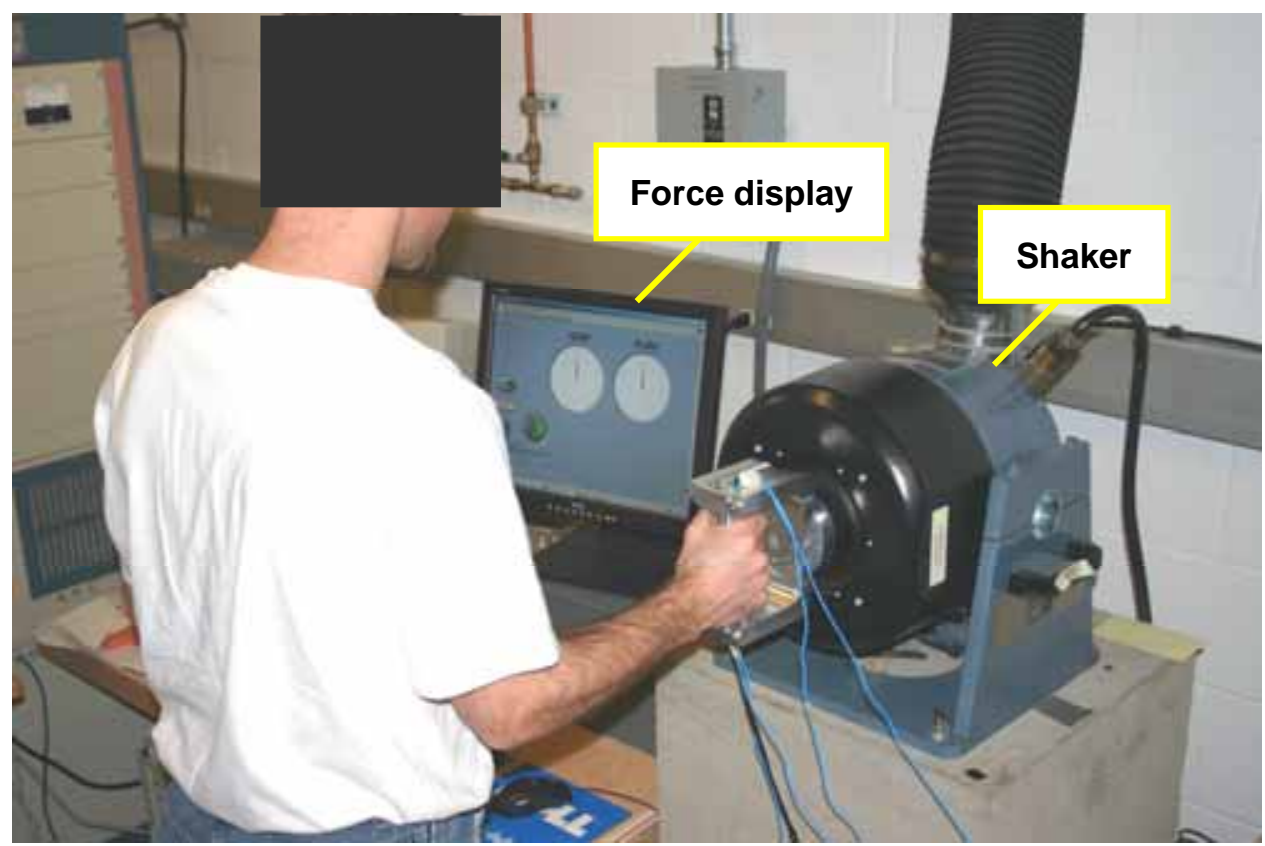

Figure 3.3.2 Subject posture during the vibration exposure/force memorization phase of a typical trial. 
A schematic of the instrumented handle is provided in Figure 3.3.3. Signals from two calibrated strain gauge force sensors (Interface, SML-50) were amplified, averaged, and passed into a data acquisition module (National Instruments, PCI-6036E) to record handle grip force. Handle push forces were quantified by recording horizontal foot ground-reaction forces on a force plate (Kistler, 9286AA).

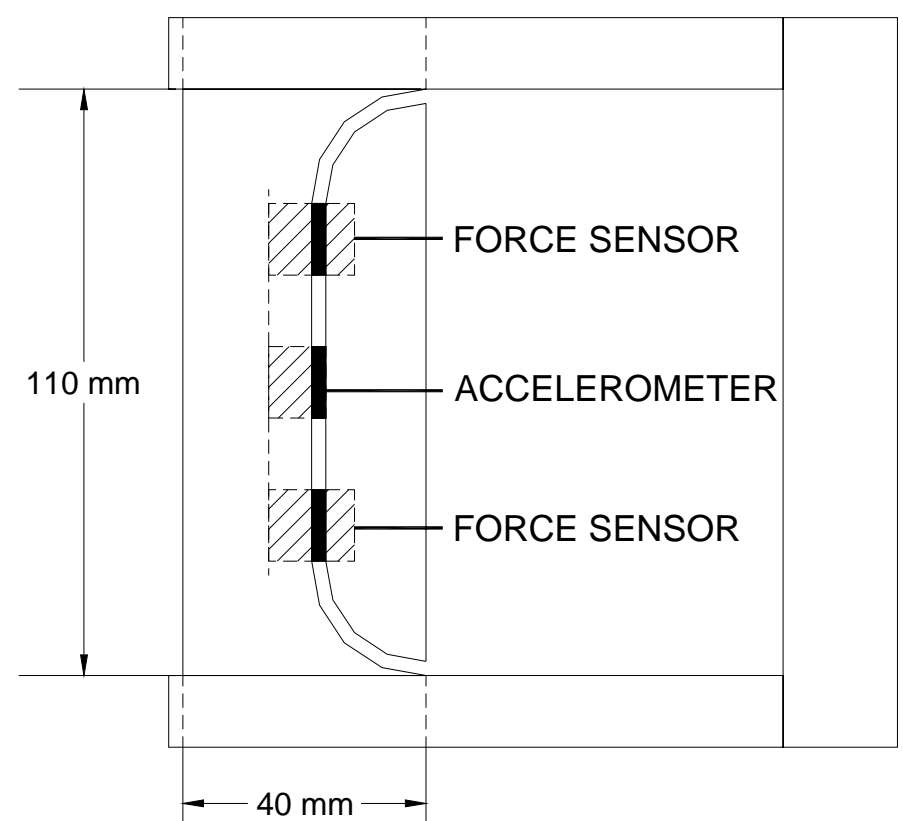

Figure 3.3.3 Schematic of the instrumented handle.

To provide visual feedback of the applied grip and push forces, a custom graphical display was developed using LabVIEW ${ }^{\mathrm{TM}}$ software (National Instruments, version 6.1). The grip and push force feedback screen is shown in Figure 3.3.4. As shown in the figure, grip and push forces were simultaneously displayed in front of the subjects on a computer monitor as 
unnumbered dial gauges. The top of each dial gauge had an index mark to indicate the target force. The grip force dial was programmed to display a range of target force $\pm 15 \mathrm{~N}$, and the push force dial was set at target force $\pm 25 \mathrm{~N}$. Regardless of the values of the target forces, the goal was to orient the needles vertically. The grip and push force signals were sampled at $1,000 \mathrm{~Hz}$, and the video display was refreshed at a rate of $4 \mathrm{~Hz}$.

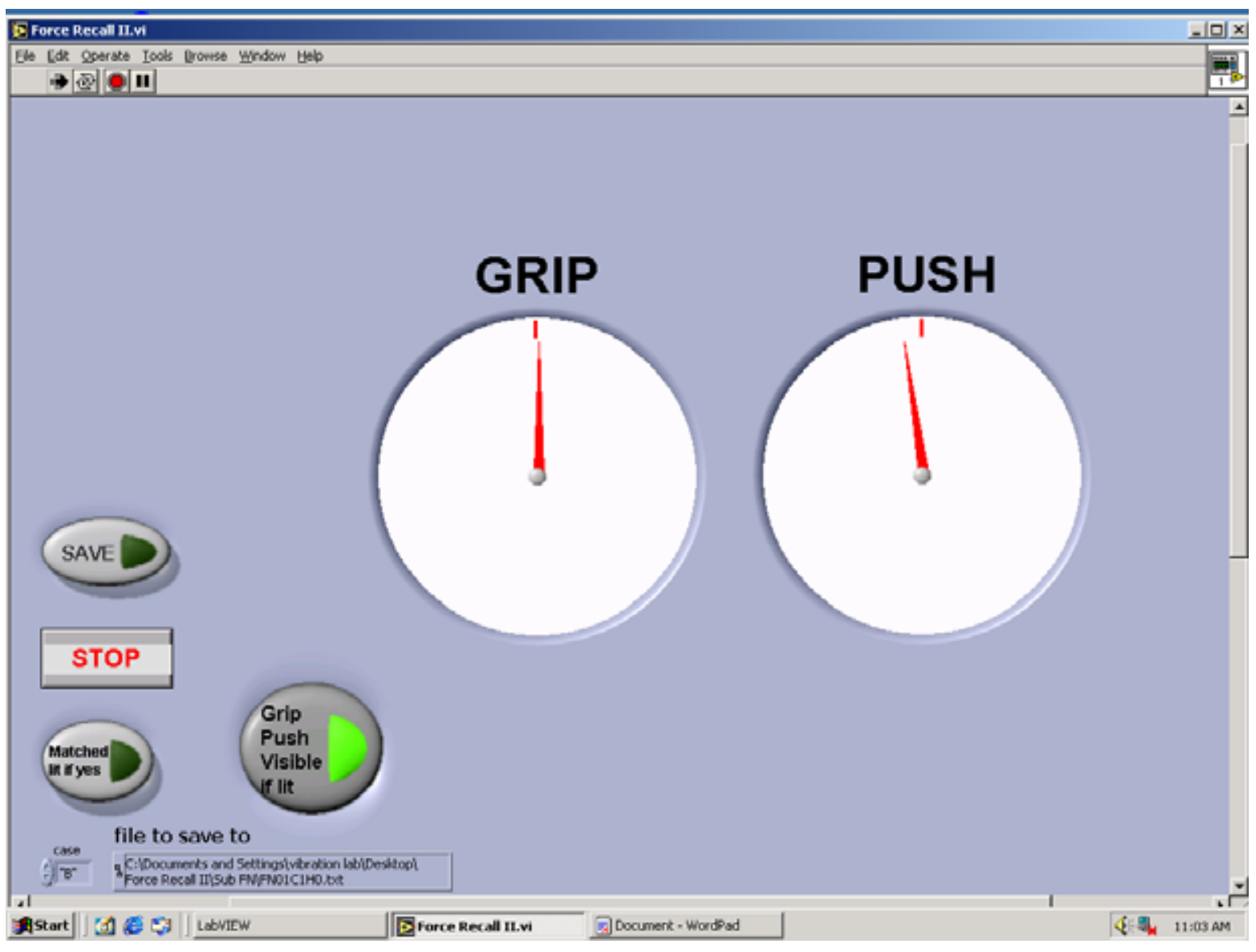

Figure 3.3.4 Grip and push force feedback display on a computer monitor. 
The subjects were instructed to apply specific grip and push forces to the instrumented handle. The instruction set is attached as Appendix D. The subjects were provided with visual feedback while they attempted to "memorize" the applied grip and push forces. At the conclusion of the memorization period and a controlled rest period, the subjects were asked to recall and duplicate the grip and push forces without the aid of visual feedback. The test conditions were varied in order to determine the significance of common workplace factors. The primary factors studied in the two experiments were the effects of grip and push force magnitude, vibration frequency, and vibration magnitude.

\subsection{Experiment 1}

\subsubsection{Subjects}

For Experiment 1, twelve volunteer male subjects were recruited from West Virginia University and participated in the study on a paid and informed-consent basis as described above.

\subsubsection{Vibration exposure conditions for Experiment 1}

In this experiment, subjects were exposed to five vibration conditions consisting of singleaxis sinusoidal vibrations along the axis of the forearm at amplitudes for given exposure frequencies that met the ANSI S3.34 Standard (ANSI 1986) less than 0.5-hour exposure limits. Table 3.4.1 provides the handle vibration frequency and acceleration characteristics for vibration exposures in Experiment 1. 
Table 3.4.1 Single-axis sinusoidal vibration exposures used in Experiment 1.

\begin{tabular}{cc}
\hline $\begin{array}{c}\text { Frequency } \\
(\mathrm{Hz})\end{array}$ & Acceleration $\left(\mathrm{m} / \mathrm{s}^{2}\right.$ peak $)$ \\
\hline 0 & - \\
12.5 & 11.3 \\
40 & 28.4 \\
125 & 89.9 \\
250 & 179.4 \\
\hline
\end{tabular}

\subsubsection{Experiment 1 Procedure}

Prior to participation, the study procedure was explained to each subject (see Appendix D). Following the briefing, each subject read and signed a consent form (see Appendix C).

During each trial, the subject was provided with visual feedback of their grip and push forces while they attempted to "memorize" their applied forces. At the conclusion of a 45-second force production/memorization period, subjects were instructed to release the handle which initiated a short, controlled rest period of either $10 \mathrm{~s}$ or $20 \mathrm{~s}$. During the rest period, the shaker was turned off, and the force dials were blanked from the monitor.

At the end of the rest period, the subject was instructed to re-grip the now-idle handle and attempt to reproduce the grip and push forces without the aid of visual feedback. The subject was asked to nod their head once they believed they had matched the grip and push forces produced during exposure and to maintain the matched forces for $10 \mathrm{~s}$. When signaled by the subject, the investigator mouse-clicked an icon on the feedback monitor to flag the data and initiate the force-recall measurement period. After the ten-second grip and push force-recall 
period, subjects were instructed to release the handle, step off the platform, and to rest for 90 s. During the last $10 \mathrm{~s}$ of the rest period, vibration was re-applied to the handle. Subjects were then asked to step back on the platform, re-assume the correct posture, and prepare for the next trial. The timeline of a complete force memorization, recall, and rest sequence is depicted in Figure 3.4.1. The top line of the figure depicts the status of the handle and feedback monitor, while the bottom line represents subject activity.
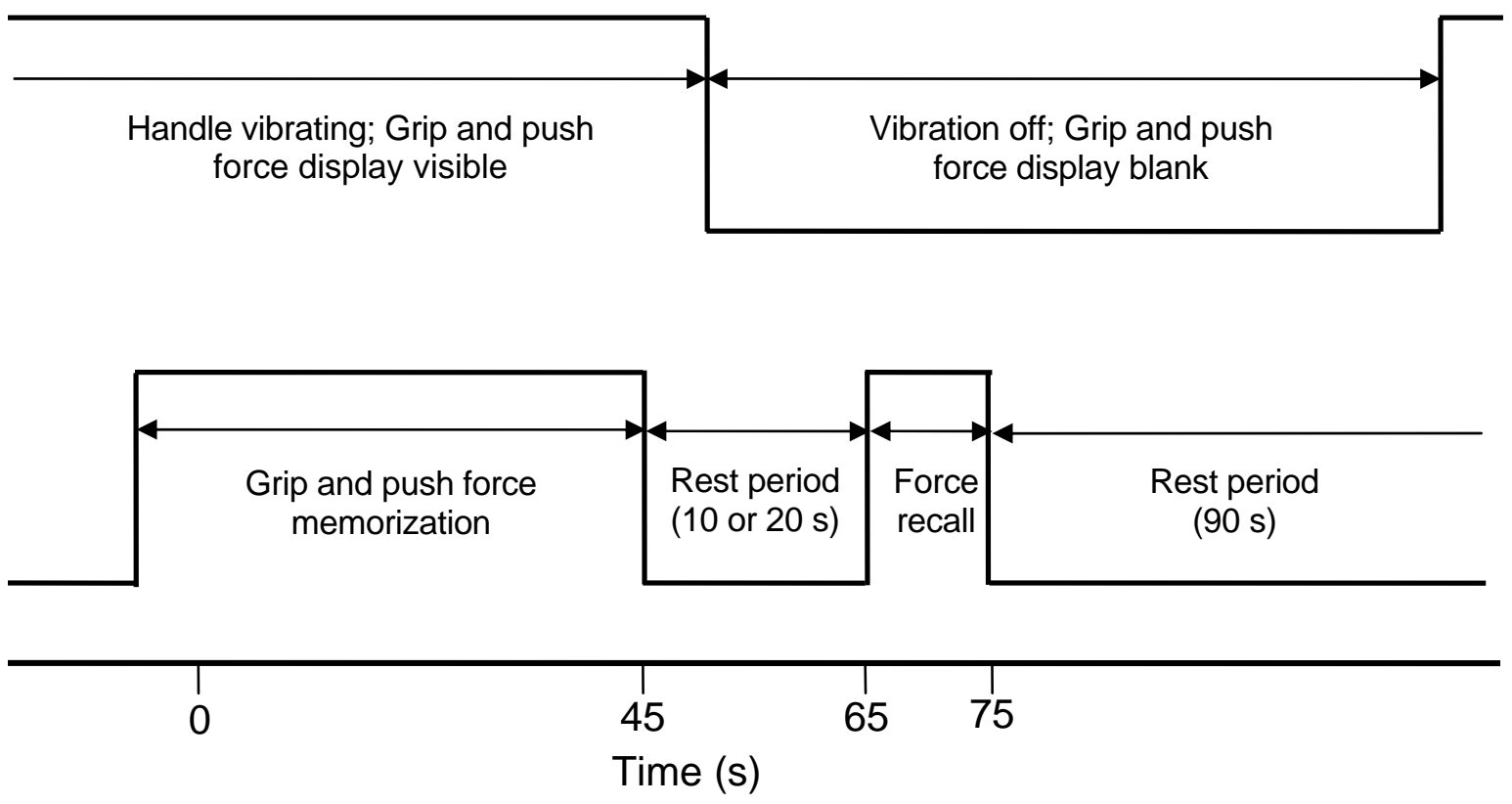

Figure 3.4.1 Timeline of events during a force memorization and recall trial in Experiment 1.

Each subject completed three practice trials at the beginning of each test session. Following practice, subjects completed a 30-trial matrix that consisted of each combination of frequency (five levels), force (three levels), and rest period (two levels). The 30-trial sequence was completely randomized for each subject. At no time were the subjects given 
information regarding the vibration frequency, hand-force levels, or duration of the rest period. After the twelfth and twenty-fourth trials, subjects were given five-minute rest breaks. Including time for procedure explanation and consent form signing, a test session lasted about $2 \frac{1}{2}$ hours. In order to examine repeatability, each of the 12 subjects returned for a second session of testing. There was a minimum of one day and a maximum of seven days between the two sessions. The same vibration frequency, force level, and rest period combinations were completed, but with a different independently randomized trial sequence. Hence, by the end of the study, each of the 12 male subjects had twice completed the 30-trial matrix.

\subsubsection{Experiment 1 Data Analysis}

Grip and push forces were averaged over the 45 -second force production/memorization period. These averages became the values to which the averaged force-recall values were compared. It was observed that the recall forces produced by some of the subjects began to decay about midway through each force-recall attempt. Therefore, only the first $3 \mathrm{~s}$ of the force-recall attempt were used during data analysis. Force-recall accuracy was quantified in terms of error. The force-recall error was computed for each trial from

$$
E=F_{r}-F_{m}
$$

where $E$ is the error between the average force produced during the 45 -s vibration exposure/memorization period $\left(F_{m}\right)$ and the 3 -s force-recall average $\left(F_{r}\right)$. Error values were expressed in Newtons. 
An Analysis of Variance (ANOVA) was conducted to identify influential factors and any significant interactions. The dependent variable was error $(E)$ as defined above. A full factorial, general linear model (GLM) was used with exertion type (grip or push), vibration frequency (5 levels), applied force (3 levels), rest period between force memorization and recall trials (10 s or $20 \mathrm{~s}$ ), and test session (Day 1 or Day 2) as fixed effects. Tukey’s Honestly Significant Difference (HSD) tests were used for post-hoc analysis of significant effects $(p \leq 0.05)$ using 95\% confidence intervals. Test-retest reliabilities of replicated trials were computed using Pearson product-moment correlations.

Statistical power for main effects was analyzed in terms of experimental sensitivity using the following formula adapted from Montgomery (2001):

$$
D=\sqrt{\frac{2 a \sigma^{2} \phi^{2}}{n}}
$$

where $D$ is the experiment's sensitivity for detecting response variable differences (force recall error expressed in Newtons); $a$ is the number of levels for the main factor of interest; $\sigma^{2}$ is the variance of the response variable; $\phi^{2}$ is an estimate of non-centrality obtained from tables of operating characteristic curves of type II error probability (Pearson and Hartley 1972) as presented in Montgomery (2001); and $n$ is the number of replicates at each level of the factor of interest. Type II error was evaluated at three power levels $(1-\beta=0.70,0.80$, and 0.90$)$ with alpha fixed at 0.05 . 
Similarly, for evaluating statistical power for two-way interactions, the following formula (also adapted from Montgomery 2001) was used:

$$
D=\sqrt{\frac{2 \sigma^{2} \phi^{2}[(a-1)(b-1)+1]}{n}}
$$

where $a$ and $b$ are the number of levels for the two interaction terms of interest Once again, type II error was evaluated at three power levels $(1-\beta=0.70,0.80$, and 0.90$)$ with alpha fixed at 0.05 .

\subsection{Experiment 2}

\subsubsection{Subjects}

For Experiment 2, 20 volunteer subjects (10 male, 10 female) were recruited via posted announcements at West Virginia University and other local public facilities. The subjects participated in the study on a paid and informed-consent basis as described above.

\subsubsection{Vibration Exposure Conditions for Experiment 2}

Unlike Experiment 1, Experiment 2 examined two levels of vibration magnitude. The levels chosen represent the extremes of the exposure range to which a user of typical vibratory tools would be exposed. The two levels of vibration magnitude correspond to the less than 0.5 -hour and 4-8-hour limits established by the ANSI S3.34 Standard (ANSI 1986). The vibration frequencies used in Experiment 2 were octaves ranging from $16 \mathrm{~Hz}$ to $125 \mathrm{~Hz}$. Including the static condition $(0 \mathrm{~Hz})$, there were nine different vibration exposure conditions for Experiment 
2. The vibration frequencies and magnitudes are listed in Table 3.5.1. The ANSI limits for each of the four octaves are depicted in Figure 3.5.1.

Table 3.5.1 Single-axis sinusoidal vibration used during Experiment 2.

\begin{tabular}{ccc}
\hline $\begin{array}{c}\text { Frequency } \\
(\mathrm{Hz})\end{array}$ & $\begin{array}{c}\text { Magnitude Level 1 } \\
\left(\mathrm{m} / \mathrm{s}^{2} \text { peak }\right)\end{array}$ & $\begin{array}{c}\text { Magnitude Level 2 } \\
\left(\mathrm{m} / \mathrm{s}^{2} \text { peak }\right)\end{array}$ \\
\hline 0.0 & - & - \\
16.0 & 2.8 & 11.3 \\
31.5 & 5.6 & 22.6 \\
63.0 & 11.3 & 45.1 \\
125.0 & 22.5 & 89.9 \\
\hline
\end{tabular}

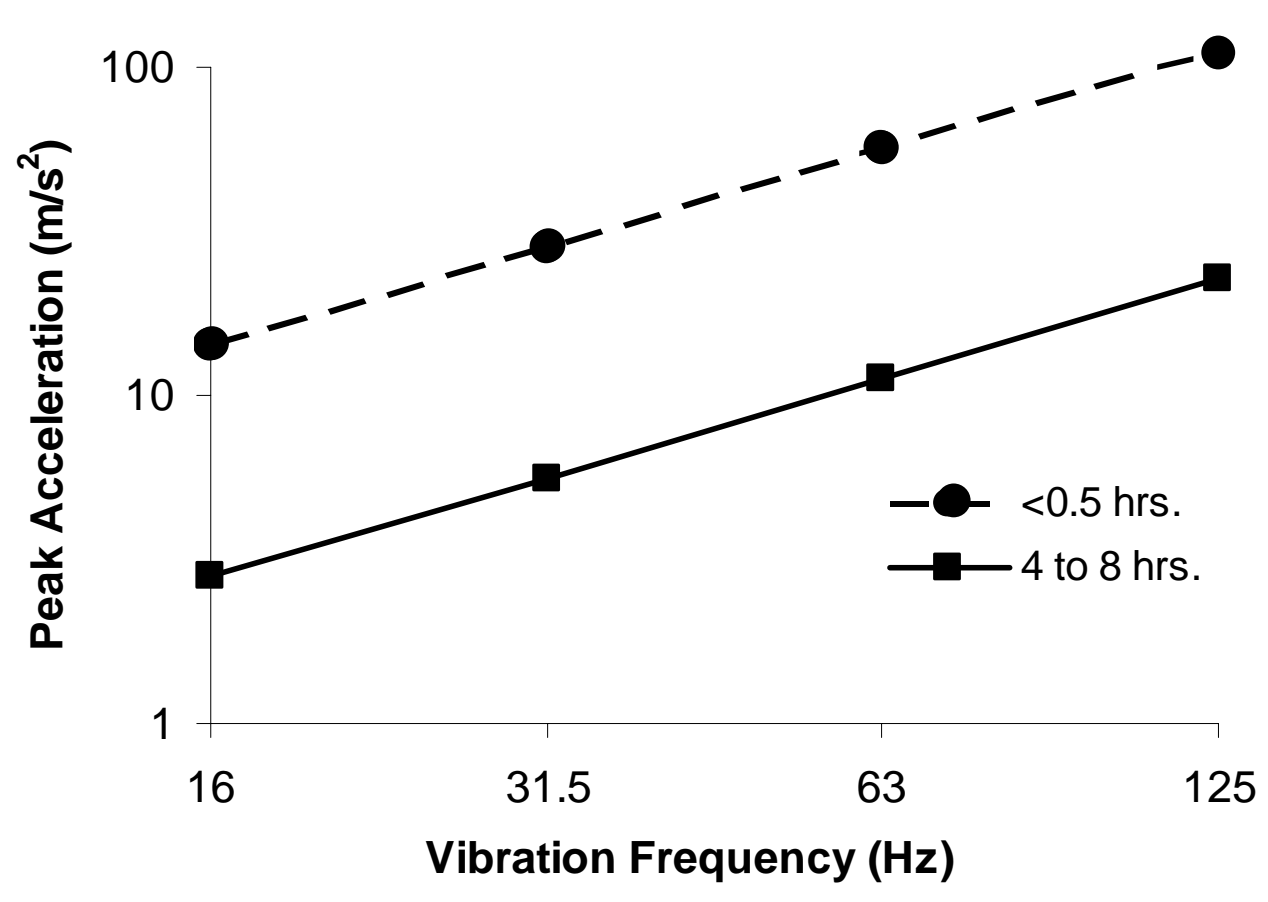

Figure 3.5.1 ANSI limits of daily vibration exposure for the four vibration frequencies used in Experiment 2 (ANSI S3.34, 1986). 


\subsubsection{Experiment 2 Procedure}

The procedure for Experiment 2 varied only slightly from Experiment 1 . The differences were:

a) changed vibration frequency exposures,

b) two levels of vibration magnitude (instead of one),

c) one level of rest period duration (instead of two), and

d) the force recall measurement period was reduced from $10 \mathrm{~s}$ to $5 \mathrm{~s}$.

The same three levels of grip and push forces that were used in Experiment 1 were used in Experiment 2 .

Prior to participation, the study procedure was explained to each subject just as in Experiment 1 (refer to Appendix D for the instruction set). Likewise, following the briefing, each subject read and signed a consent form (see Appendix C). With the exceptions noted above, the procedure for Experiment 2 was identical to Experiment 1. Briefly, each subject stood upright on the calibrated force plate mounted on a platform. The height of the platform was adjusted so that the subject could apply a power grip to the instrumented cylindrical handle at its midpoint while keeping their forearm parallel to the floor. With the elbow angled at 90 degrees, the subject applied specified grip and push forces to the vibrating handle. Each subject was provided with visual feedback of their grip and push forces while they attempted to "memorize" the applied forces. At the conclusion of a 45-second force production and memorization period, the subject released the handle which initiated a controlled rest period of $20 \mathrm{~s}$. During the rest period, the shaker was turned off, and the force dials were blanked from the monitor. At the end of the $20 \mathrm{~s}$ rest period, the subject re-gripped the now-idle handle and attempted to reproduce the grip and push forces without the aid of visual feedback. Just as in 
Experiment 1, the subject was asked to nod their head once they believed that the grip and push forces matched those produced during vibration exposure. The subject was instructed to maintain the matched grip and push forces for $5 \mathrm{~s}$. After the five-second grip and push forcerecall period, the subject released the handle, stepped off the platform and rested for $90 \mathrm{~s}$. During the last $10 \mathrm{~s}$ of the 90 -s rest period, vibration was re-applied to the handle, and the force dials were reactivated in preparation for the subsequent trial. The subject then stepped back on the platform, re-assumed the correct posture, and prepared for the next trial. The timeline of a complete force memorization, recall, and rest sequence for Experiment 2 is depicted in Figure 3.5.2. The top line of the figure depicts the status of the handle and feedback monitor, while the bottom line represents subject activity.
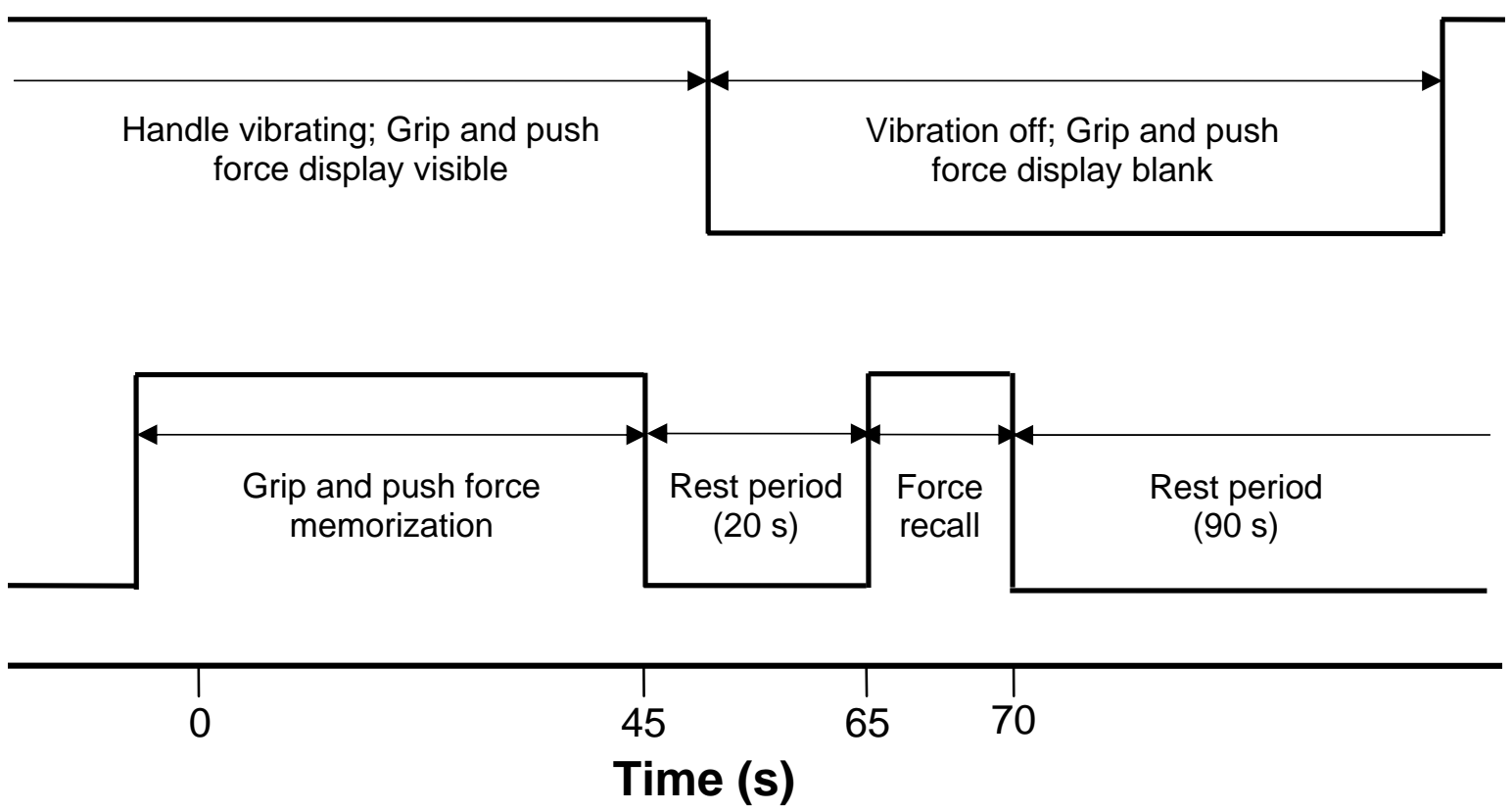

Figure 3.5.2 Timeline of events during a force memorization and recall trial in Experiment 2. 
As in Experiment 1, each subject completed three practice trials at the beginning of each test session. Following the three practice trials, subjects completed a 27-trial matrix that consisted of each combination of vibration exposure condition (nine levels) and force level (three levels). The trial sequence was completely randomized for each subject. At no time were the subjects given information regarding the vibration frequency, vibration magnitude, or hand-force levels. After the tenth and twentieth trials, each subject was given five-minute rest breaks. Including time for procedure explanation and consent form signing, each 30-trial test session lasted about $2 \frac{1}{2}$ hours. In order to examine repeatability, each of the subjects returned for a second session of testing. During the second session, the same vibration frequency, vibration magnitude, and force level combinations were completed, but with a different independently randomized trial sequence.

Subject reimbursement was the same for both experiments. As stated above, each participant was reimbursed $\$ 45.00$ for each session. Each subject was advised of the right to discontinue the testing at their own will.

\subsubsection{Experiment 2 Data Analysis}

Just as in Experiment 1, grip and push forces were averaged over the 45 -second force production/memorization period. These averages became the values to which the averaged

force recall values were compared. As mentioned above, it was observed during Experiment 1 that the recall forces produced by some of the subjects began to decay about midway through some of the 10-second force-recall attempts. Therefore, the force recall period was 
shortened to $5 \mathrm{~s}$ in Experiment 2. For the analysis for both experiments, only the first $3 \mathrm{~s}$ of the force-recall period were used. As in Experiment 1, force-recall accuracy was quantified in terms of error. The force-recall error was computed for each trial from Equation 3-1.

An ANOVA was conducted to identify influential factors and any significant interactions for Experiment 2. Once again, a full factorial GLM was used with exertion type (grip or push), vibration frequency (5 levels), vibration magnitude (2 levels), applied force (3 levels), gender, and test session (Day 1 or Day 2) as fixed effects. Tukey's HSD tests were used for post-hoc analysis of significant effects $(\mathrm{p} \leq 0.05)$ using $95 \%$ confidence intervals. Test-retest reliabilities of replicated trials were computed using Pearson product-moment correlations.

Statistical power for Experiment 2 was analyzed in the same fashion as Experiment 1. Equation 3-2 was used to evaluate main effects while Equation 3-3 was used to evaluate twoway interactions. 
C H A P T E R 4: R E S U L T S

\subsection{Experiment 1 results}

\subsubsection{Subject descriptors for Experiment 1}

Table 4.1.1 contains descriptors for the 12 male subjects who participated in Experiment 1 .

Table 4.1.1 Descriptors of the twelve male participants of Experiment 1.

\begin{tabular}{|c|c|c|c|c|c|c|c|c|}
\hline Subject & $\begin{array}{l}\text { Age } \\
\text { (yrs) }\end{array}$ & $\begin{array}{c}\text { Stature } \\
(\mathrm{m})\end{array}$ & $\begin{array}{c}\text { Weight } \\
(\mathrm{kg})\end{array}$ & $\begin{array}{c}{ }^{1} \text { Hand } \\
\text { breadth } \\
(\mathrm{mm})\end{array}$ & $\begin{array}{l}{ }^{2} \text { Hand } \\
\text { length } \\
(\mathrm{mm})\end{array}$ & $\begin{array}{c}{ }^{3} \text { Hand } \\
\text { circum. } \\
(\mathrm{mm})\end{array}$ & $\begin{array}{c}{ }^{4} \text { Hand } \\
\text { volume } \\
(\mathrm{ml})\end{array}$ & $\begin{array}{c}{ }^{5} \text { Forearm } \\
\text { volume } \\
(\mathrm{ml})\end{array}$ \\
\hline 1 & 27 & 1.80 & 86.2 & 91 & 188 & 220 & 400 & 1820 \\
\hline 2 & 24 & 1.80 & 81.7 & 84 & 180 & 220 & 360 & 1500 \\
\hline 3 & 22 & 1.91 & 90.7 & 85 & 191 & 211 & 420 & 1700 \\
\hline 4 & 27 & 1.73 & 97.7 & 95 & 206 & 233 & 520 & 1970 \\
\hline 5 & 24 & 1.77 & 115.8 & 88 & 197 & 219 & 460 & 2320 \\
\hline 6 & 18 & 1.73 & 63.5 & 87 & 184 & 213 & 350 & 1340 \\
\hline 7 & 25 & 1.91 & 104.3 & 98 & 200 & 242 & 500 & 2080 \\
\hline 8 & 19 & 1.78 & 90.7 & 89 & 196 & 215 & 420 & 1780 \\
\hline 9 & 23 & 1.85 & 86.2 & 89 & 194 & 222 & 450 & 1760 \\
\hline 10 & 23 & 1.82 & 83.9 & 82 & 193 & 210 & 430 & 1660 \\
\hline 11 & 28 & 1.80 & 72.8 & 87 & 195 & 210 & 395 & 1460 \\
\hline 12 & 20 & 1.83 & 74.8 & 77 & 187 & 203 & 400 & 1680 \\
\hline Mean & 23 & 1.81 & 87.4 & 88 & 193 & 218 & 425 & 1756 \\
\hline Min & 18 & 1.73 & 63.5 & 77 & 180 & 203 & 350 & 1340 \\
\hline Max & 28 & 1.91 & 115.8 & 98 & 206 & 242 & 520 & 2320 \\
\hline SD & 3.2 & 0.06 & 14.2 & 5.6 & 7.2 & 10.7 & 51.0 & 272.7 \\
\hline $\begin{array}{l}{ }^{1} \text { at metac } \\
2 \text { from tip } \\
3 \text { at metac } \\
4 \text { water di } \\
{ }_{5} \text {. }\end{array}$ & $\begin{array}{l}\text { arpals } \\
\text { of 3rd } \\
\text { arpals }\end{array}$ & finger & rease & rist & & 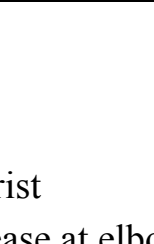 & & \\
\hline
\end{tabular}




\subsubsection{Recalled grip and push force distributions for Experiment 1}

Table 4.1.2 and Figure 4.1.1 provide information regarding the subjects' abilities to recall and reproduce the memorized grip and push forces during Experiment 1. The box and whisker plots shown in Figure 4.1.1 display the distribution of the subjects' grip and push force-recall attempts at each force level. The box represents the interquartile range of the responses while the upper and lower whiskers extend to the $90^{\text {th }}$ and $10^{\text {th }}$ percentiles, respectively. The median response value for each group is also indicated in the figure.

Table 4.1.2 Recalled grip and push forces (in Newtons) across all vibration exposure conditions of Experiment 1.

\begin{tabular}{|c|c|c|c|c|c|c|}
\hline \multirow[b]{2}{*}{ Percentile } & \multicolumn{2}{|c|}{ Low Target Force } & \multicolumn{2}{|c|}{ Med Target Force } & \multicolumn{2}{|c|}{ High Target Force } \\
\hline & Grip (15 N) & Push $(25 \mathrm{~N})$ & Grip (30 N) & Push $(50 \mathrm{~N})$ & Grip (45 N) & Push $(75 \mathrm{~N})$ \\
\hline 90th \%tile & 33.4 & 47.5 & 49.7 & 68.9 & 63.2 & 97.1 \\
\hline 75th \%tile & 26.2 & 39.0 & 40.7 & 60.9 & 54.1 & 84.4 \\
\hline Median & 21.1 & 32.1 & 31.4 & 51.5 & 44.1 & 73.2 \\
\hline 25 th $\%$ tile & 15.6 & 26.0 & 25.7 & 43.9 & 36.7 & 62.7 \\
\hline 10th \%tile & 12.3 & 21.9 & 21.7 & 38.4 & 30.7 & 55.9 \\
\hline
\end{tabular}



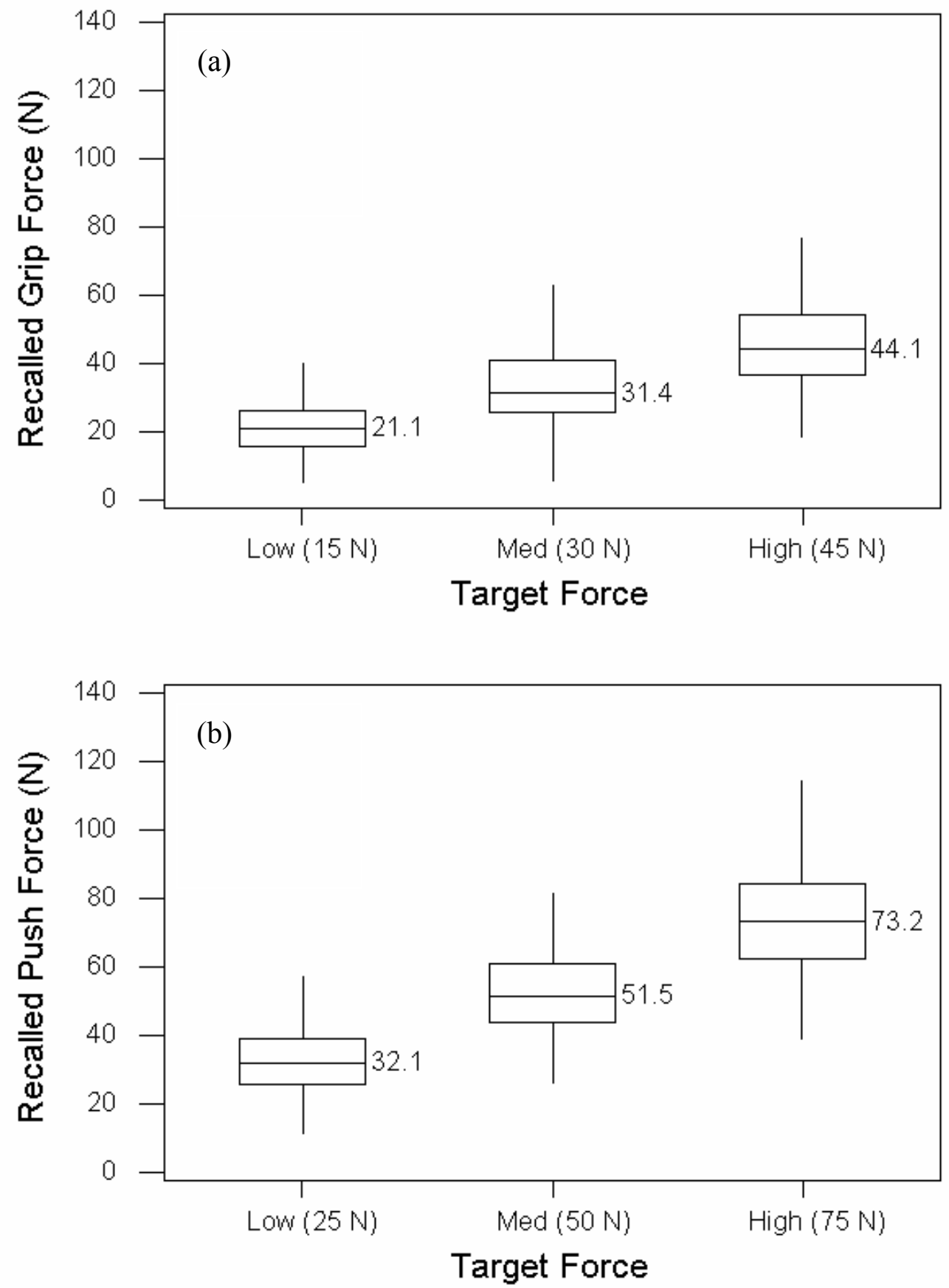

Figure 4.1.1 Distribution of recalled forces for (a) grip and (b) push by target force across all vibration exposure conditions of Experiment 1. 


\subsubsection{Analysis of variance (ANOVA) for Experiment 1}

Table 4.1.3 presents the results of the full factorial ANOVA findings for Experiment 1. As indicated in the table, frequency and force level were found to be significant main effects at the $\mathrm{p}<0.05$ level.

Table 4.1.3 ANOVA of grip and push force-recall error in the Experiment 1.

\begin{tabular}{|c|c|c|c|c|c|}
\hline SOURCE & $\mathrm{SS}$ & $\mathrm{DF}$ & $\mathrm{MS}$ & $\mathrm{F}$ & $\mathrm{p}<$ \\
\hline Vibration Frequency (Hz) & 8948.284 & 4 & 2237.071 & 16.301 & 0.001 \\
\hline Force Level (F) & 6901.501 & 2 & 3450.750 & 25.145 & 0.001 \\
\hline Rest Period (R) & 500.085 & 1 & 500.085 & 3.644 & 0.056 \\
\hline Day (D) & 66.650 & 1 & 66.650 & 0.486 & 0.486 \\
\hline Type of Exertion (T) & 2.601 & 1 & 2.601 & 0.019 & 0.891 \\
\hline $\mathrm{T} * \mathrm{R}$ & 948.027 & 1 & 948.027 & 6.908 & 0.009 \\
\hline $\mathrm{F} * \mathrm{R}$ & 603.446 & 2 & 301.723 & 2.199 & 0.111 \\
\hline $\mathrm{D} * \mathrm{~T}$ & 313.227 & 1 & 313.227 & 2.282 & 0.131 \\
\hline $\mathrm{T} * \mathrm{~F}$ & 525.128 & 2 & 262.564 & 1.913 & 0.148 \\
\hline $\mathrm{D} * \mathrm{~F}$ & 479.673 & 2 & 239.836 & 1.748 & 0.175 \\
\hline $\mathrm{F}^{*} \mathrm{~Hz}$ & 979.134 & 8 & 122.392 & 0.892 & 0.522 \\
\hline $\mathrm{D} * \mathrm{R}$ & 20.306 & 1 & 20.306 & 0.148 & 0.701 \\
\hline $\mathrm{D} * \mathrm{~Hz}$ & 255.097 & 4 & 63.774 & 0.465 & 0.762 \\
\hline $\mathrm{Hz} * \mathrm{R}$ & 216.330 & 4 & 54.082 & 0.394 & 0.813 \\
\hline $\mathrm{T} * \mathrm{~Hz}$ & 196.120 & 4 & 49.030 & 0.357 & 0.839 \\
\hline $\mathrm{T} * \mathrm{~F} * \mathrm{R}$ & 204.298 & 2 & 102.149 & 0.744 & 0.475 \\
\hline $\mathrm{F} * \mathrm{~Hz} * \mathrm{R}$ & 1008.677 & 8 & 126.085 & 0.919 & 0.500 \\
\hline $\mathrm{T} * \mathrm{~Hz} * \mathrm{R}$ & 390.916 & 4 & 97.729 & 0.712 & 0.584 \\
\hline $\mathrm{D} * \mathrm{~Hz} * \mathrm{R}$ & 282.651 & 4 & 70.663 & 0.515 & 0.725 \\
\hline $\mathrm{T} * \mathrm{~F} * \mathrm{~Hz}$ & 726.271 & 8 & 90.784 & 0.662 & 0.726 \\
\hline $\mathrm{D} * \mathrm{~T} * \mathrm{~F}$ & 58.401 & 2 & 29.201 & 0.213 & 0.808 \\
\hline $\mathrm{D} * \mathrm{~T} * \mathrm{R}$ & 3.099 & 1 & 3.099 & 0.023 & 0.881 \\
\hline $\mathrm{D} * \mathrm{~F} * \mathrm{R}$ & 24.702 & 2 & 12.351 & 0.090 & 0.914 \\
\hline $\mathrm{D} * \mathrm{~F} * \mathrm{~Hz}$ & 408.023 & 8 & 51.003 & 0.372 & 0.936 \\
\hline $\mathrm{D} * \mathrm{~T} * \mathrm{~Hz}$ & 92.211 & 4 & 23.053 & 0.168 & 0.955 \\
\hline $\mathrm{D} * \mathrm{~T} * \mathrm{~F} * \mathrm{R}$ & 184.931 & 2 & 92.465 & 0.674 & 0.510 \\
\hline $\mathrm{D} * \mathrm{~F} * \mathrm{~Hz} * \mathrm{R}$ & 714.012 & 8 & 89.252 & 0.650 & 0.736 \\
\hline $\mathrm{D} * \mathrm{~T} * \mathrm{~Hz} * \mathrm{R}$ & 222.622 & 4 & 55.655 & 0.406 & 0.805 \\
\hline $\mathrm{T} * \mathrm{~F} * \mathrm{~Hz} * \mathrm{R}$ & 593.286 & 8 & 74.161 & 0.540 & 0.827 \\
\hline $\mathrm{D} * \mathrm{~T} * \mathrm{~F} * \mathrm{~Hz}$ & 519.949 & 8 & 64.994 & 0.474 & 0.875 \\
\hline $\mathrm{D} * \mathrm{~T} * \mathrm{~F} * \mathrm{~Hz} * \mathrm{R}$ & 313.680 & 8 & 39.210 & 0.286 & 0.971 \\
\hline Error & 181147.003 & 1320 & 137.233 & & \\
\hline Total & 246773.380 & 1440 & & & \\
\hline Corrected Total & 207850.339 & 1439 & & & \\
\hline
\end{tabular}


Rest period duration was suggestively significant. Only one second-order interaction (Type of Exertion by Rest Period) was statistically significant at the 0.05 level. No higher-order interactions were significant or suggestively so.

\subsubsection{Statistical power for Experiment 1}

Equations 3-2 and 3-3 were used to evaluate the statistical power of Experiment 1 for main effects and two-way interactions, respectively. The "worst case" or lowest power is that associated with the main effect with the most levels. In this experiment, the worst case for testing main effects is represented by vibration frequency (5 levels). The variance of the response variable (force recall error) was found to be $137 \mathrm{~N}^{2}$. The results of applying Equation 3-2 to the data collected in Experiment 1 are contained in Table 4.1.4.

Table 4.1.4 Sensitivity of Experiment 1 for detecting force-recall error differences due to vibration frequency effects at three levels of type II error probability $(\beta)$.

\begin{tabular}{|c|c|c|c|c|c|c|c|c|c|c|}
\hline $\begin{array}{l}\text { Sensitivity } \\
\text { (Newtons) }\end{array}$ & $\begin{array}{c}\text { Parameter } \\
\text { value } \\
\text { (from table) }\end{array}$ & $\begin{array}{c}\text { Levels of } \\
\text { factor a }\end{array}$ & $\begin{array}{l}\text { Observed } \\
\text { variance }\end{array}$ & $\begin{array}{c}\text { Number } \\
\text { of } \\
\text { subjects }\end{array}$ & $\begin{array}{c}\text { Trials at } \\
\text { each } \\
\text { level }\end{array}$ & $\begin{array}{c}\text { Replicates } \\
\text { at each } \\
\text { level }\end{array}$ & $\begin{array}{c}\text { Numerator } \\
\text { DF } \\
(a-1)\end{array}$ & $\begin{array}{c}\text { Denominator } \\
\text { DF } \\
a(n-1)\end{array}$ & $\begin{array}{c}\text { Type II } \\
\text { error }\end{array}$ & $\begin{array}{l}\text { Type I } \\
\text { error }\end{array}$ \\
\hline D & $\Phi$ & $\mathbf{a}$ & $\sigma^{2}$ & & & n & $v_{1}$ & $v_{2}$ & $1-\beta$ & $1-\alpha$ \\
\hline 5.2 & 1.7 & 5 & 137 & 12 & 12 & 144 & 4 & 715 & 0.90 & 0.95 \\
\hline 4.6 & 1.5 & 5 & 137 & 12 & 12 & 144 & 4 & 715 & 0.80 & 0.95 \\
\hline 4.0 & 1.3 & 5 & 137 & 12 & 12 & 144 & 4 & 715 & 0.70 & 0.95 \\
\hline
\end{tabular}


As indicated in the table, with this experiment's observed variance and sample size, the statistical test for evaluating this main effect demonstrated a sensitivity of $5.2 \mathrm{~N}$ with a power of $0.90,4.6 \mathrm{~N}$ with a power of 0.80 , and $4.0 \mathrm{~N}$ with a power of 0.70 .

In Experiment 1, the worst case for testing two-way interactions is represented by the vibration frequency (5 levels) by force level (3 levels) interaction. The results of applying Equation 3-3 to the data collected in Experiment 1 are contained in Table 4.1.5. As indicated in this table, with this experiment's observed variance and sample size, the statistical test for evaluating this interaction effect demonstrated a sensitivity of $10.8 \mathrm{~N}$ with a power of $0.90,9.3 \mathrm{~N}$ with a power of 0.80 , and $8.6 \mathrm{~N}$ with a power of 0.70 .

Table 4.1.5 Sensitivity of Experiment 1 for detecting force-recall error differences due to the vibration frequency by force level interaction effects at three levels of type II error probability $(\beta)$.

\begin{tabular}{|c|c|c|c|c|c|c|c|c|c|c|c|}
\hline $\begin{array}{l}\text { Sensitivity } \\
\text { (Newtons) }\end{array}$ & $\begin{array}{c}\text { Parameter } \\
\text { value } \\
\text { (from table) }\end{array}$ & $\begin{array}{c}\text { Levels } \\
\text { of factor } \\
a\end{array}$ & $\begin{array}{c}\text { Levels } \\
\text { of factor } \\
b\end{array}$ & $\begin{array}{l}\text { Observed } \\
\text { variance }\end{array}$ & $\begin{array}{c}\text { Number } \\
\text { of } \\
\text { subjects }\end{array}$ & $\begin{array}{c}\text { Trials } \\
\text { at each } \\
\text { level }\end{array}$ & $\begin{array}{c}\text { Replicates } \\
\text { at each } \\
\text { level }\end{array}$ & $\begin{array}{c}\text { Numerator } \\
\text { DF } \\
(a-1)(b-1)\end{array}$ & $\begin{array}{c}\text { Denominator } \\
\text { DF } \\
a b(n-1)\end{array}$ & $\begin{array}{l}\text { Type II } \\
\text { error }\end{array}$ & $\begin{array}{l}\text { Type I } \\
\text { error }\end{array}$ \\
\hline D & $\Phi$ & $\mathbf{a}$ & b & $\sigma^{2}$ & & & n & $v_{1}$ & $v_{2}$ & $1-\beta$ & $1-\alpha$ \\
\hline 10.8 & 1.5 & 5 & 3 & 137 & 12 & 4 & 48 & 8 & 705 & 0.90 & 0.95 \\
\hline 9.3 & 1.3 & 5 & 3 & 137 & 12 & 4 & 48 & 8 & 705 & 0.80 & 0.95 \\
\hline 8.6 & 1.2 & 5 & 3 & 137 & 12 & 4 & 48 & 8 & 705 & 0.70 & 0.95 \\
\hline
\end{tabular}




\subsubsection{Vibration frequency effects in Experiment 1}

Table 4.1.6 contains the means, standard deviations, and 95\% confidence intervals for recall errors for each frequency of vibration exposure employed in Experiment 1.

Table 4.1.6 Force-recall error (in Newtons) at each vibration frequency in Experiment 1.

\begin{tabular}{|c|c|c|c|c|}
\hline \multirow{2}{*}{$\begin{array}{c}\text { Vibration } \\
\text { Freq in } \mathrm{Hz}\end{array}$} & \multirow{2}{*}{ Mean } & \multirow{2}{*}{ SD } & \multicolumn{2}{|c|}{ 95\% Confidence Interval } \\
\hline & & & Lower Bound & Upper Bound \\
\hline 0.0 & 2.7 & 10.1 & 1.5 & 3.9 \\
\hline 12.5 & 2.3 & 11.5 & 0.9 & 3.6 \\
\hline 40.0 & 8.6 & 12.9 & 7.1 & 10.1 \\
\hline 125.0 & 7.4 & 12.4 & 6.0 & 8.8 \\
\hline 250.0 & 5.0 & 11.1 & 3.7 & 6.4 \\
\hline
\end{tabular}

As depicted in Figures 4.1.2 through 4.1.5, vibration frequency effects were curvilinear in nature with greatest errors produced at $40 \mathrm{~Hz}$. The graphics also indicates that vibration frequency affected grip and push efforts in a similar fashion. 


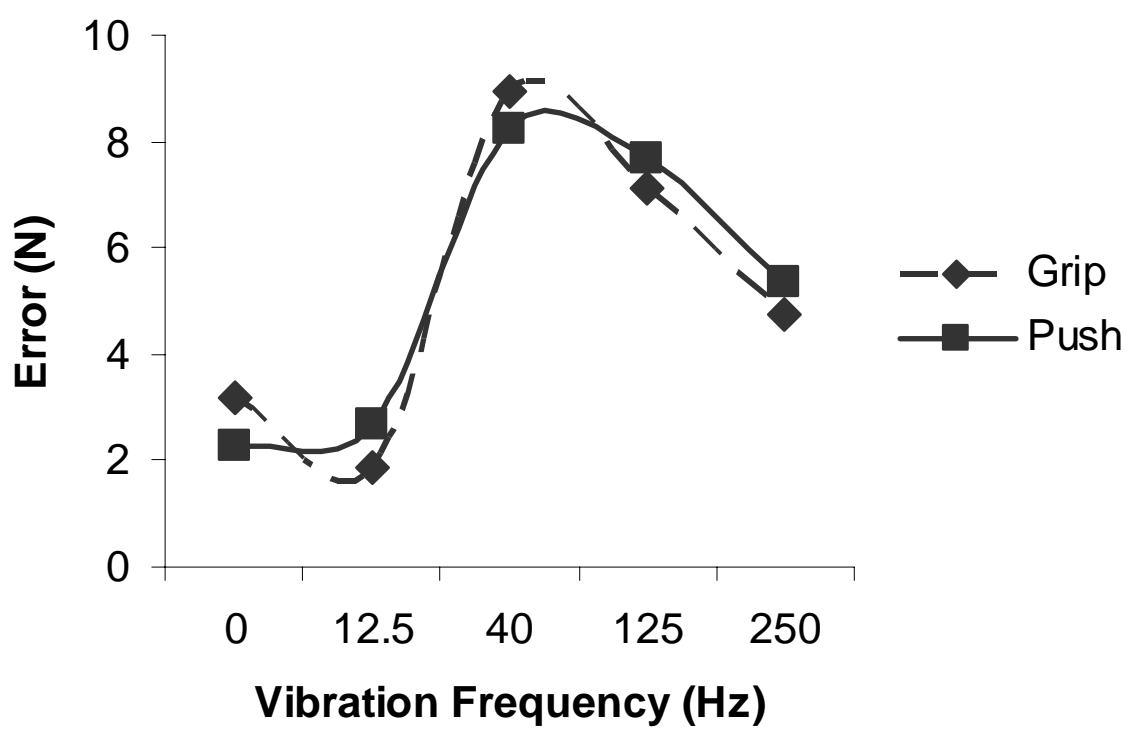

Figure 4.1.2 Experiment 1 grip and push force-recall error means plotted against vibration frequency across all force levels.

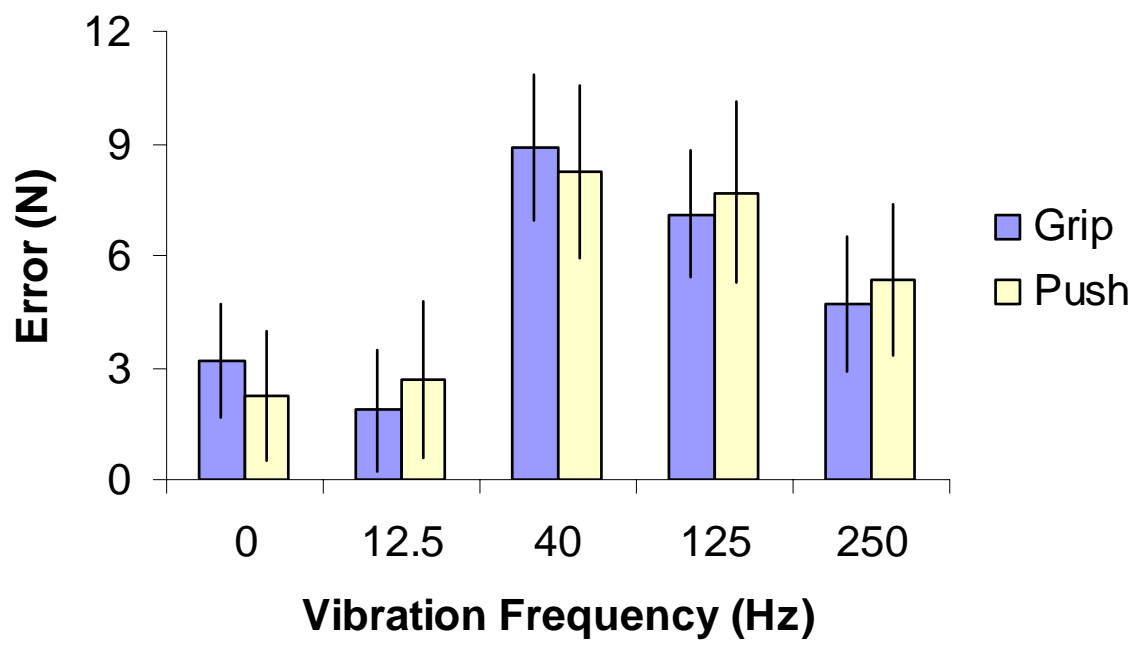

Figure 4.1.3 Experiment 1 grip and push force-recall error means plotted against vibration frequency. (Y error bars $=95 \%$ C.I.) 


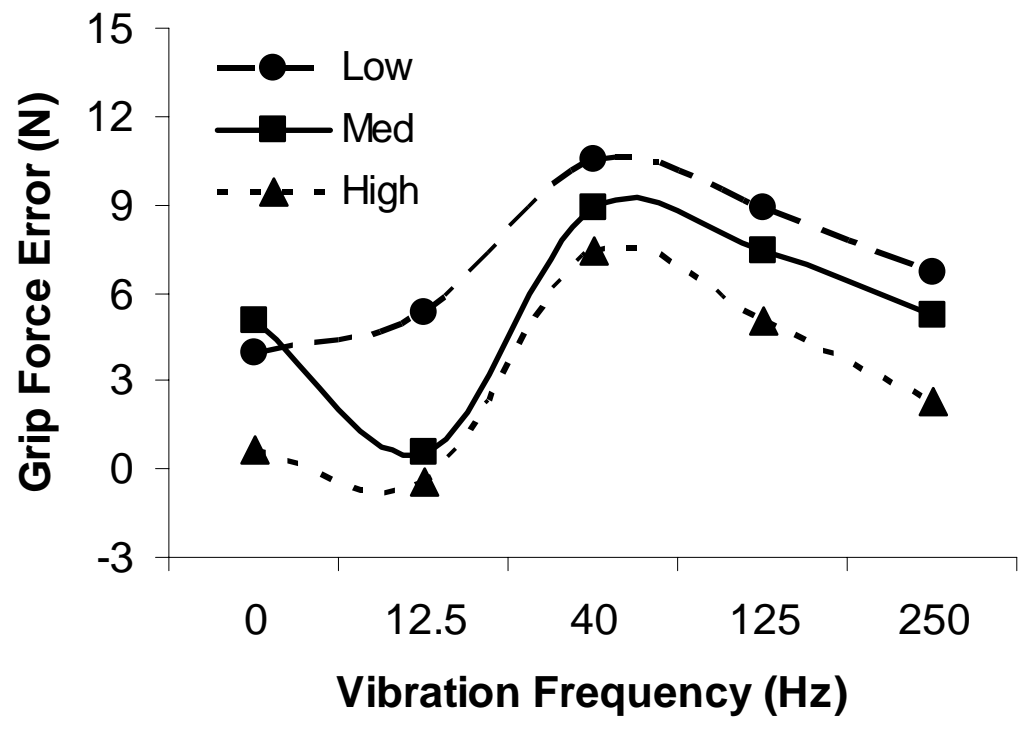

Figure 4.1.4 Experiment 1 grip force-recall error means for low, medium, and high target force levels plotted against vibration frequency.

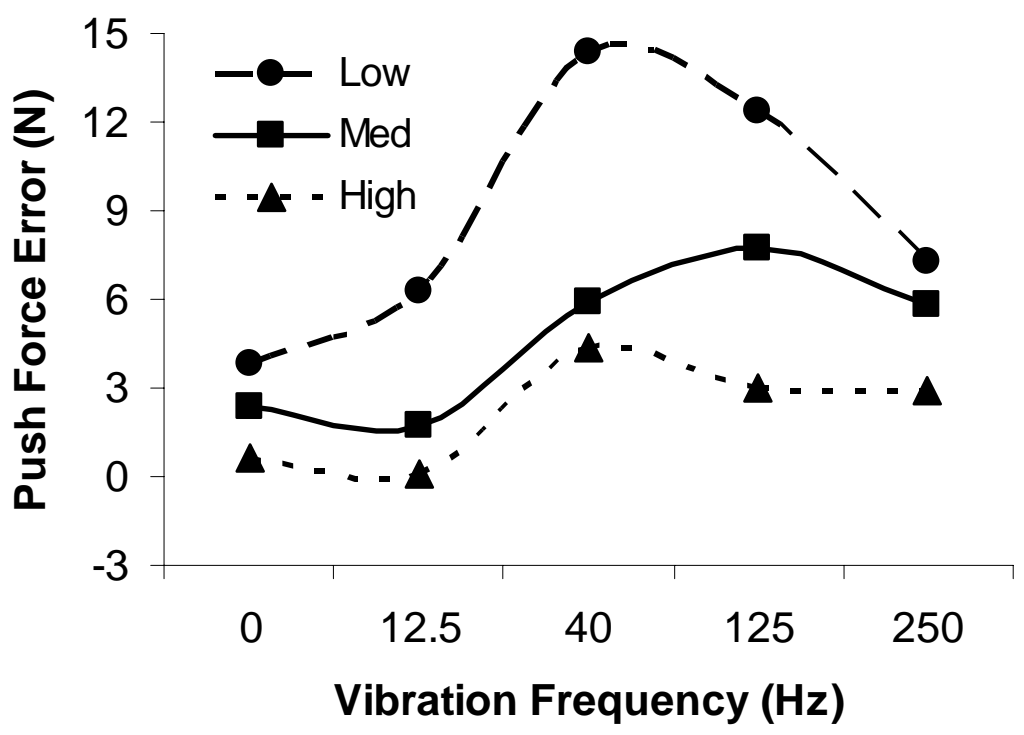

Figure 4.1.5 Experiment 1 push force-recall error means for low, medium, and high target force levels plotted against vibration frequency. 
Pairwise comparisons using Tukey's Honestly Significant Difference (HSD) procedure are contained in Table 4.1.7. The results showed that the mean of force-recall errors at $40 \mathrm{~Hz}$ was significantly different than the means at $0 \mathrm{~Hz}, 12.5 \mathrm{~Hz}$, and $250 \mathrm{~Hz}$ at the $\mathrm{p}<0.05$ level, but was not different than mean error at $125 \mathrm{~Hz}$. Mean error for $125 \mathrm{~Hz}$ was significantly different than the $0 \mathrm{~Hz}$ and $12.5 \mathrm{~Hz}$ error means, but was not different than the error mean at $250 \mathrm{~Hz}$. The $12.5-\mathrm{Hz}$ and $250-\mathrm{Hz}$ error means were not significantly different than the zero vibration condition.

Table 4.1.7 Tukey's HSD pair-wise recall error mean comparisons for vibration frequency in Experiment 1.

\begin{tabular}{|c|c|c|c|c|c|c|}
\hline \multirow{2}{*}{$\begin{array}{l}\text { (I) } \\
\text { Freq. } \\
(\mathrm{Hz}) .\end{array}$} & \multirow{2}{*}{$\begin{array}{l}\text { (J) } \\
\text { Freq. } \\
(\mathrm{Hz}) .\end{array}$} & \multirow{2}{*}{$\begin{array}{c}\text { Mean } \\
\text { Difference } \\
(\mathrm{I}-\mathrm{J})\end{array}$} & \multirow[b]{2}{*}{ Std. Error } & \multirow[b]{2}{*}{ Sig. } & \multicolumn{2}{|c|}{$\begin{array}{c}95 \% \text { Confidence Interval for } \\
\text { Difference }\end{array}$} \\
\hline & & & & & Lower Bound & Upper Bound \\
\hline \multirow[t]{4}{*}{0.0} & 12.5 & 0.5 & 0.98 & 0.991 & -2.2 & 3.1 \\
\hline & 40.0 & -5.9 & 0.98 & 0.001 & -8.5 & -3.2 \\
\hline & 125.0 & -4.7 & 0.98 & 0.001 & -7.3 & -2.0 \\
\hline & 250.0 & -2.3 & 0.98 & 0.122 & -5.0 & 0.3 \\
\hline \multirow[t]{4}{*}{12.5} & 0.0 & -0.5 & 0.98 & 0.991 & -3.2 & 2.2 \\
\hline & 40.0 & -6.3 & 0.98 & 0.001 & -9.0 & -3.7 \\
\hline & 125.0 & -5.1 & 0.98 & 0.001 & -7.8 & -2.5 \\
\hline & 250.0 & -2.8 & 0.98 & $\mathbf{0 . 0 3 7}$ & -5.4 & -0.1 \\
\hline \multirow[t]{4}{*}{40.0} & 0.0 & 5.9 & 0.98 & 0.001 & 3.2 & 8.5 \\
\hline & 12.5 & 6.3 & 0.98 & 0.001 & 3.7 & 9.0 \\
\hline & 125.0 & 1.2 & 0.98 & 0.744 & -1.5 & 3.9 \\
\hline & 250.0 & 3.5 & 0.98 & 0.003 & 0.9 & 6.2 \\
\hline \multirow[t]{4}{*}{125.0} & 0.0 & 4.7 & 0.98 & 0.001 & 2.0 & 7.3 \\
\hline & 12.5 & 5.1 & 0.98 & 0.001 & 2.5 & 7.8 \\
\hline & 40.0 & -1.2 & 0.98 & 0.744 & -3.9 & 1.5 \\
\hline & 250.0 & 2.4 & 0.98 & 0.111 & -0.3 & 5.0 \\
\hline \multirow[t]{4}{*}{250.0} & 0.0 & 2.3 & 0.98 & 0.122 & -0.3 & 5.0 \\
\hline & 12.5 & 2.8 & 0.98 & $\mathbf{0 . 0 3 7}$ & 0.1 & 5.4 \\
\hline & 40.0 & -3.5 & 0.98 & 0.003 & -6.2 & -0.9 \\
\hline & 125.0 & -2.4 & 0.98 & 0.111 & -5.0 & 0.3 \\
\hline
\end{tabular}




\subsubsection{Force level effects in Experiment 1}

Table 4.1.8, Figure 4.1.6, and Figure 4.1.7 demonstrate the relationship between force level and force-recall error means in Experiment 1. As shown in the figure, accuracy of grip and push force recall improved as force level increased. Tukey's HSD pairwise comparisons revealed significant differences between all force levels $(\mathrm{p}<0.05)$.

Table 4.1.8 Force-recall error (in Newtons) at each level of hand-handle coupling force in Experiment 1.

\begin{tabular}{ccccc}
\hline \multirow{2}{*}{ Force Level } & \multirow{2}{*}{ Mean } & \multirow{2}{*}{ SD } & \multicolumn{2}{c}{$95 \%$ Confidence Interval } \\
\cline { 4 - 5 } & & & Lower Bound & Upper Bound \\
\hline Low & 7.9 & 9.4 & 7.1 & 8.8 \\
Med & 5.1 & 11.8 & 4.0 & 6.1 \\
High & 2.6 & 13.9 & 1.3 & 3.8 \\
\hline
\end{tabular}

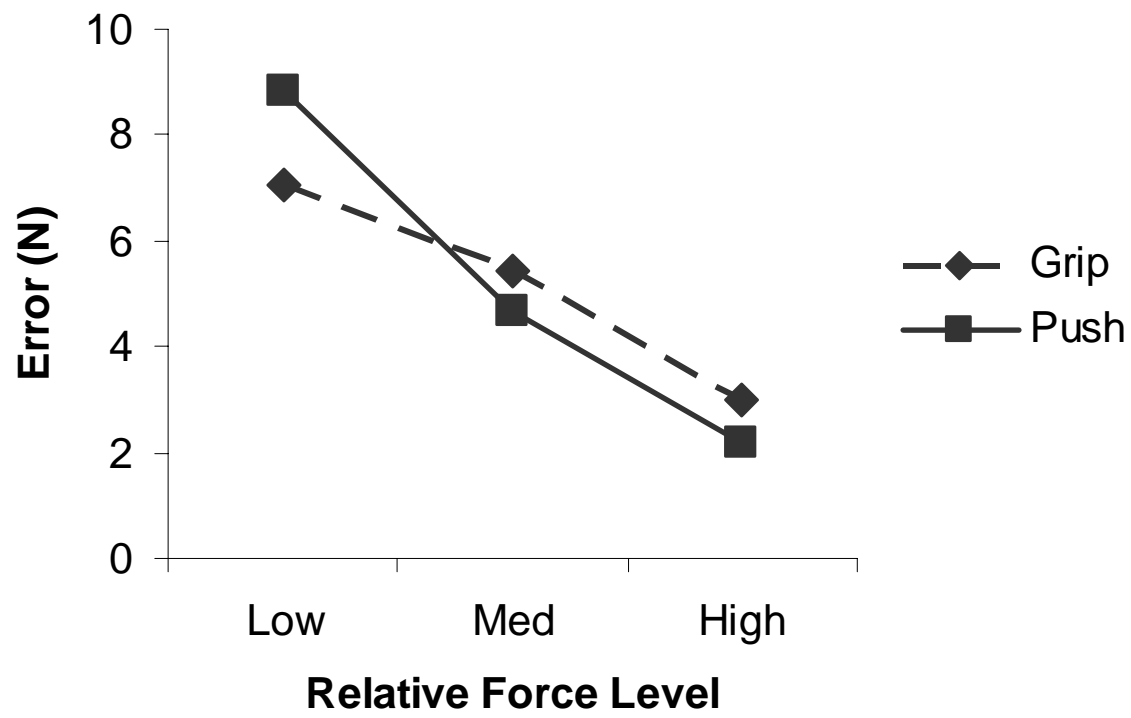

Figure 4.1.6 Force-recall error versus relative grip and push force levels across all frequencies of vibration in Experiment 1. 


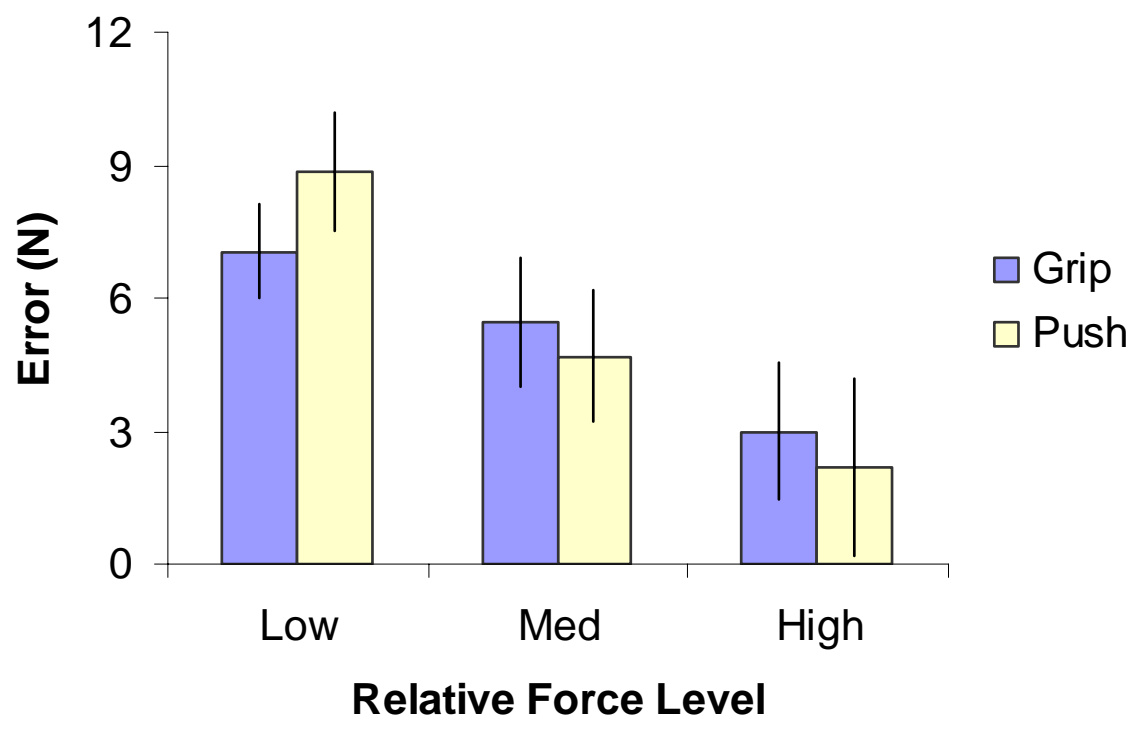

Figure 4.1.7 Force-recall error versus relative grip and push force levels in Experiment 1. (Y error bars $=95 \%$ C.I.)

\subsubsection{Test-retest reliability in Experiment 1}

A comparison of the force-recall errors for Day 1 and Day 2 is contained in Table 4.1.9. The ANOVA revealed no difference in recall error means between test days.

Table 4.1.9 Force-recall error (in Newtons) for each test session in Experiment 1.

\begin{tabular}{ccccc}
\hline \multirow{2}{*}{ Day } & \multirow{2}{*}{ Mean } & \multirow{2}{*}{ SD } & \multicolumn{2}{c}{$95 \%$ Confidence Interval } \\
\cline { 4 - 5 } & & & Lower Bound & Upper Bound \\
\hline Day 1 & 5.4 & 12.9 & 4.5 & 6.4 \\
Day 2 & 5.0 & 11.1 & 4.2 & 5.8 \\
\hline
\end{tabular}


To further examine test-retest reliability, the actual grip and push recall forces produced in Day 1 were compared with those of Day 2. The Pearson product-moment correlation results indicated strong test-retest reliability as correlations for each subject were found to be significant for both grip and push recall forces.

Table 4.1.10 Test-retest correlations for recalled grip and push forces for each subject across all conditions for Experiment 1.

\begin{tabular}{cll}
\hline Subject & Grip & Push \\
\hline 1 & $0.63^{*}$ & $0.76^{*}$ \\
2 & $0.74^{*}$ & $0.90^{*}$ \\
3 & $0.69^{*}$ & $0.75^{*}$ \\
4 & $0.55^{*}$ & $0.85^{*}$ \\
5 & $0.65^{*}$ & $0.82^{*}$ \\
6 & $0.66^{*}$ & $0.87^{*}$ \\
7 & $0.78^{*}$ & $0.88^{*}$ \\
8 & $0.66^{*}$ & $0.74^{*}$ \\
9 & $0.88^{*}$ & $0.94^{*}$ \\
10 & $0.65^{*}$ & $0.77^{*}$ \\
11 & $0.71^{*}$ & $0.75^{*}$ \\
12 & $0.54^{*}$ & $0.84^{*}$ \\
\hline Mean & $0.68^{*}$ & $0.82^{*}$ \\
Min & $0.54^{*}$ & $0.74^{*}$ \\
Max & $0.88^{*}$ & $0.94^{*}$ \\
\hline
\end{tabular}

*significant at 0.01 level (2-tailed)

\subsubsection{Exertion type effects in Experiment 1}

The ANOVA revealed no differences between the means of recalled grip error and recalled push error . The means, standard deviations, and 95\% confidence intervals for each exertion type are contained in Table 4.1.11. 
Table 4.1.11 Grip and push force-recall error means (in Newtons) across all conditions of Experiment 1.

\begin{tabular}{ccccc}
\hline $\begin{array}{c}\text { Exertion } \\
\text { Type }\end{array}$ & Mean & SD & \multicolumn{2}{c}{$95 \%$ Confidence Interval } \\
\cline { 4 - 5 } & & & Lower Bound & Upper Bound \\
\hline Grip & 5.2 & 10.8 & 4.4 & 5.9 \\
Push & 5.2 & 13.1 & 4.3 & 6.2 \\
\hline
\end{tabular}

\subsubsection{Effects of rest period duration in Experiment 1}

Overall, grip and push force-recall errors were statistically equivalent without regard to the duration of the rest period between force production/memorization and force-recall periods. However, this difference was suggestively significant. Grip force-recall errors were statistically equivalent regardless of the time period between the vibration exposure and the recall period. However, as indicated in Table 4.1.13 and shown in Figure 4.1.8 and Figure 4.1.9, errors in push force-recall declined when the rest period increased from $10 \mathrm{~s}$ to $20 \mathrm{~s}$.

Table 4.1.12 Force-recall error means (in Newtons) at each rest period duration evaluated in Experiment 1.

\begin{tabular}{|c|c|c|c|c|}
\hline \multirow{2}{*}{ Rest Period } & \multirow{2}{*}{ Mean } & \multirow{2}{*}{ SD } & \multicolumn{2}{|c|}{ 95\% Confidence Interval } \\
\hline & & & Lower Bound & Upper Bound \\
\hline $10 \mathrm{~s}$ & 5.8 & 12.0 & 4.9 & 6.7 \\
\hline $20 \mathrm{~s}$ & 4.6 & 12.1 & 3.7 & 5.5 \\
\hline
\end{tabular}


Table 4.1.13 Force-recall error means (in Newtons) for each exertion type and rest period duration evaluated in Experiment 1.

\begin{tabular}{cccccc}
\hline $\begin{array}{c}\text { Exertion } \\
\text { Type }\end{array}$ & Rest Period & Mean & SD & \multicolumn{2}{c}{ 95\% Confidence Interval } \\
\hline \multirow{2}{*}{ Grip } & $10 \mathrm{~s}$ & 4.9 & 10.9 & Lower Bound Upper Bound \\
& $20 \mathrm{~s}$ & 5.4 & 10.8 & 4.7 & 6.1 \\
\multirow{2}{*}{ Push } & $10 \mathrm{~s}$ & 6.6 & 12.9 & 5.4 & 7.9 \\
& $20 \mathrm{~s}$ & 3.8 & 13.2 & 2.6 & 5.1 \\
\hline
\end{tabular}

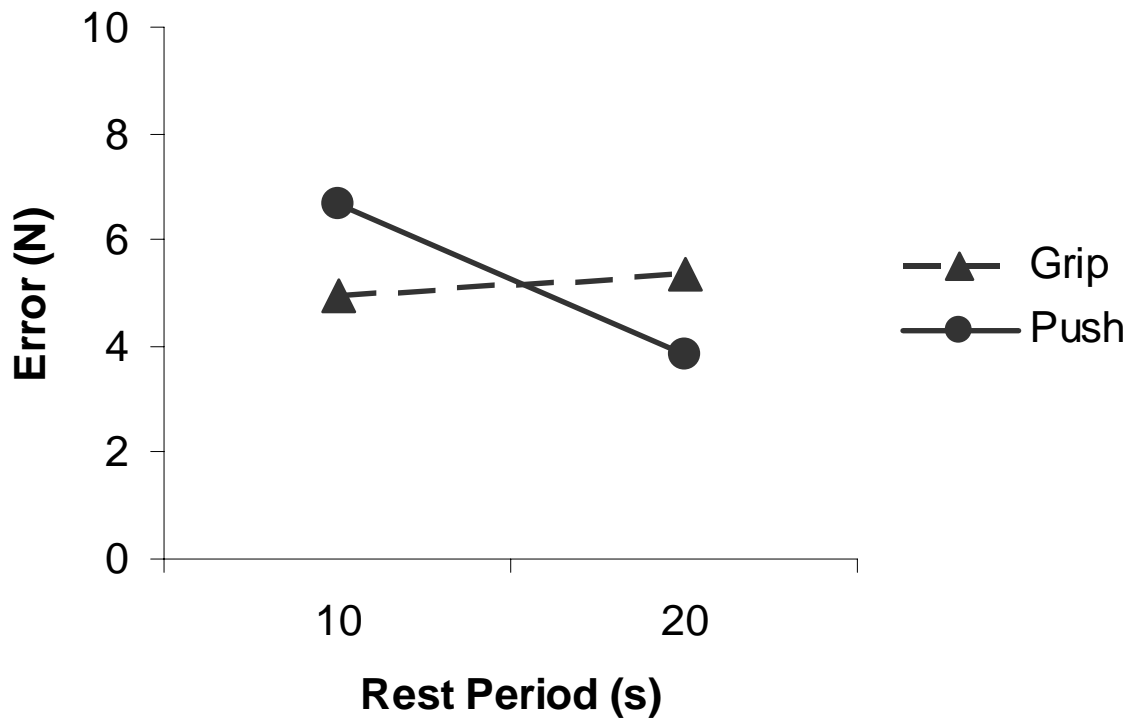

Figure 4.1.8 Force-recall error as a function of exertion type and rest period duration in Experiment 1. 


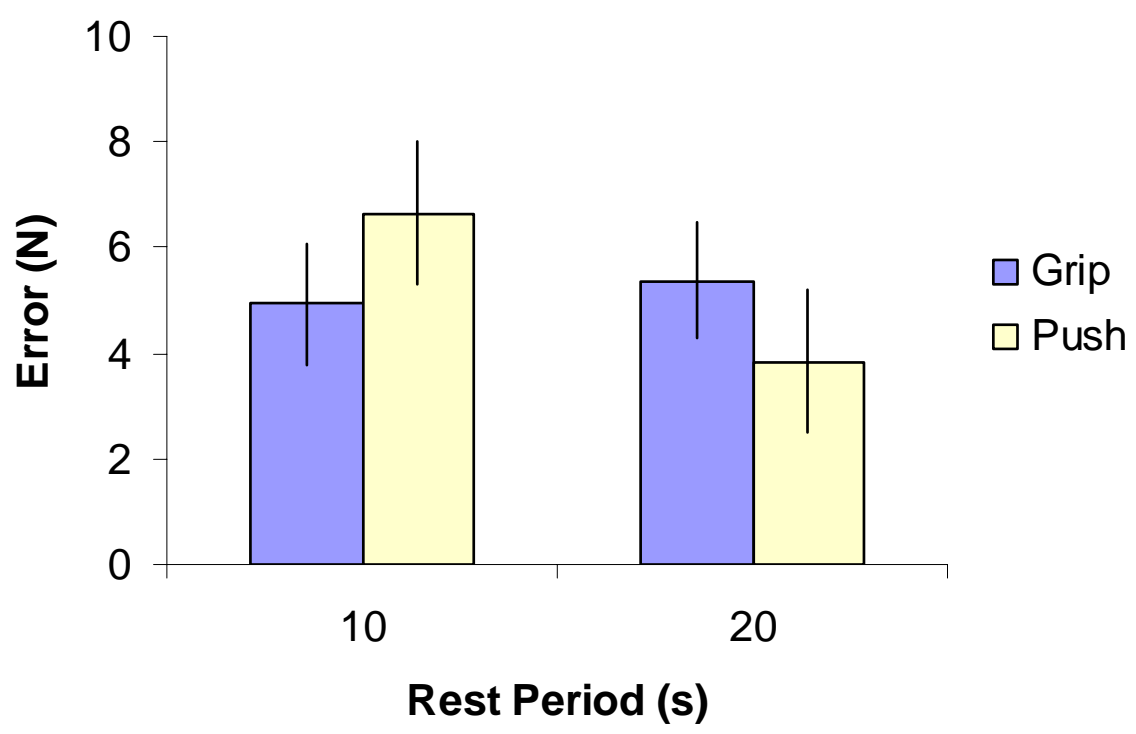

Figure 4.1.9 Force-recall error as a function of exertion type and rest period duration in Experiment 1. (Y error bars $=95 \%$ C.I.) 


\subsection{Experiment 2 results}

\subsubsection{Subject descriptors for Experiment 2}

Table 4.2.1 contains descriptors for the 20 subjects ( 10 female and 10 male) who participated in Experiment 2.

Table 4.2.1 Descriptors of the twenty participants of Experiment 2.

\begin{tabular}{|c|c|c|c|c|c|c|c|c|}
\hline Subject & $\begin{array}{l}\text { Age } \\
\text { (yrs) }\end{array}$ & $\begin{array}{c}\text { Stature } \\
(\mathrm{m})\end{array}$ & $\begin{array}{c}\text { Weight } \\
(\mathrm{kg})\end{array}$ & $\begin{array}{l}{ }^{1} \text { Hand } \\
\text { breadth } \\
(\mathrm{mm})\end{array}$ & $\begin{array}{l}{ }^{2} \text { Hand } \\
\text { length } \\
(\mathrm{mm})\end{array}$ & $\begin{array}{c}{ }^{3} \text { Hand } \\
\text { circum. } \\
(\mathrm{mm})\end{array}$ & $\begin{array}{c}{ }^{4} \text { Hand } \\
\text { volume } \\
(\mathrm{ml})\end{array}$ & $\begin{array}{c}{ }^{5} \text { Forearm } \\
\text { volume } \\
(\mathrm{ml})\end{array}$ \\
\hline $\mathrm{F} 1$ & 18 & 1.70 & 63.2 & 74 & 174 & 178 & 245 & 965 \\
\hline $\mathrm{F} 2$ & 33 & 1.73 & 67.0 & 83 & 184 & 198 & 285 & 1000 \\
\hline F3 & 26 & 1.61 & 57.2 & 82 & 177 & 195 & 260 & 940 \\
\hline $\mathrm{F} 4$ & 27 & 1.63 & 52.0 & 73 & 180 & 183 & 250 & 910 \\
\hline F5 & 22 & 1.57 & 64.0 & 76 & 171 & 187 & 258 & 1095 \\
\hline F6 & 45 & 1.65 & 71.8 & 76 & 178 & 187 & 275 & 1200 \\
\hline F7 & 27 & 1.68 & 62.0 & 75 & 170 & 180 & 263 & 1075 \\
\hline F8 & 35 & 1.52 & 46.4 & 70 & 158 & 171 & 195 & 611 \\
\hline F9 & 24 & 1.61 & 54.4 & 71 & 176 & 178 & 235 & 950 \\
\hline F10 & 29 & 1.63 & 75.2 & 74 & 181 & 180 & 313 & 1205 \\
\hline M1 & 28 & 1.75 & 75.4 & 87 & 181 & 210 & 313 & 1700 \\
\hline M2 & 22 & 1.78 & 81.6 & 94 & 188 & 221 & 400 & 1675 \\
\hline M3 & 27 & 1.88 & 90.8 & 88 & 198 & 207 & 380 & 1675 \\
\hline M4 & 29 & 1.78 & 72.6 & 90 & 192 & 202 & 383 & 1450 \\
\hline M5 & 33 & 1.78 & 65.9 & 85 & 183 & 208 & 370 & 1490 \\
\hline M6 & 28 & 1.78 & 87.4 & 88 & 193 & 212 & 428 & 1700 \\
\hline M7 & 27 & 1.91 & 112.8 & 80 & 188 & 200 & 345 & 1570 \\
\hline M8 & 21 & 1.73 & 102.2 & 89 & 186 & 223 & 432 & 1800 \\
\hline M9 & 19 & 1.98 & 102.2 & 100 & 203 & 238 & 500 & 1980 \\
\hline M10 & 19 & 1.73 & 63.5 & 87 & 184 & 213 & 350 & 1340 \\
\hline Mean & 27 & 1.72 & 73.4 & 82 & 182 & 199 & 324 & 1317 \\
\hline Min & 18 & 1.52 & 46.4 & 70 & 158 & 171 & 195 & 611 \\
\hline Max & 45 & 1.98 & 112.8 & 100 & 203 & 238 & 500 & 1980 \\
\hline $\mathrm{SD}$ & 6.4 & 0.11 & 17.9 & 8.3 & 10.3 & 18.0 & 80.0 & 372.3 \\
\hline $\begin{array}{l}1 \text { at metac } \\
2 \text { from tip } \\
3 \text { at metac }\end{array}$ & $\begin{array}{l}\text { arpals } \\
\text { of } 3 \mathrm{rd} \\
\text { arpals }\end{array}$ & ger & DSe & & & & & \\
\hline
\end{tabular}




\subsubsection{Recalled grip and push force distributions in Experiment 2}

Table 4.2.2 and Figure 4.2.1 provide information regarding the subjects' abilities to recall and reproduce the memorized grip and push forces in Experiment 2. The box and whisker plots shown in Figure 4.2.1 display the distribution of the subjects' grip and push force-recall attempts at each force level. The box represents the interquartile range of the responses while the upper and lower whiskers extend to the $90^{\text {th }}$ and $10^{\text {th }}$ percentiles, respectively. The median response value for each group is also indicated in the figure.

Table 4.2.2 Distribution of recalled grip and push forces (in Newtons) across all vibration exposure conditions of Experiment 2.

\begin{tabular}{|c|c|c|c|c|c|c|}
\hline \multirow[b]{2}{*}{ Percentile } & \multicolumn{2}{|c|}{ Low Target Force } & \multicolumn{2}{|c|}{ Med Target Force } & \multicolumn{2}{|c|}{ High Target Force } \\
\hline & Grip $(15 \mathrm{~N})$ & Push $(25 \mathrm{~N})$ & Grip (30 N) & Push $(50 \mathrm{~N})$ & Grip (45 N) & Push $(75 \mathrm{~N})$ \\
\hline 90th \%tile & 34.5 & 42.2 & 54.8 & 70.4 & 74.2 & 105.3 \\
\hline 75 th $\%$ tile & 25.4 & 35.6 & 42.3 & 61.9 & 60.9 & 91.1 \\
\hline Median & 19.4 & 29.5 & 32.6 & 53.1 & 46.8 & 77.2 \\
\hline 25 th $\%$ tile & 15.2 & 24.0 & 26.0 & 45.0 & 36.9 & 69.7 \\
\hline 10th \%tile & 12.0 & 18.7 & 21.2 & 38.3 & 30.7 & 61.5 \\
\hline
\end{tabular}



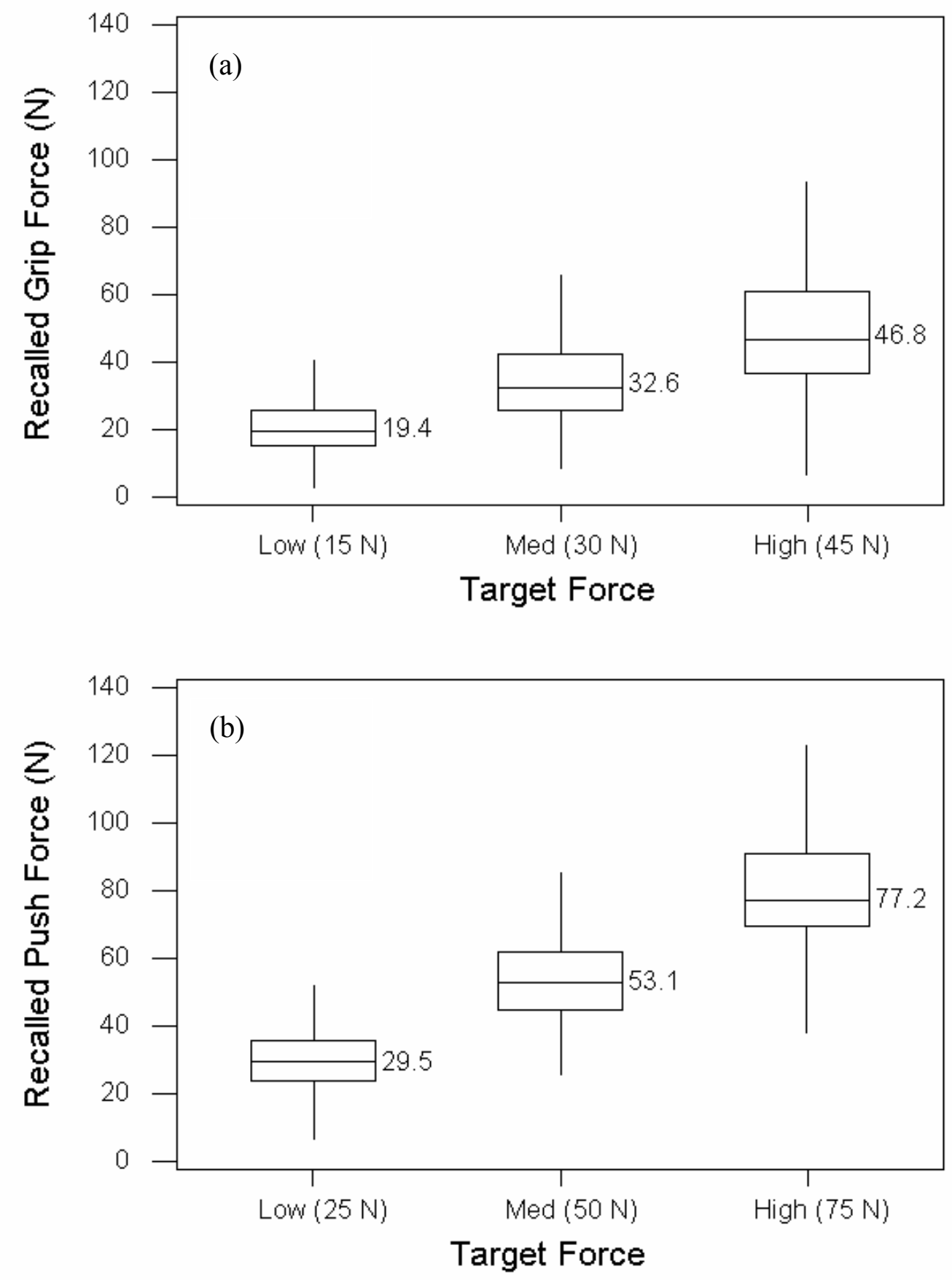

Figure 4.2.1 Distribution of recalled forces for (a) grip and (b) push by target force across all vibration exposure conditions of Experiment 2. 


\subsubsection{Analysis of variance (ANOVA) for Experiment 2}

Table 4.2.3 presents the results of the full factorial ANOVA for Experiment 2. As indicated in the table, vibration frequency, vibration magnitude, test day, and gender were found to be significant main effects at the $\mathrm{p}<0.05$ level. Force level was suggestively significant. Two two-way interactions (vibration magnitude by vibration frequency and exertion type by vibration magnitude) were statistically significant. One other two-way interaction (exertion type by gender) was suggestively significant. No higher-order interactions were significant. 
Table 4.2.3 ANOVA of grip and push force-recall error in Experiment 2.

\begin{tabular}{|c|c|c|c|c|c|}
\hline SOURCE & $S S$ & $D F$ & $M S$ & $F$ & $p<$ \\
\hline Vibration Frequency $(\mathrm{Hz})$ & 4500.897 & 3 & 1500.299 & 6.733 & 0.001 \\
\hline Vibration Magnitude (M) & 8716.813 & 1 & 8716.813 & 39.122 & 0.001 \\
\hline Gender (G) & 5067.761 & 1 & 5067.761 & 22.745 & 0.001 \\
\hline Day (D) & 1069.964 & 1 & 1069.964 & 4.802 & 0.029 \\
\hline Force Level (F) & 1099.482 & 2 & 549.741 & 2.467 & 0.085 \\
\hline Exertion Type (T) & 227.197 & 1 & 227.197 & 1.020 & 0.313 \\
\hline $\mathrm{M} * \mathrm{~Hz}$ & 3033.782 & 3 & 1011.261 & 4.539 & 0.004 \\
\hline $\mathrm{M} * \mathrm{~T}$ & 1341.011 & 1 & 1341.011 & 6.019 & 0.014 \\
\hline $\mathrm{G} * \mathrm{~T}$ & 847.029 & 1 & 847.029 & 3.802 & 0.051 \\
\hline $\mathrm{F}^{*} \mathrm{M}$ & 900.265 & 2 & 450.133 & 2.020 & 0.133 \\
\hline $\mathrm{T} * \mathrm{~F}$ & 797.844 & 2 & 398.922 & 1.790 & 0.167 \\
\hline $\mathrm{G} * \mathrm{~Hz}$ & 631.238 & 3 & 210.413 & 0.944 & 0.418 \\
\hline $\mathrm{G} * \mathrm{M}$ & 136.320 & 1 & 136.320 & 0.612 & 0.434 \\
\hline $\mathrm{D} * \mathrm{~F}$ & 299.528 & 2 & 149.764 & 0.672 & 0.511 \\
\hline$G * D$ & 84.737 & 1 & 84.737 & 0.380 & 0.538 \\
\hline$D * M$ & 43.561 & 1 & 43.561 & 0.196 & 0.658 \\
\hline$G * F$ & 136.588 & 2 & 68.294 & 0.307 & 0.736 \\
\hline $\mathrm{T} * \mathrm{~Hz}$ & 283.459 & 3 & 94.486 & 0.424 & 0.736 \\
\hline $\mathrm{D} * \mathrm{~T}$ & 18.559 & 1 & 18.559 & 0.083 & 0.773 \\
\hline $\mathrm{F}^{*} \mathrm{~Hz}$ & 300.583 & 6 & 50.097 & 0.225 & 0.969 \\
\hline $\mathrm{D} * \mathrm{~Hz}$ & 24.099 & 3 & 8.033 & 0.036 & 0.991 \\
\hline $\mathrm{G} * \mathrm{D} * \mathrm{~T}$ & 293.673 & 1 & 293.673 & 1.318 & 0.251 \\
\hline $\mathrm{T} * \mathrm{M} * \mathrm{~Hz}$ & 705.541 & 3 & 235.180 & 1.056 & 0.367 \\
\hline $\mathrm{G} * \mathrm{M} * \mathrm{~Hz}$ & 455.384 & 3 & 151.795 & 0.681 & 0.563 \\
\hline $\mathrm{G} * \mathrm{~T} * \mathrm{M}$ & 72.075 & 1 & 72.075 & 0.323 & 0.570 \\
\hline $\mathrm{T} * \mathrm{~F} * \mathrm{M}$ & 233.107 & 2 & 116.554 & 0.523 & 0.593 \\
\hline $\mathrm{D} * \mathrm{~T} * \mathrm{M}$ & 42.960 & 1 & 42.960 & 0.193 & 0.661 \\
\hline $\mathrm{G} * \mathrm{~T} * \mathrm{~Hz}$ & 314.386 & 3 & 104.795 & 0.470 & 0.703 \\
\hline$G * D * M$ & 18.213 & 1 & 18.213 & 0.082 & 0.775 \\
\hline $\mathrm{F}^{*} \mathrm{M} * \mathrm{~Hz}$ & 686.884 & 6 & 114.481 & 0.514 & 0.798 \\
\hline $\mathrm{D} * \mathrm{~T} * \mathrm{~F}$ & 96.868 & 2 & 48.434 & 0.217 & 0.805 \\
\hline $\mathrm{D} * \mathrm{~F} * \mathrm{~Hz}$ & 628.609 & 6 & 104.768 & 0.470 & 0.831 \\
\hline $\mathrm{D} * \mathrm{M} * \mathrm{~Hz}$ & 184.849 & 3 & 61.616 & 0.277 & 0.842 \\
\hline $\mathrm{G} * \mathrm{~F} * \mathrm{M}$ & 69.430 & 2 & 34.715 & 0.156 & 0.856 \\
\hline $\mathrm{D} * \mathrm{~F} * \mathrm{M}$ & 49.203 & 2 & 24.602 & 0.110 & 0.895 \\
\hline $\mathrm{G} * \mathrm{D} * \mathrm{~F}$ & 37.709 & 2 & 18.854 & 0.085 & 0.919 \\
\hline $\mathrm{T} * \mathrm{~F} * \mathrm{~Hz}$ & 434.057 & 6 & 72.343 & 0.325 & 0.924 \\
\hline $\mathrm{D} * \mathrm{~T} * \mathrm{~Hz}$ & 94.733 & 3 & 31.578 & 0.142 & 0.935 \\
\hline $\mathrm{G} * \mathrm{~T} * \mathrm{~F}$ & 19.393 & 2 & 9.696 & 0.044 & 0.957 \\
\hline $\mathrm{G} * \mathrm{D} * \mathrm{~Hz}$ & 39.713 & 3 & 13.238 & 0.059 & 0.981 \\
\hline $\mathrm{G} * \mathrm{~F} * \mathrm{~Hz}$ & 160.523 & 6 & 26.754 & 0.120 & 0.994 \\
\hline $\mathrm{G} * \mathrm{~T} * \mathrm{M} * \mathrm{~Hz}$ & 435.873 & 3 & 145.291 & 0.652 & 0.582 \\
\hline $\mathrm{D} * \mathrm{~T} * \mathrm{~F} * \mathrm{~Hz}$ & 1020.985 & 6 & 170.164 & 0.764 & 0.598 \\
\hline $\mathrm{G} * \mathrm{D} * \mathrm{~F} * \mathrm{M}$ & 195.121 & 2 & 97.561 & 0.438 & 0.645 \\
\hline $\mathrm{G} * \mathrm{D} * \mathrm{M} * \mathrm{~Hz}$ & 228.951 & 3 & 76.317 & 0.343 & 0.795 \\
\hline $\mathrm{D} * \mathrm{~T} * \mathrm{M} * \mathrm{~Hz}$ & 198.078 & 3 & 66.026 & 0.296 & 0.828 \\
\hline $\mathrm{G} * \mathrm{D} * \mathrm{~F} * \mathrm{~Hz}$ & 629.038 & 6 & 104.840 & 0.471 & 0.831 \\
\hline $\mathrm{G} * \mathrm{D} * \mathrm{~T} * \mathrm{M}$ & 8.401 & 1 & 8.401 & 0.038 & 0.846 \\
\hline $\mathrm{G} * \mathrm{D} * \mathrm{~T} * \mathrm{~Hz}$ & 140.564 & 3 & 46.855 & 0.210 & 0.889 \\
\hline $\mathrm{G} * \mathrm{D} * \mathrm{~T} * \mathrm{~F}$ & 47.557 & 2 & 23.778 & 0.107 & 0.899 \\
\hline $\mathrm{D} * \mathrm{~F} * \mathrm{M} * \mathrm{~Hz}$ & 449.599 & 6 & 74.933 & 0.336 & 0.918 \\
\hline $\mathrm{G} * \mathrm{~T} * \mathrm{~F} * \mathrm{~Hz}$ & 397.663 & 6 & 66.277 & 0.297 & 0.938 \\
\hline $\mathrm{D} * \mathrm{~T} * \mathrm{~F} * \mathrm{M}$ & 26.480 & 2 & 13.240 & 0.059 & 0.942 \\
\hline $\mathrm{G} * \mathrm{~T} * \mathrm{~F} * \mathrm{M}$ & 25.455 & 2 & 12.727 & 0.057 & 0.944 \\
\hline $\mathrm{G} * \mathrm{~F} * \mathrm{M} * \mathrm{~Hz}$ & 310.177 & 6 & 51.696 & 0.232 & 0.966 \\
\hline $\mathrm{T} * \mathrm{~F} * \mathrm{M} * \mathrm{~Hz}$ & 124.919 & 6 & 20.820 & 0.093 & 0.997 \\
\hline $\mathrm{G} * \mathrm{D} * \mathrm{~T} * \mathrm{~F} * \mathrm{~Hz}$ & 985.768 & 6 & 164.295 & 0.737 & 0.620 \\
\hline $\mathrm{G} * \mathrm{D} * \mathrm{~T} * \mathrm{M} * \mathrm{~Hz}$ & 250.699 & 3 & 83.566 & 0.375 & 0.771 \\
\hline $\mathrm{D} * \mathrm{~T} * \mathrm{~F} * \mathrm{M} * \mathrm{~Hz}$ & 478.625 & 6 & 79.771 & 0.358 & 0.905 \\
\hline $\mathrm{G} * \mathrm{D} * \mathrm{~T} * \mathrm{~F} * \mathrm{M}$ & 14.766 & 2 & 7.383 & 0.033 & 0.967 \\
\hline $\mathrm{G} * \mathrm{D} * \mathrm{~F} * \mathrm{M} * \mathrm{~Hz}$ & 243.310 & 6 & 40.552 & 0.182 & 0.982 \\
\hline $\mathrm{G} * \mathrm{~T} * \mathrm{~F} * \mathrm{M} * \mathrm{~Hz}$ & 234.439 & 6 & 39.073 & 0.175 & 0.984 \\
\hline Error & 434484.499 & 1950 & 222.813 & & \\
\hline Total & 575097.950 & 2160 & & & \\
\hline Corrected Total & 481022.869 & 2159 & & & \\
\hline
\end{tabular}




\subsubsection{Statistical power for Experiment 2}

As in Experiment 1, Equations 3-2 and 3-3 were used to evaluate the statistical power of Experiment 2 for main effects and two-way interactions, respectively. Also like Experiment 1, the "worst case" or lowest statistical power is that associated with vibration frequency (5 levels). The variance of the response variable (force-recall error) for Experiment 2 was found to be $223 \mathrm{~N}^{2}$. The results of applying Equation 3-2 to the data collected in Experiment 2 are contained in Table 4.2.4. As indicated in the table, with this experiment's observed variance and sample size, the statistical test for evaluating this main effect demonstrated a sensitivity of $5.2 \mathrm{~N}$ with a power of $0.90,4.6 \mathrm{~N}$ with a power of 0.80 , and $4.0 \mathrm{~N}$ with a power of 0.70 .

Table 4.2.4 Sensitivity of Experiment 2 for detecting force-recall error differences due to vibration frequency effects at three levels of type II error probability $(\beta)$.

\begin{tabular}{|c|c|c|c|c|c|c|c|c|c|c|}
\hline $\begin{array}{l}\text { Sensitivity } \\
\text { (Newtons) }\end{array}$ & $\begin{array}{c}\text { Parameter } \\
\text { value } \\
\text { (from table) }\end{array}$ & $\begin{array}{l}\text { Levels of } \\
\text { factor a }\end{array}$ & $\begin{array}{l}\text { Observed } \\
\text { variance }\end{array}$ & $\begin{array}{c}\text { Number } \\
\text { of } \\
\text { subjects }\end{array}$ & $\begin{array}{c}\text { Trials at } \\
\text { each } \\
\text { level }\end{array}$ & $\begin{array}{c}\text { Replicates } \\
\text { at each } \\
\text { level }\end{array}$ & $\begin{array}{c}\text { Numerator } \\
\text { DF } \\
(\mathrm{a}-1)\end{array}$ & $\begin{array}{c}\text { Denominator } \\
\text { DF } \\
a(n-1)\end{array}$ & $\begin{array}{l}\text { Type II } \\
\text { error }\end{array}$ & $\begin{array}{l}\text { Type I } \\
\text { error }\end{array}$ \\
\hline D & $\Phi$ & $\mathbf{a}$ & $\sigma^{2}$ & & & n & $v_{1}$ & $v_{2}$ & $1-\beta$ & 1- $\alpha$ \\
\hline 5.2 & 1.7 & 5 & 223 & 20 & 12 & 240 & 4 & 1195 & 0.90 & 0.95 \\
\hline 4.6 & 1.5 & 5 & 223 & 20 & 12 & 240 & 4 & 1195 & 0.80 & 0.95 \\
\hline 4.0 & 1.3 & 5 & 223 & 20 & 12 & 240 & 4 & 1195 & 0.70 & 0.95 \\
\hline
\end{tabular}

As in Experiment 1, the worst case for testing two-way interactions in Experiment 2 is represented by the vibration frequency ( 5 levels) by force level (3 levels) interaction. The results of applying Equation 3-3 to the data collected in Experiment 2 are contained in 
Table 4.2.5. As indicated in this table, with this experiment's observed variance and sample size, the statistical test for evaluating this interaction effect demonstrated a sensitivity of $10.6 \mathrm{~N}$ with a power of $0.90,9.2 \mathrm{~N}$ with a power of 0.80 , and $8.5 \mathrm{~N}$ with a power of 0.70 .

Table 4.2.5 Sensitivity of Experiment 2 for detecting force-recall error differences due to the vibration frequency by force level interaction effects at three levels of type II error probability ( $\beta$ ).

\begin{tabular}{|c|c|c|c|c|c|c|c|c|c|c|c|}
\hline $\begin{array}{l}\text { Sensitivity } \\
\text { (Newtons) }\end{array}$ & $\begin{array}{c}\text { Parameter } \\
\text { value } \\
\text { (from table) }\end{array}$ & $\begin{array}{c}\text { Levels } \\
\text { of factor } \\
\text { a }\end{array}$ & $\begin{array}{c}\text { Levels } \\
\text { of factor } \\
b\end{array}$ & $\begin{array}{l}\text { Observed } \\
\text { variance }\end{array}$ & $\begin{array}{c}\text { Number } \\
\text { of } \\
\text { subjects }\end{array}$ & $\begin{array}{c}\text { Trials } \\
\text { at each } \\
\text { level }\end{array}$ & $\begin{array}{l}\text { Replicates } \\
\text { at each } \\
\text { level }\end{array}$ & $\begin{array}{c}\text { Numerator } \\
\text { DF } \\
(a-1)(b-1)\end{array}$ & $\begin{array}{c}\text { Denominator } \\
\text { DF } \\
a b(n-1)\end{array}$ & $\begin{array}{l}\text { Type II } \\
\text { error }\end{array}$ & $\begin{array}{l}\text { Type I } \\
\text { error }\end{array}$ \\
\hline D & $\Phi$ & $\mathbf{a}$ & b & $\sigma^{2}$ & & & n & $v_{1}$ & $v_{2}$ & $1-\beta$ & 1- $\alpha$ \\
\hline 10.6 & 1.5 & 5 & 3 & 223 & 20 & 4 & 80 & 8 & 1185 & 0.90 & 0.95 \\
\hline 9.2 & 1.3 & 5 & 3 & 223 & 20 & 4 & 80 & 8 & 1185 & 0.80 & 0.95 \\
\hline 8.5 & 1.2 & 5 & 3 & 223 & 20 & 4 & 80 & 8 & 1185 & 0.70 & 0.95 \\
\hline
\end{tabular}

\subsubsection{Vibration frequency effects in Experiment 2}

Table 4.2.6 contains the means, standard deviations, and 95\% confidence intervals for recall errors for each frequency of vibration exposure employed in Experiment 2.

Table 4.2.6 Force-recall error means (in Newtons) at each vibration frequency in Experiment 2.

\begin{tabular}{ccccc}
\hline $\begin{array}{c}\text { Vibration } \\
\text { Freq in Hz }\end{array}$ & \multirow{2}{*}{ Mean } & SD & \multicolumn{2}{c}{$95 \%$ Confidence Interval } \\
\cline { 4 - 5 } & & & Lower Bound & Upper Bound \\
\hline 0 & 3.3 & 13.5 & 1.6 & 5.1 \\
16 & 4.8 & 12.8 & 3.6 & 5.9 \\
31.5 & 8.0 & 15.9 & 6.5 & 9.4 \\
63 & 8.8 & 16.0 & 7.3 & 10.2 \\
125 & 6.5 & 15.0 & 5.2 & 7.9 \\
\hline
\end{tabular}


As depicted in Figures 4.2.2 through 4.2.5, vibration frequency effects were curvilinear in nature with greatest errors produced at 31.5 and $63 \mathrm{~Hz}$. As also shown, vibration frequency affected grip and push errors similarly.

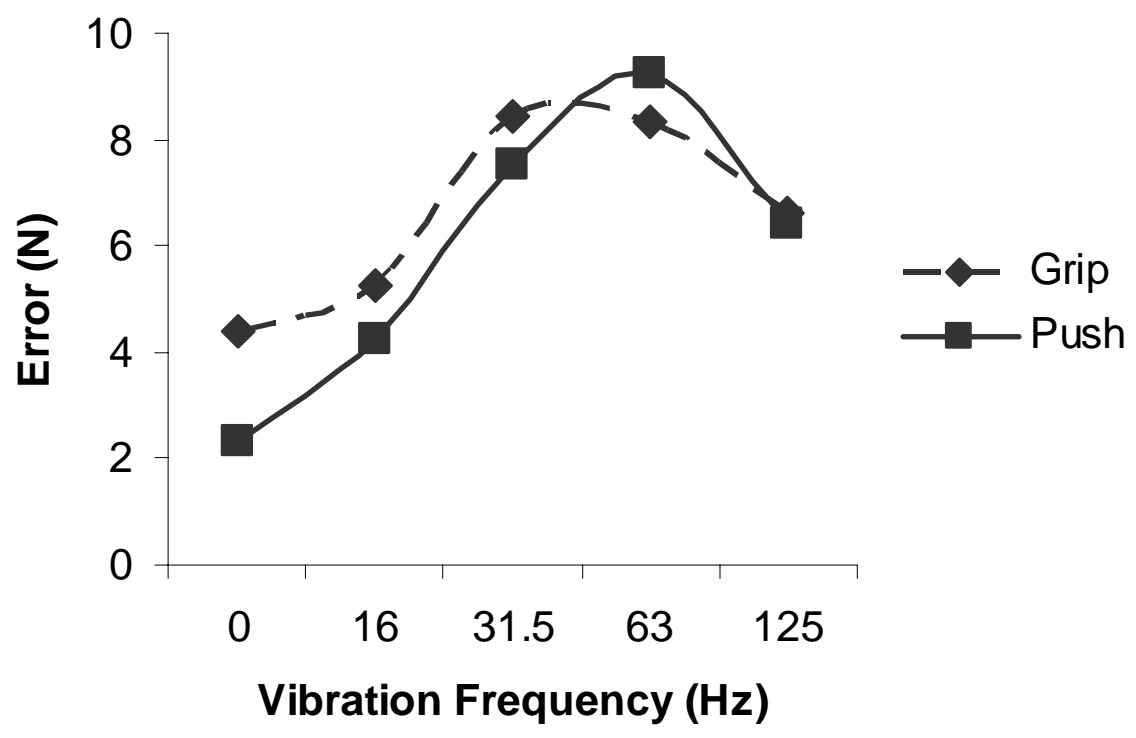

Figure 4.2.2 Experiment 2 grip and push force-recall error means plotted against vibration frequency across all force levels. 


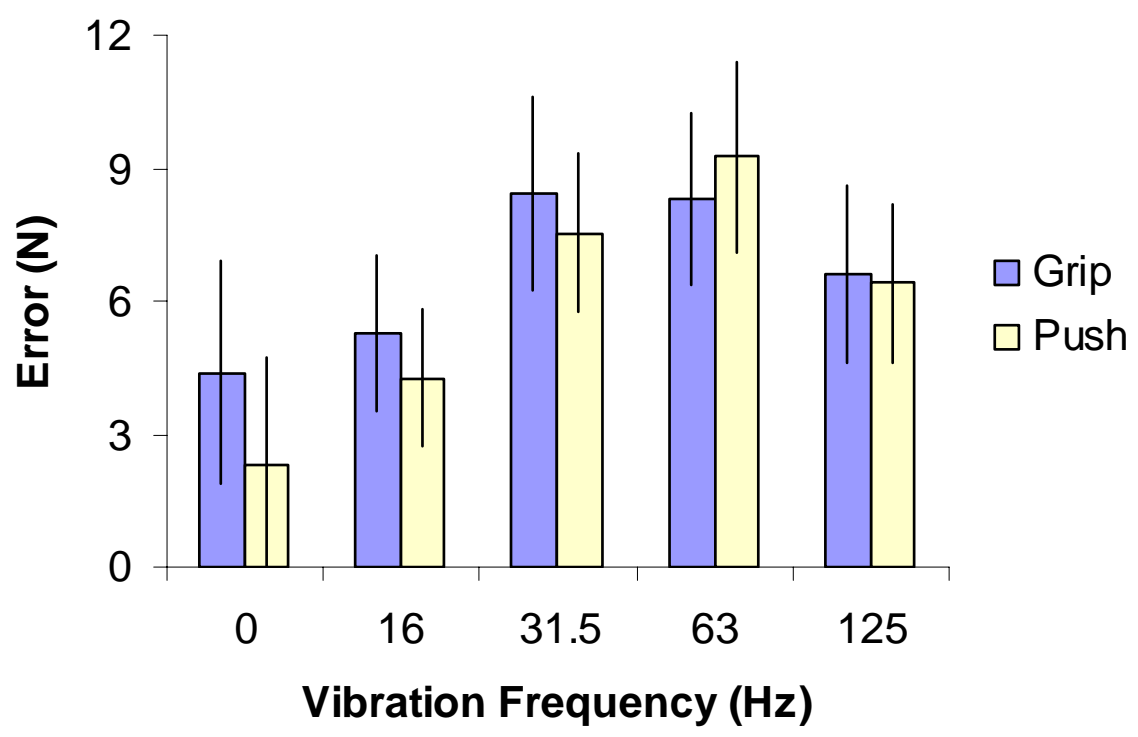

Figure 4.2.3 Experiment 2 grip and push force-recall error means plotted against vibration frequency. (Y error bars $=95 \%$ C.I.)

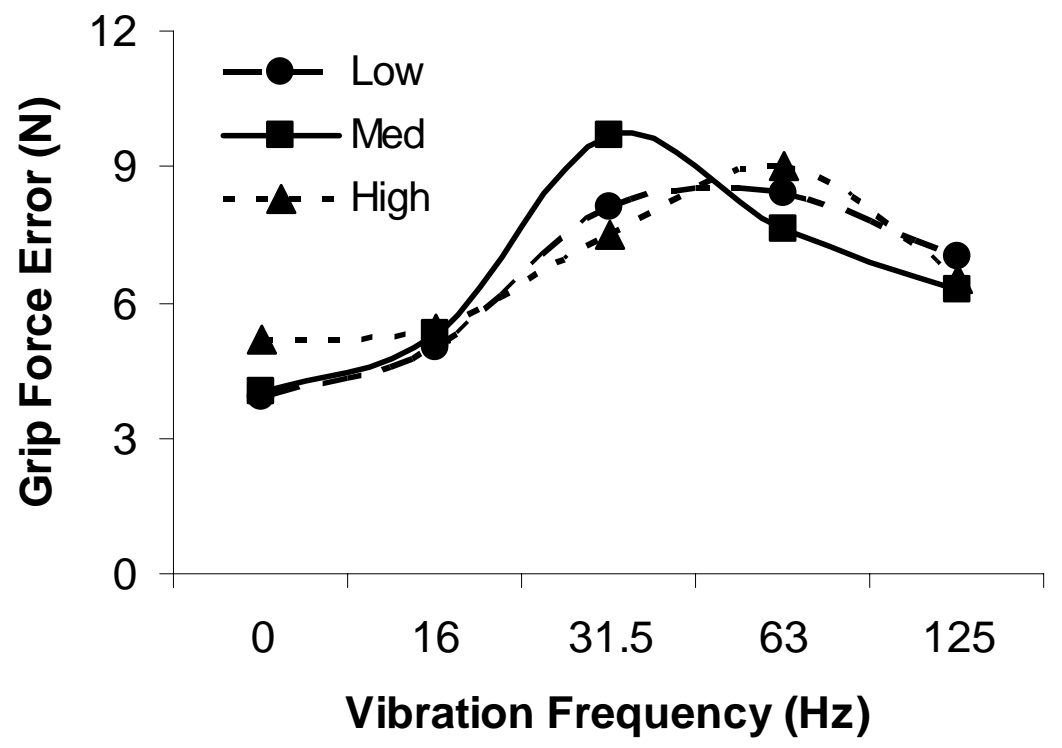

Figure 4.2.4 Experiment 2 grip force-recall error means for low, medium, and high target force levels plotted against vibration frequency. 


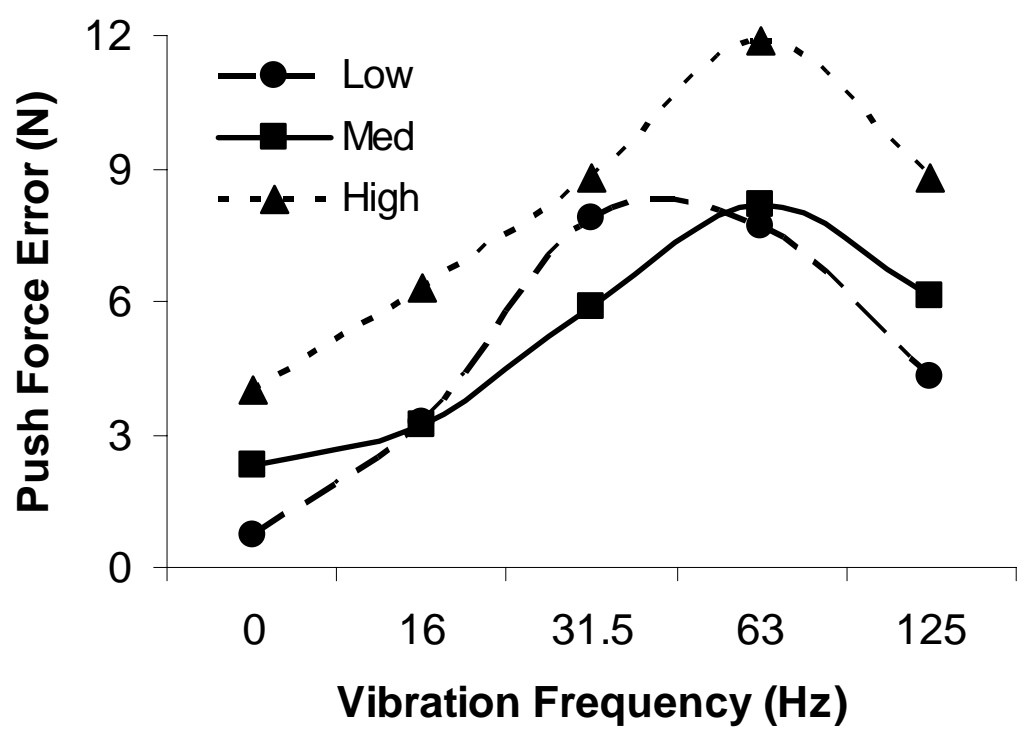

Figure 4.2.5 Experiment 2 push force-recall error means for low, medium, and high target force levels plotted against vibration frequency.

Pairwise comparisons using Tukey's HSD procedure are contained in Table 4.2.7. The results showed that the means of force-recall errors at $31.5 \mathrm{~Hz}$ and $63 \mathrm{~Hz}$ were each significantly different than the means at $0 \mathrm{~Hz}$ and $16 \mathrm{~Hz}$, but were not statistically different from each other or from the error mean at $125 \mathrm{~Hz}$. Error means for $125 \mathrm{~Hz}$ and $16 \mathrm{~Hz}$ were statistically equivalent, but the mean for $125 \mathrm{~Hz}$ was suggestively different from the zero vibration condition. 
Table 4.2.7 Tukey's HSD pair-wise comparisons for vibration frequency in Experiment 2.

\begin{tabular}{|c|c|c|c|c|c|c|}
\hline \multirow{2}{*}{$\begin{array}{c}\text { (I) } \\
\text { Freq. } \\
(\mathrm{Hz}) .\end{array}$} & \multirow{2}{*}{$\begin{array}{c}(\mathrm{J}) \\
\text { Freq. } \\
(\mathrm{Hz}) .\end{array}$} & \multirow{2}{*}{$\begin{array}{c}\text { Mean } \\
\text { Difference } \\
(\mathrm{I}-\mathrm{J})\end{array}$} & \multirow[b]{2}{*}{ Std. Error } & \multirow[b]{2}{*}{ Sig. } & \multicolumn{2}{|c|}{$\begin{array}{l}\text { 95\% Confidence Interval for } \\
\text { Difference } \\
\end{array}$} \\
\hline & & & & & Lower Bound & Upper Bound \\
\hline \multirow[t]{4}{*}{0.0} & 16.0 & -1.4 & 1.18 & 0.752 & -4.6 & 1.8 \\
\hline & 31.5 & -4.6 & 1.18 & 0.001 & -7.9 & -1.4 \\
\hline & 63.0 & -5.4 & 1.18 & 0.001 & -8.7 & -2.2 \\
\hline & 125.0 & -3.2 & 1.18 & 0.056 & -6.4 & 0.1 \\
\hline \multirow[t]{4}{*}{16.0} & 0.0 & 1.4 & 1.18 & 0.752 & -1.8 & 4.6 \\
\hline & 31.5 & -3.2 & 0.96 & 0.008 & -5.8 & -0.6 \\
\hline & 63.0 & -4.0 & 0.96 & 0.001 & -6.7 & -1.4 \\
\hline & 125.0 & -1.8 & 0.96 & 0.361 & -4.4 & 0.9 \\
\hline \multirow[t]{4}{*}{31.5} & 0.0 & 4.6 & 1.18 & 0.001 & 1.4 & 7.9 \\
\hline & 16.0 & 3.2 & 0.96 & 0.008 & 0.6 & 5.8 \\
\hline & 63.0 & -0.8 & 0.96 & 0.919 & -3.4 & 1.8 \\
\hline & 125.0 & 1.5 & 0.96 & 0.553 & -1.2 & 4.1 \\
\hline \multirow[t]{4}{*}{63.0} & 0.0 & 5.4 & 1.18 & 0.001 & 2.2 & 8.7 \\
\hline & 16.0 & 4.0 & 0.96 & 0.001 & 1.4 & 6.7 \\
\hline & 31.5 & 0.8 & 0.96 & 0.919 & -1.8 & 3.4 \\
\hline & 125.0 & 2.3 & 0.96 & 0.129 & -0.4 & 4.9 \\
\hline \multirow[t]{4}{*}{125.0} & 0.0 & 3.2 & 1.18 & 0.056 & -0.1 & 6.4 \\
\hline & 16.0 & 1.8 & 0.96 & 0.361 & -0.9 & 4.4 \\
\hline & 31.5 & -1.5 & 0.96 & 0.553 & -4.1 & 1.2 \\
\hline & 63.0 & -2.3 & 0.96 & 0.129 & -4.9 & 0.4 \\
\hline
\end{tabular}

\subsubsection{Exertion type effects in Experiment 2}

As in Experiment 1, the ANOVA for Experiment 2 revealed no differences between the error means for recalled grip error and recalled push error. The means for error for each exertion type are contained in Table 4.2.8. 
Table 4.2.8 Grip and push force-recall error means (in Newtons) in Experiment 2.

\begin{tabular}{ccccc}
\hline $\begin{array}{c}\text { Exertion } \\
\text { Type }\end{array}$ & Mean & SD & \multicolumn{2}{c}{ 95\% Confidence Interval } \\
\cline { 4 - 5 } & & & Lower Bound & Upper Bound \\
\hline Grip & 6.8 & 15.5 & 5.9 & 7.8 \\
Push & 6.4 & 14.4 & 5.5 & 7.2 \\
\hline
\end{tabular}

\subsubsection{Vibration magnitude effects in Experiment 2}

Experiment 2 explored two levels of vibration magnitude for the four frequencies of single-axis sinusoidal vibration. The recalled force error means for these eight vibration exposure conditions were analyzed along with the zero vibration condition. The means, standard deviations, and 95\% confidence intervals are contained in Table 4.2.9. The ANOVA revealed that vibration magnitude was a significant main effect. Tukey's HSD pairwise comparisons indicated that the higher magnitude of vibration exposure produced significantly greater recalled force errors than either the lower level of vibration magnitude or the zero vibration condition. The error means for low vibration magnitude and zero vibration were statistically equivalent.

Table 4.2.9 Means (in Newtons) for force-recall error at the zero vibration condition and at each level of vibration magnitude used in Experiment 2.

\begin{tabular}{ccccc}
\hline $\begin{array}{c}\text { Vibration } \\
\text { Magnitude }\end{array}$ & \multirow{2}{*}{ Mean } & \multirow{2}{*}{ SD } & \multicolumn{2}{c}{$95 \%$ Confidence Interval } \\
\cline { 4 - 5 } & & & Lower Bound & Upper Bound \\
\hline Zero & 3.3 & 13.5 & 1.6 & 5.1 \\
Low & 4.9 & 13.4 & 4.0 & 5.7 \\
High & 9.1 & 16.2 & 8.1 & 10.2 \\
\hline
\end{tabular}


The ANOVA for Experiment 2 revealed a significant interaction between vibration frequency and vibration magnitude. This interaction is demonstrated in Table 4.2.10, Figure 4.2.6, and Figure 4.2.7. As indicated, the effect of increased vibration magnitude was significantly more pronounced at $31.5 \mathrm{~Hz}$ and $63 \mathrm{~Hz}$.

Table 4.2.10 Means (in Newtons) of force-recall error at each level of vibration frequency and magnitude combination used in Experiment 2.

\begin{tabular}{cccccc}
\hline $\begin{array}{c}\text { Vibration } \\
\text { Freq in Hz }\end{array}$ & $\begin{array}{c}\text { Vibration } \\
\text { Magnitude }\end{array}$ & Mean & SD & \multicolumn{2}{c}{ 95\% Confidence Interval } \\
& Low & 3.9 & 11.4 & 2.0 & Lower Bound Upper Bound \\
\hline \multirow{2}{*}{16} & High & 5.6 & 14.0 & 3.7 & 7.5 \\
& Low & 4.8 & 14.4 & 2.9 & 6.7 \\
\multirow{2}{*}{63.5} & High & 11.2 & 16.7 & 9.3 & 13.1 \\
& Low & 5.2 & 13.9 & 3.3 & 7.1 \\
& High & 12.4 & 17.2 & 10.5 & 14.2 \\
& Low & 5.6 & 13.9 & 3.7 & 7.5 \\
& High & 7.5 & 16.1 & 5.6 & 9.3 \\
\hline
\end{tabular}




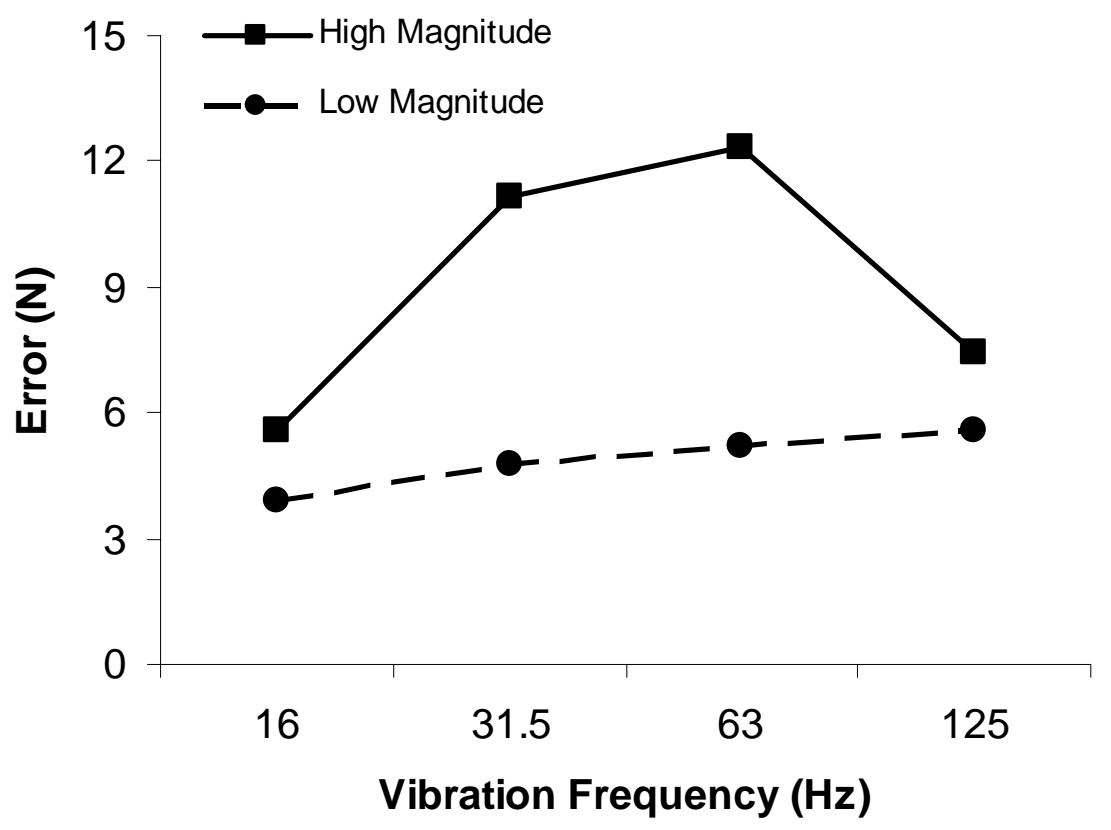

Figure 4.2.6 Force-recall error as a function of vibration frequency and vibration magnitude across all conditions of Experiment 2.

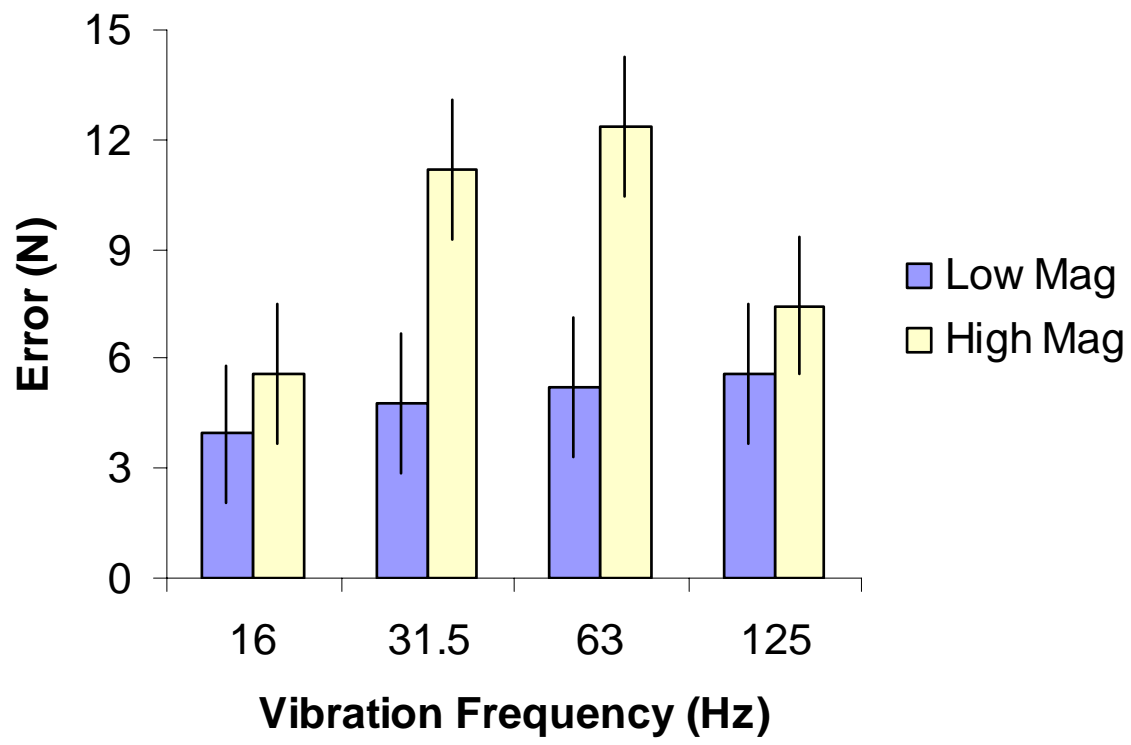

Figure 4.2.7 Force-recall error as a function of vibration frequency and vibration magnitude in Experiment 2. (Y error bars $=95 \%$ C.I.) 
The ANOVA for Experiment 2 also identified a significant interaction between exertion type (grip or push) and vibration magnitude. Table 4.2.11 contains the means for force-recall error for grip and for push at each level of vibration magnitude. Figure 4.2.8 and Figure 4.2.9 illustrate the effect of this interaction.

Table 4.2.11 Means (in Newtons) for grip and push force-recall error at each level of vibration magnitude exposure in Experiment 2.

\begin{tabular}{cccccc}
\hline $\begin{array}{c}\text { Exertion } \\
\text { Type }\end{array}$ & $\begin{array}{c}\text { Vibration } \\
\text { Magnitude }\end{array}$ & Mean & SD & \multicolumn{2}{c}{ 95\% Confidence Interval } \\
\hline \multirow{3}{*}{ Grip } & Zero & 4.4 & 13.9 & 1.7 & Lower Bound \\
& Low & 5.9 & 14.3 & 4.5 & 7.2 \\
& High & 8.4 & 16.7 & 7.1 & 9.8 \\
& Zero & 2.3 & 13.1 & -0.4 & 5.0 \\
\multirow{3}{*}{ Push } & Low & 3.9 & 12.4 & 2.6 & 5.2 \\
& High & 9.8 & 15.7 & 8.5 & 11.2 \\
\hline
\end{tabular}




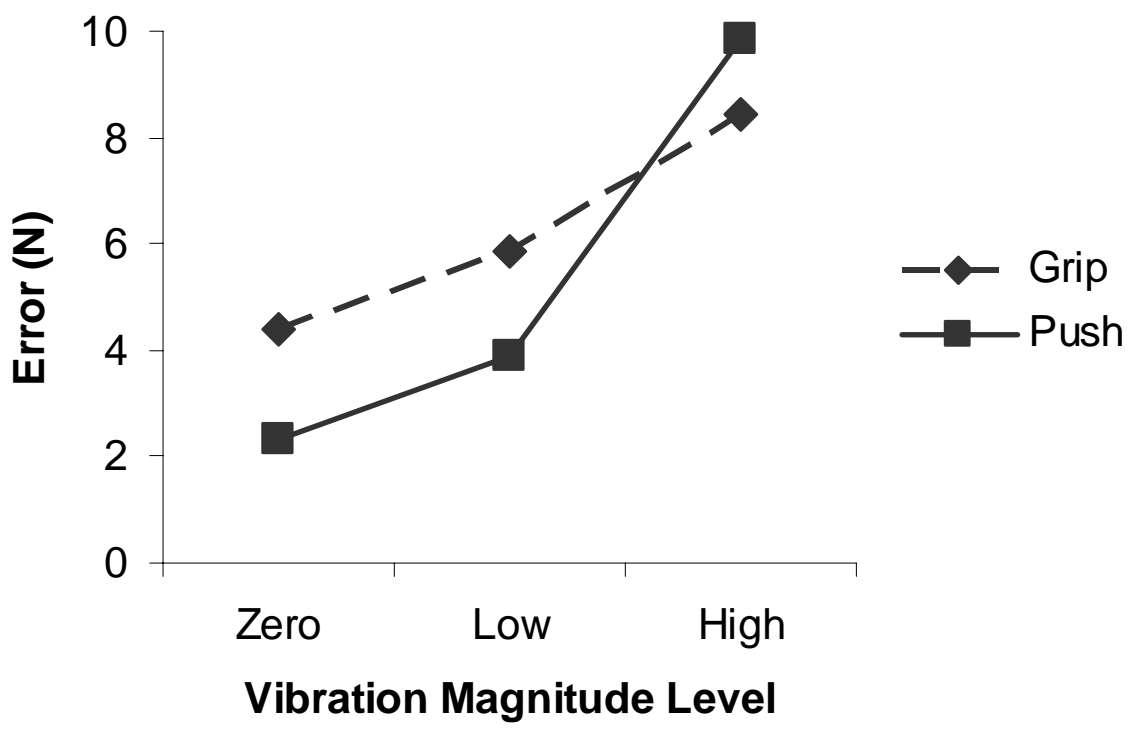

Figure 4.2.8 Force-recall error as a function of vibration magnitude and exertion type (grip or push) across all conditions of Experiment 2.

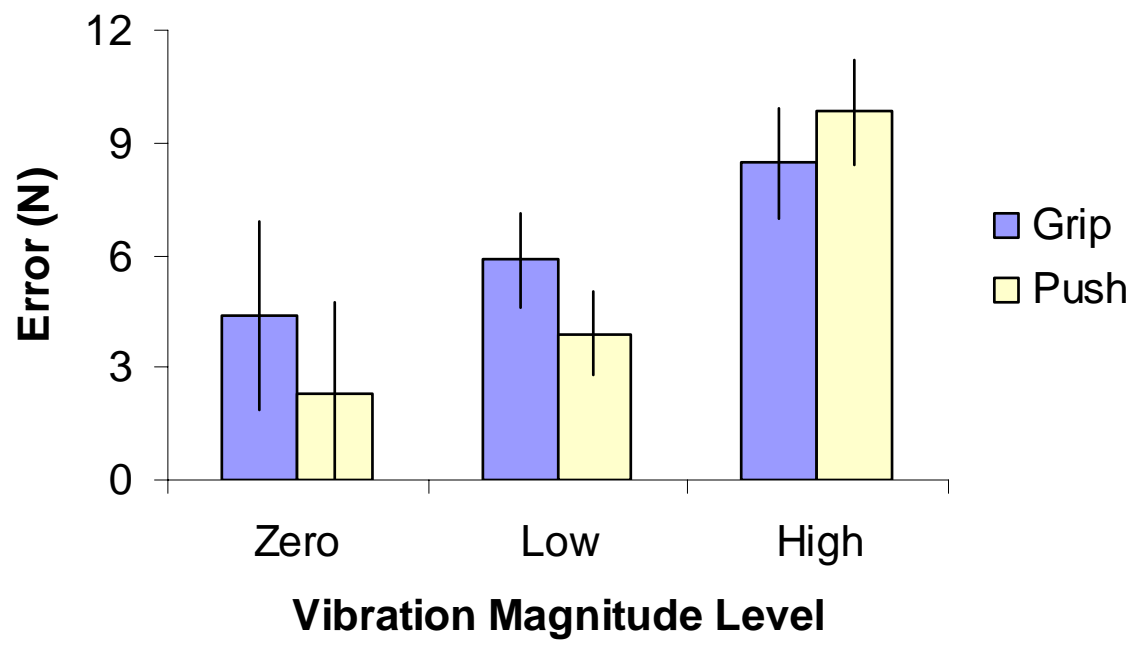

Figure 4.2.9 Force-recall error as a function of vibration magnitude and exertion type (grip or push) in Experiment 2. (Y error bars $=95 \%$ C.I.) 


\subsubsection{Force level effects in Experiment 2}

The relationship between force level and force-recall error means in Experiment 2 is demonstrated in Table 4.2.12, Figure 4.2.10, and Figure 4.2.11. Across exertion type (grip or push), force level was found to be significant. As shown in the figure, accuracy of grip recall was virtually unchanged by force level. However, push force-recall error was higher at the high force level than at the other two force levels, although the interaction between force level and exertion type was not found to be statistically significant.

Table 4.2.12 Means (in Newtons) for force-recall error at each level of applied force in Experiment 2.

\begin{tabular}{ccccc}
\hline \multirow{2}{*}{ Force Level } & \multirow{2}{*}{ Mean } & \multirow{2}{*}{ SD } & \multicolumn{2}{c}{ 95\% Confidence Interval } \\
\cline { 4 - 5 } & & 10.0 & 5.3 & 6.7 \\
\hline Low & 6.0 & 14.7 & 5.1 & 7.2 \\
Med & 6.2 & 18.7 & 6.3 & 9.0 \\
High & 7.6 & & & \\
\hline
\end{tabular}




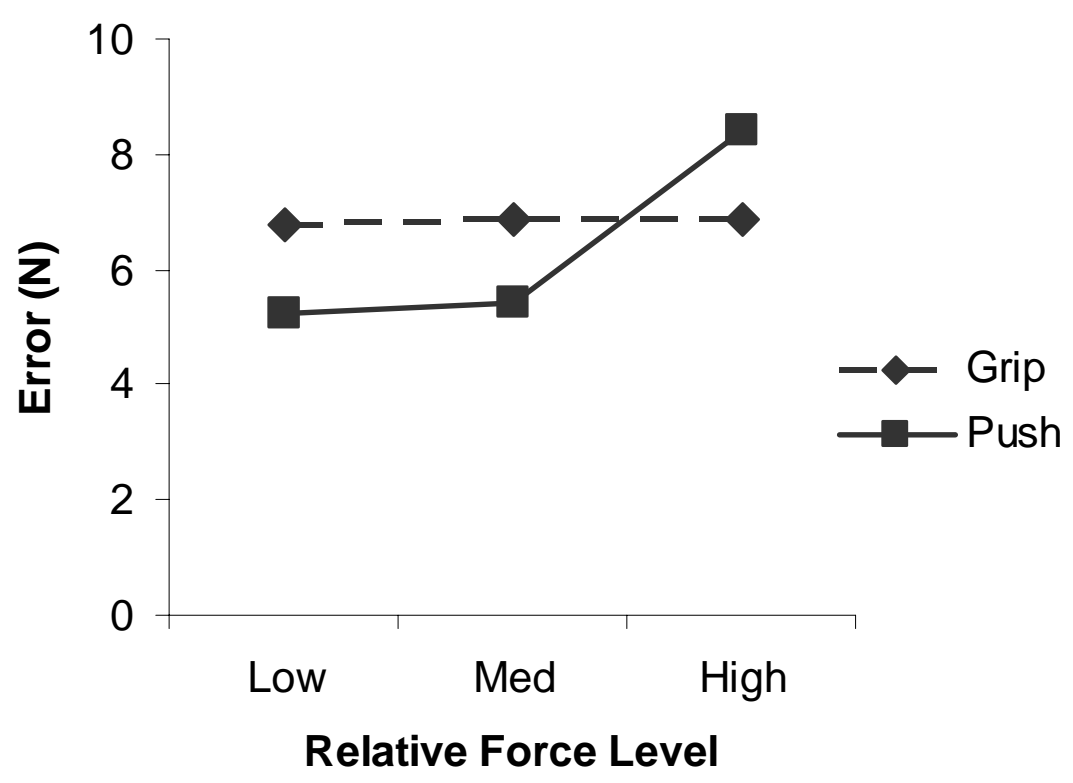

Figure 4.2.10 Force-recall error for grip and push plotted against relative force level for Experiment 2.

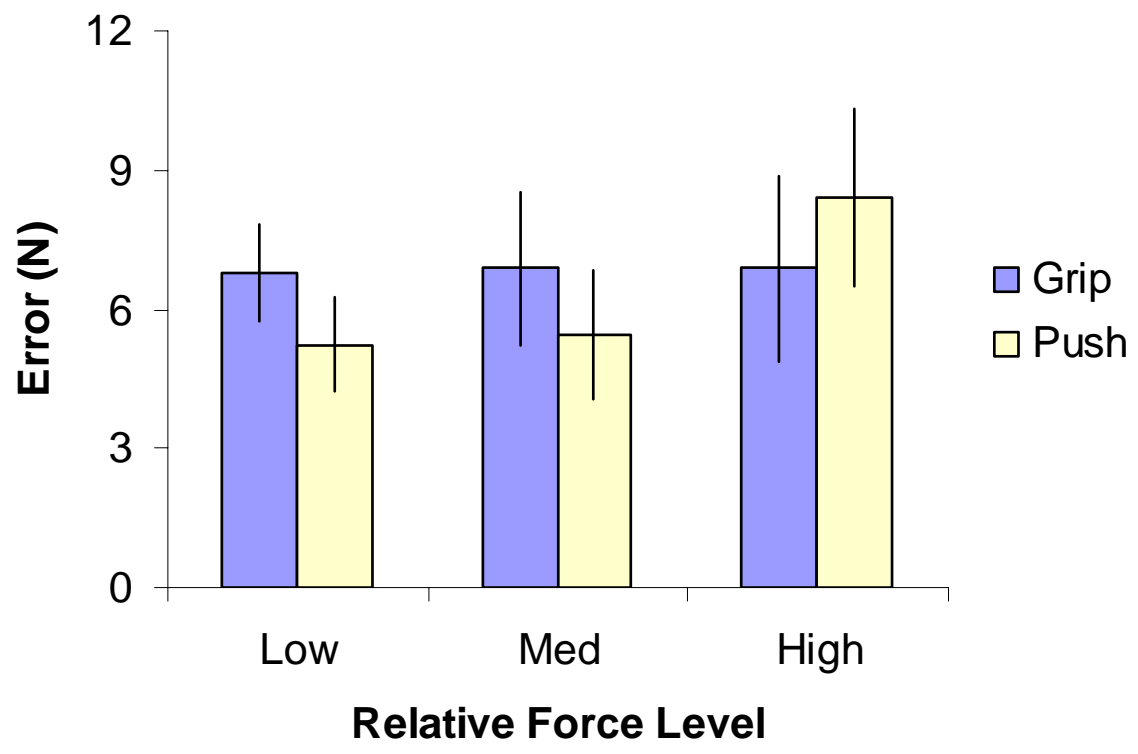

Figure 4.2.11 Force-recall error for grip and push plotted against relative force level for Experiment 2. ( $\mathrm{Y}$ error bars $=95 \%$ C.I.) 


\subsubsection{Test-retest reliability in Experiment 2}

The ANOVA revealed a significant difference in recall error means between test days in Experiment 2. As indicated in Table 4.2.13, subjects were more accurate on the second day of testing than first day.

To further examine test-retest reliability, the actual grip and push recall forces produced in Day 1 were compared with those of Day 2. As displayed in Table 4.2.14, the Pearson productmoment correlation results indicated strong test-retest reliability as correlations for all but one subject were found to be significant for both grip and push recall forces. The lone exception (male subject M8) displayed significant test-retest reliability for push force, but not for grip force.

Table 4.2.13 Means (in Newtons) for force-recall error for each test session in Experiment 2.

\begin{tabular}{ccccc}
\hline \multirow{2}{*}{ Day } & \multirow{2}{*}{ Mean } & \multirow{2}{*}{ SD } & \multicolumn{2}{c}{ 95\% Confidence Interval } \\
\cline { 4 - 5 } & & & Lower Bound & Upper Bound \\
\hline Day 1 & 7.4 & 15.9 & 6.4 & 8.3 \\
Day 2 & 5.8 & 13.8 & 5.0 & 6.6 \\
\hline
\end{tabular}


Table 4.2.14 Test-retest correlations for recalled grip and push forces for each subject across all conditions for Experiment 2.

\begin{tabular}{cll}
\hline Subject & Grip & Push \\
\hline F1 & $0.77^{*}$ & $0.93^{*}$ \\
F2 & $0.73^{*}$ & $0.81^{*}$ \\
F3 & $0.76^{*}$ & $0.78^{*}$ \\
F4 & $0.88^{*}$ & $0.94^{*}$ \\
F5 & $0.65^{*}$ & $0.83^{*}$ \\
F6 & $0.58^{*}$ & $0.83^{*}$ \\
F7 & $0.69^{*}$ & $0.92^{*}$ \\
F8 & $0.71^{*}$ & $0.91 *$ \\
F9 & $0.69^{*}$ & $0.94^{*}$ \\
F10 & $0.83^{*}$ & $0.91 *$ \\
M1 & $0.54^{*}$ & $0.86^{*}$ \\
M2 & $0.64^{*}$ & $0.90^{*}$ \\
M3 & $0.64^{*}$ & $0.92^{*}$ \\
M4 & $0.91^{*}$ & $0.92^{*}$ \\
M5 & $0.74^{*}$ & $0.85^{*}$ \\
M6 & $0.95^{*}$ & $0.93^{*}$ \\
M7 & $0.51^{*}$ & $0.63^{*}$ \\
M8 & 0.30 & $0.86^{*}$ \\
M9 & $0.45^{*}$ & $0.61 *$ \\
M10 & $0.79^{*}$ & $0.90^{*}$ \\
\hline Mean & $0.69^{*}$ & $0.86^{*}$ \\
Min & 0.30 & $0.61 *$ \\
Max & $0.95^{*}$ & $0.94^{*}$ \\
\hline significant at 0.01 level $(2-t a i 1 e d)$
\end{tabular}

*significant at 0.01 level (2-tailed) 


\subsubsection{Gender effects in Experiment 2}

The ANOVA for Experiment 2 revealed a significant gender effect. As the data in Table 4.2.15 indicate, females were more accurate than males in recalling the applied forces.

Table 4.2.15 Means (in Newtons) for force-recall error for each gender in Experiment 2.

\begin{tabular}{ccccc}
\hline \multirow{2}{*}{ Gender } & \multirow{2}{*}{ Mean } & \multirow{2}{*}{ SD } & \multicolumn{2}{c}{$95 \%$ Confidence Interval } \\
\cline { 4 - 5 } & & & Lower Bound & Upper Bound \\
\hline Female & 4.9 & 16.2 & 3.9 & 5.9 \\
Male & 8.3 & 13.3 & 7.5 & 9.1 \\
\hline
\end{tabular}

An examination of force recall error means for the 20 individual subjects revealed that for grip force recall, the six most accurate subjects (and 7 of the top 10) were female. For push force recall, 8 of the 12 most accurate subjects were female.

The gender by exertion type interaction was found to be suggestively significant in Experiment 2. The means for this interaction are contained in Table 4.2.16. As shown in Figure 4.2.12 and Figure 4.2.13, for males, force-recall errors for grip and push were about the same. However, females tended to be more accurate at recalling push forces than grip forces. 
Table 4.2.16 Means (in Newtons) for force-recall error for each gender and exertion type (grip or push) across all conditions of Experiment 2.

\begin{tabular}{cccccc}
\hline \multirow{2}{*}{ Gender } & $\begin{array}{c}\text { Exertion } \\
\text { Type }\end{array}$ & Mean & & SD & \multicolumn{2}{c}{ 95\% Confidence Interval } \\
\cline { 5 - 6 } Female & Grip & 5.9 & 18.2 & 4.6 & Lower Bound \\
& Push & 3.9 & 13.9 & 2.7 & 5.2 \\
\multirow{2}{*}{ Male } & Grip & 7.8 & 12.0 & 6.5 & 9.1 \\
& Push & 8.8 & 14.5 & 7.5 & 10.0 \\
\hline
\end{tabular}

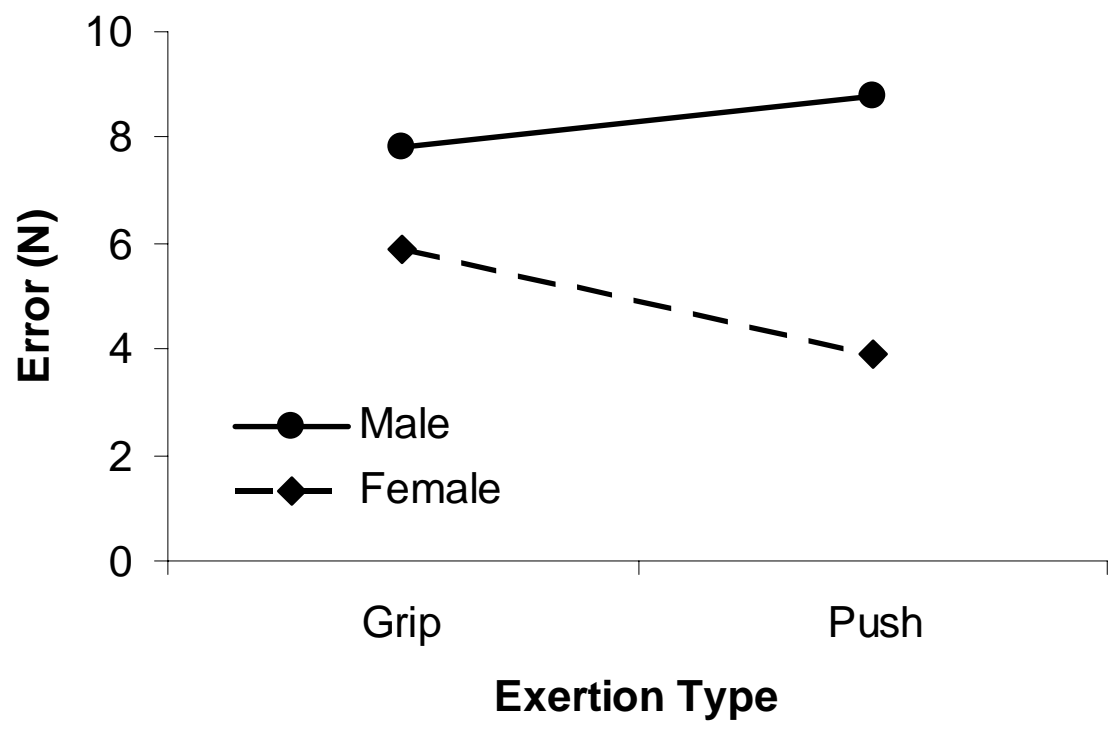

Figure 4.2.12 Interaction between gender and exertion type (grip or push) across all conditions of Experiment 2. 


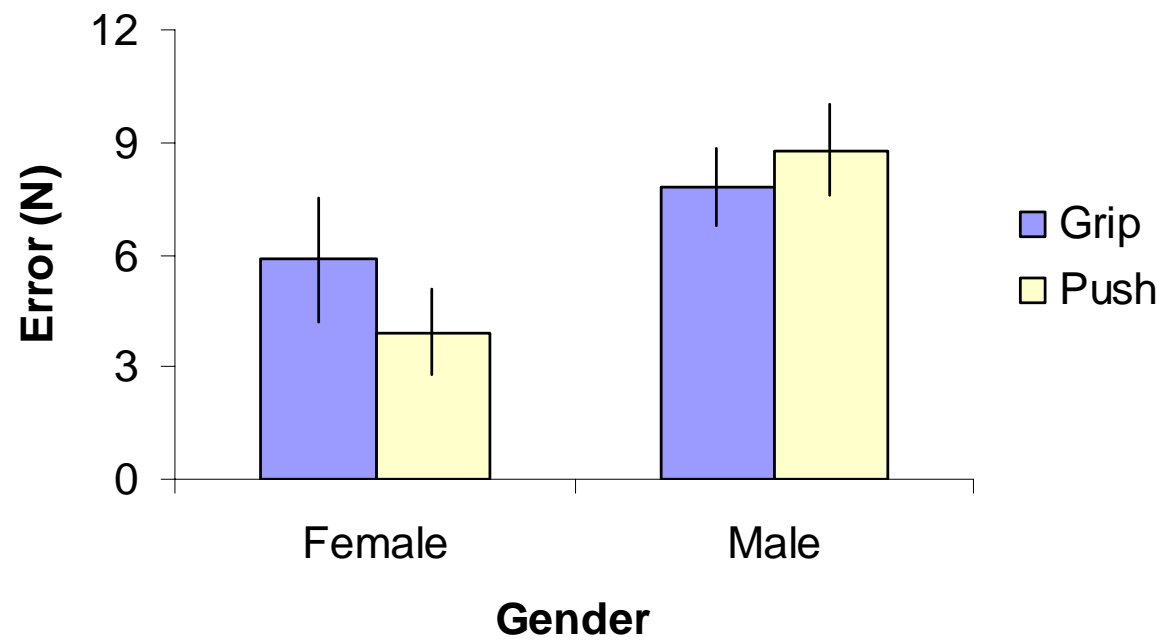

Figure 4.2.13 Interaction between gender and exertion type (grip or push) in Experiment 2. ( $\mathrm{Y}$ error bars $=95 \%$ C.I.)

\subsection{Some combined results of the two experiments}

\subsubsection{Applied grip and push forces for Experiments $1 \& 2$}

While most of the results are reported in terms of force-recall error, presented below are data regarding the magnitudes of the actual forces applied to the shaker handle. Table 4.3.1 contains the means and standard deviations of the grip and push forces applied to the instrumented handle during the 45 -s force memorization period (with visual feedback) and during the 3-s force-recall period (no feedback). As indicated, subjects were able to attain the target forces at all force levels during the memorization periods of each of the experiments. At the low force target ( $15 \mathrm{~N}$ grip, $25 \mathrm{~N}$ push), the average over both experiments for grip and push memorization forces were $14.8 \mathrm{~N}$ and $24.5 \mathrm{~N}$, respectively; at 
the middle force target ( $30 \mathrm{~N}$ grip, $50 \mathrm{~N}$ push), the averages were $29.0 \mathrm{~N}$ and $48.6 \mathrm{~N}$; and at the upper force target (45 $\mathrm{N}$ grip, $75 \mathrm{~N}$ push), the averages were $43.2 \mathrm{~N}$ and $72.6 \mathrm{~N}$.

During the force recall period, subjects averaged $21.7 \mathrm{~N}$ and $31.2 \mathrm{~N}$ for grip and push force, respectively, for the low force target trials. For the medium force target trials, the recall force averages were $35.3 \mathrm{~N}$ and $53.7 \mathrm{~N}$. At the high force targets, subjects averaged $48.5 \mathrm{~N}$ grip and $78.5 \mathrm{~N}$ push.

Table 4.3.1 Grip and push forces (in Newtons) applied during the force memorization and recall periods of Experiments 1 and 2 .

\begin{tabular}{|c|c|c|c|c|c|c|c|c|c|c|}
\hline \multirow{3}{*}{ Experiment } & \multicolumn{2}{|c|}{ Target Force } & \multicolumn{4}{|c|}{ Memorization Force $\left(F_{m}\right)$} & \multicolumn{4}{|c|}{ Recall Force $\left(F_{r}\right)$} \\
\hline & \multirow{2}{*}{ Grip } & \multirow{2}{*}{ Push } & \multicolumn{2}{|c|}{ Grip } & \multicolumn{2}{|c|}{ Push } & \multicolumn{2}{|c|}{ Grip } & \multicolumn{2}{|c|}{ Push } \\
\hline & & & Mean & SD & Mean & SD & Mean & SD & Mean & SD \\
\hline \multirow{3}{*}{1} & 15 & 25 & 14.7 & 0.8 & 24.8 & 1.8 & 21.7 & 8.3 & 33.6 & 10.5 \\
\hline & 30 & 50 & 28.5 & 1.3 & 48.3 & 2.1 & 33.9 & 11.6 & 53.0 & 12.4 \\
\hline & 45 & 75 & 42.5 & 2.5 & 72.3 & 2.7 & 45.4 & 12.6 & 74.4 & 16.1 \\
\hline \multirow{3}{*}{2} & 15 & 25 & 14.9 & 0.7 & 24.3 & 1.1 & 21.6 & 10.4 & 29.6 & 9.8 \\
\hline & 30 & 50 & 29.3 & 0.9 & 48.7 & 1.3 & 36.2 & 16.1 & 54.2 & 13.4 \\
\hline & 45 & 75 & 43.6 & 1.4 & 72.8 & 1.8 & 50.5 & 19.3 & 81.2 & 18.9 \\
\hline \multirow{3}{*}{ Both } & 15 & 25 & 14.8 & 0.7 & 24.5 & 1.4 & 21.7 & 9.6 & 31.2 & 10.3 \\
\hline & 30 & 50 & 29.0 & 1.1 & 48.6 & 1.7 & 35.3 & 14.5 & 53.7 & 13.0 \\
\hline & 45 & 75 & 43.2 & 2.0 & 72.6 & 2.2 & 48.5 & 17.1 & 78.5 & 18.1 \\
\hline
\end{tabular}

\subsubsection{Recalled grip and push force distributions for Experiments $1 \& 2$}

Table 4.3.2 and Figure 4.3.1 depict the subjects' abilities to recall and reproduce the memorized grip and push forces across all conditions of both experiments. The box and whisker plots shown in Figure 4.3.1 display the distribution of the subjects' grip and push force-recall attempts at each force level. The box represents the interquartile range of the responses while the upper and lower whiskers extend to the $90^{\text {th }}$ and $10^{\text {th }}$ percentiles, respectively. The median response value for each group is also indicated in the figure. 

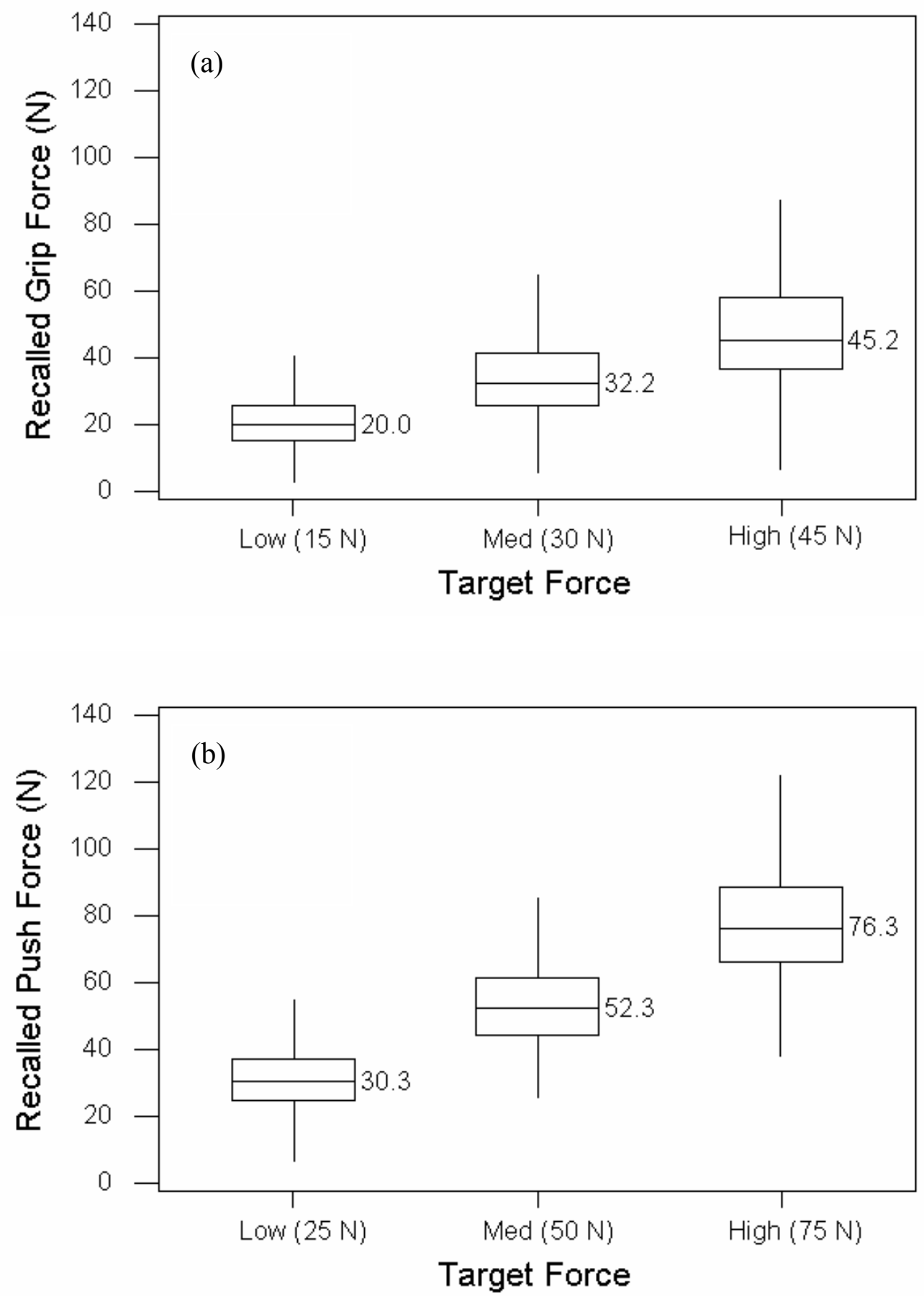

Figure 4.3.1 Distribution of recalled forces for (a) grip and (b) push by target force across all vibration exposure conditions of both experiments. 
Table 4.3.2 Distribution of recalled grip and push forces (in Newtons) across all vibration exposure conditions of both experiments.

\begin{tabular}{|c|c|c|c|c|c|c|}
\hline \multirow[b]{2}{*}{ Percentile } & \multicolumn{2}{|c|}{ Low Target Force } & \multicolumn{2}{|c|}{ Med Target Force } & \multicolumn{2}{|c|}{ High Target Force } \\
\hline & Grip $(15 N)$ & Push $(25 \mathrm{~N})$ & Grip (30 N) & Push $(50 \mathrm{~N})$ & Grip (45 N) & Push $(75 \mathrm{~N})$ \\
\hline 90th \%tile & 33.7 & 43.9 & 52.8 & 69.8 & 68.5 & 101.5 \\
\hline 75th \%tile & 25.7 & 37.0 & 41.6 & 61.3 & 57.9 & 88.6 \\
\hline Median & 20.0 & 30.3 & 32.2 & 52.3 & 45.2 & 76.3 \\
\hline 25 th $\%$ tile & 15.4 & 24.7 & 25.9 & 44.5 & 36.8 & 66.1 \\
\hline 10th \%tile & 12.1 & 20.2 & 21.6 & 38.3 & 30.7 & 58.5 \\
\hline
\end{tabular}

\subsubsection{Frequency effects found in Experiments $1 \& 2$}

The means, standard deviations, and 95\% confidence intervals for force recall in Experiments 1 and 2 combined are contained in Table 4.3.3. The vibration frequency effect is depicted in Figure 4.3.2 and Figure 4.3.3. As shown in the figure, the effect of frequency on grip forcerecall accuracy is essentially equal to its effect on push force-recall accuracy. Further, the frequency effect is most pronounced in the frequency range of $31.5 \mathrm{~Hz}$ to $63 \mathrm{~Hz}$. 
Table 4.3.3 Force recall-error means (in Newtons) at each vibration frequency evaluated in the two experiments.

\begin{tabular}{ccccc}
\hline $\begin{array}{c}\text { Vibration Freq } \\
(\mathrm{Hz})\end{array}$ & Mean & SD & \multicolumn{2}{c}{ 95\% Confidence Interval } \\
\cline { 4 - 5 } & & & Lower Bound & Upper Bound \\
\hline 0 & 3.0 & 11.8 & 1.8 & 4.2 \\
12.5 & 2.3 & 11.5 & 0.7 & 3.8 \\
16 & 4.8 & 12.8 & 3.5 & 6.0 \\
31.5 & 8.0 & 15.9 & 6.7 & 9.2 \\
40 & 8.6 & 12.9 & 7.0 & 10.2 \\
63 & 8.8 & 16.0 & 7.6 & 10.0 \\
125 & 6.8 & 14.1 & 5.9 & 7.8 \\
250 & 5.0 & 11.7 & 3.5 & 6.6 \\
\hline
\end{tabular}

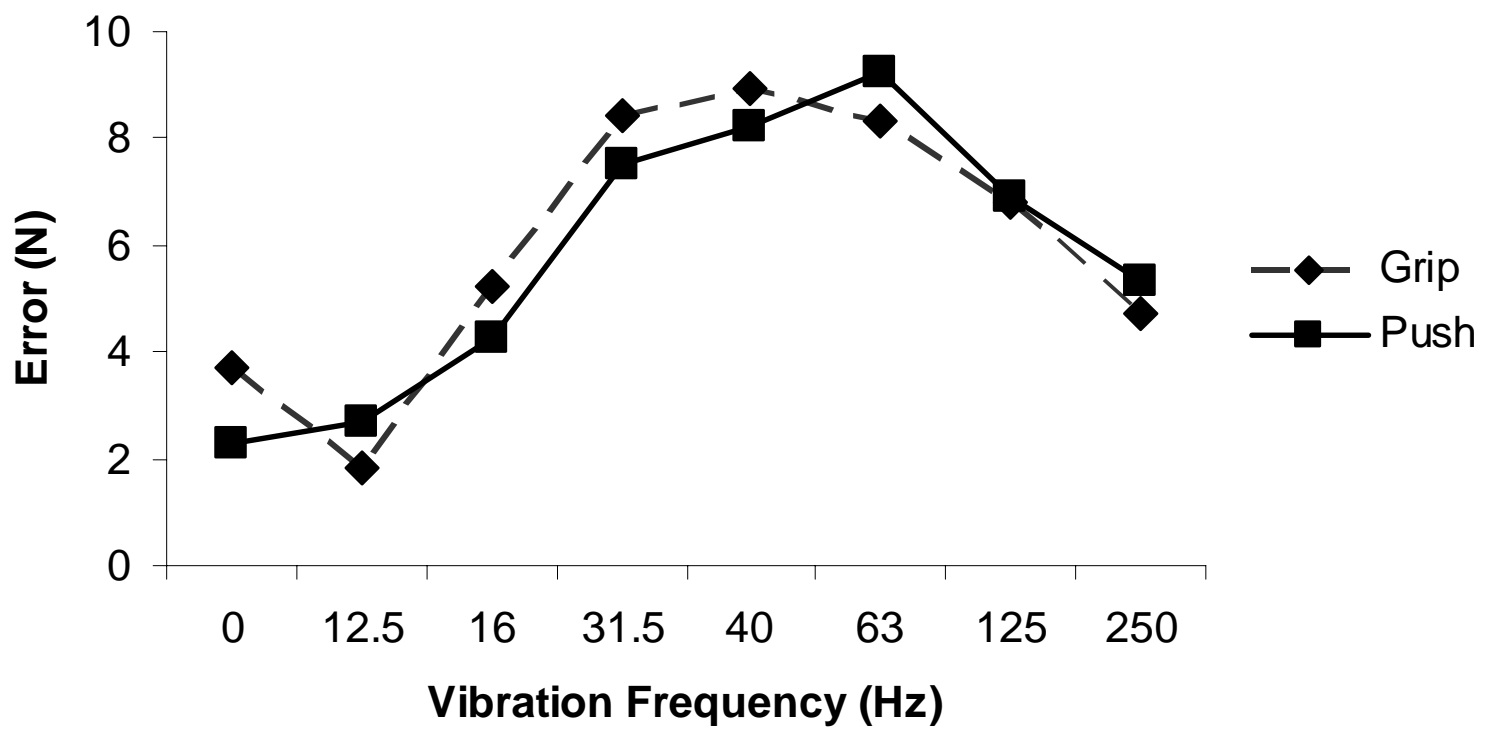

Figure 4.3.2 Grip and push force-recall error means plotted against vibration frequency across all conditions of the two experiments. 


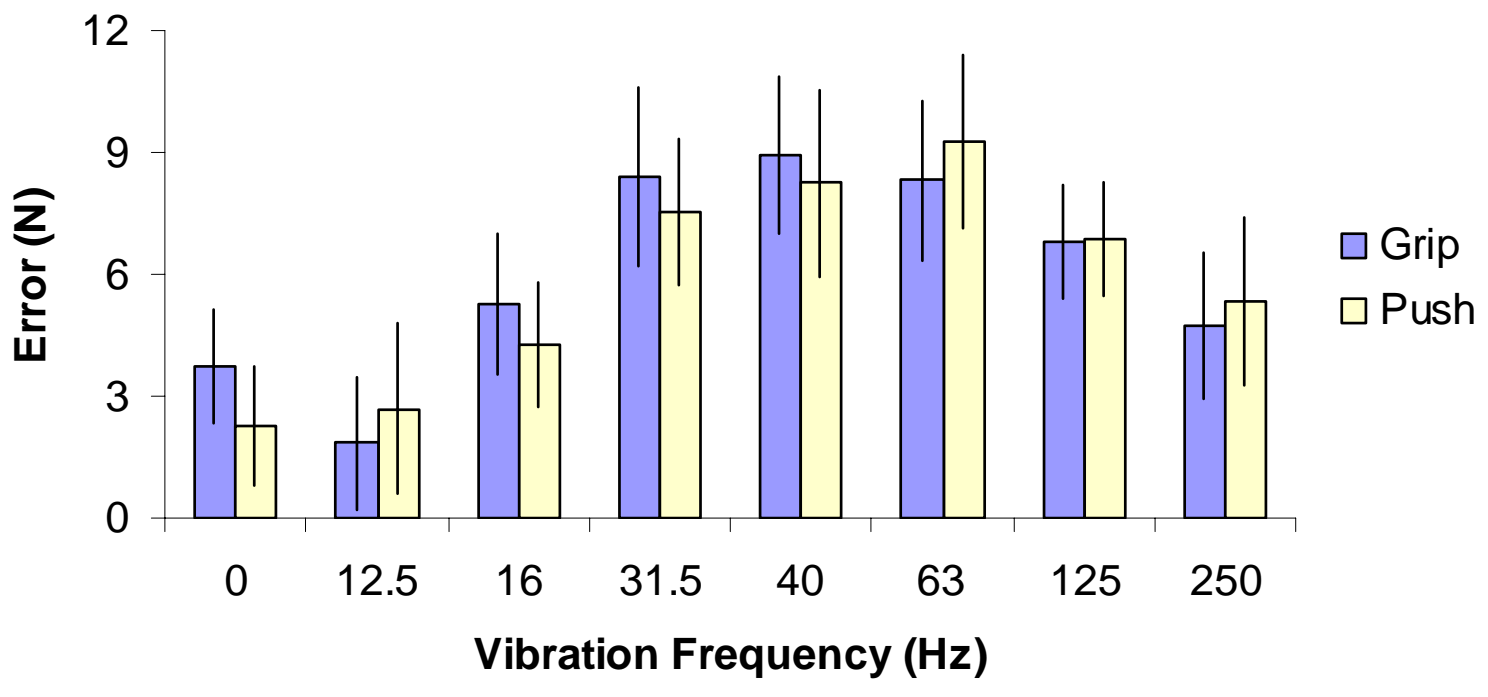

Figure 4.3.3 Grip and push force-recall error means plotted against vibration frequency for both experiments. (Y error bars $=95 \%$ C.I.) 


\section{CH A P T E R 5: D I S C U S S I O N}

\subsection{Discussion}

There is a need for alternative solutions for quantifying hand forces applied to vibrating power tools in workplace environments. Psychophysical force-recall techniques may permit assessment of these forces without the need for expensive or fragile instrumentation. An understanding of the effects of vibration and force level upon force-recall accuracy and reliability must first be explored before such methods are proposed for research or field assessments. The experiments presented here examined various combinations of handle vibration and grip and push force exposures upon the subject's ability to recall those forces. The vibration exposures and forces examined in these studies are representative of those found in typical work environments. Thus, the results of these experiments should be considered during further refinement of this psychophysical force-recall technique.

\subsubsection{Control of grip and push during the force memorization period}

The memorization portion of a trial in this study was treated as a basic tracking task. As described earlier, subjects were asked to align the needles of two dial gauges with index marks at the top of each gauge. (Please refer to Figure 3.3.4.) This visual feedback mechanism proved to be an excellent technique for fostering the control of grip and push forces. As indicated in Table 4.3.1, subjects had little trouble attaining the target grip and push forces during the memorization period. The means of actual forces applied during the force memorization period were within $3.0 \mathrm{~N}$ of the specified target force levels. Further, standard deviations were under $3.0 \mathrm{~N}$ (coefficients of variation less than 0.06 ) at all force levels for both 
grip and push. These results indicate that, by and large, subjects were able to consistently control their grip and push forces at the designated levels during force memorization.

\subsubsection{Recalled grip and push forces}

As shown in Figures 4.1.1, 4.2.1, and 4.3.1, subjects' recalled grip and push force responses were nearly evenly distributed around the median value for each exertion type and force level. Mean values were slightly higher than median values for all exertion type and force level combinations. Accordingly, the upper 'whiskers' shown in the box plots in Figures 4.1.1, 4.2.1, and 4.3.1 are slightly longer than their corresponding lower whiskers. This was to be expected considering that overestimation of force is limited only by the strength of the subject while underestimations are limited to the force range from zero to the target value.

Subjects tended to overestimate grip and push forces at all levels of target force. However, errors were small throughout the range of operationally-relevant vibration and grip force conditions of both experiments. The subjects' overestimation of grip and push forces used during the vibration exposure/memorization period is consistent with early psychophysical studies (Stevens and Mack 1959, Stevens 1960).

While humans have demonstrated a tendency for overestimating static forces in non-vibration conditions, the force overestimations realized in these two experiments may have been further influenced by vibration disruptions in the muscle afferent flow. The idea that vibration affects the perception of static force sensation is supported by the results of several studies (Cafarelli and Kostka 1981, Miall et al. 2000). Researchers have shown that vibration can excite muscle 
spindle primary endings, and to a lesser extent, spindle secondary endings, Golgi tendon organs, and Pacinian corpuscles (i.e. Burke et al. 1976, Cafarelli and Kostka 1981). The grip and push force overestimations observed in the present study lend credence to the idea that vibration-induced afferent stimulation will lead to the perception of increased intensity of intramuscular tension as described by Cafarelli and Kosta (1981).

The overestimations observed here also support the conclusions of Nowak et al. (2004). They theorized that a subject's ability to develop an awareness of the motor cortex outflow copy as well as their ability to reproduce forces based on the memory of that efferent information may be influenced by vibration. In their study, subjects repetitively lifted objects while the investigators evaluated the rate of increase of grip forces and peak grip force levels. The results indicated that the subjects could quickly memorize the grip forces necessary to perform the lifts. Subjects were also able to scale their grip forces accordingly in subsequent lifts. The results were similar when the subjects used one hand during memorization and the opposite hand to perform subsequent lifts. When muscles were vibrated during the rest period, subjects' grip force rates increased and grip force peaks were higher as compared to non-vibration trials. The researchers suggested that vibration exposure may impair the memory processes involved in grip force scaling. Moreover, they concluded that since the vibration effect also transferred to the non-vibrated hand, vibration must have affected the efferent components involved in the grip force memory process. Thus, the vibration effect on the efferent memory may affect force-recall accuracy. This conclusion may at least partially explain the grip and push force overestimations realized in both experiments of this study. 


\subsubsection{Vibration frequency effects}

The results of this study indicate that vibration frequency is a significant influence on forcerecall accuracy. Overestimations were significantly higher during vibration exposures in the range from $31.5 \mathrm{~Hz}$ to $63 \mathrm{~Hz}$. Errors at the $125 \mathrm{~Hz}$ level were also found to be statistically significant in Experiment 1 and suggestively significant in Experiment 2. As discussed in Chapter 2, these frequencies fall within the resonance frequency ranges for the hand-arm system $(25-63 \mathrm{~Hz})$ and the fingers $(100-250 \mathrm{~Hz})$, respectively (Sörensson and Lundström 1992, Kihlberg 1995, ISO 1998, Dong et al. 2004a,b). At or near resonance, bio-mechanical systems and sub-systems are more responsive to vibration stimuli. Thus, it follows that vibrationinduced disruptions in force memorization and recall processes are expected to be greater in these vibration frequency ranges.

Radwin et al. (1987) reported, using a comparable stimulus methodology, that responses attributed to the tonic vibration reflex (TVR) were greatest at $40 \mathrm{~Hz}$; the frequency which demonstrated the greatest error in the force-recall trials of Experiment 1. In Experiment 2, the greatest errors occurred at $31.5 \mathrm{~Hz}$ and $63 \mathrm{~Hz}$. Given that the greatest force-recall errors occurred at and around $40 \mathrm{~Hz}$ in these two experiments, and because the experiments were not designed to rule out nor confirm the contribution of TVR, the potential of TVR contribution to force-recall errors in this study cannot be ruled out. However, as noted in Chapter 2, in most early TVR experiments, the reflex action was initiated by directly applying vibration to the muscle tendon. In fact, in their early study of this reflex phenomenon, Hagbarth and Eklund (1965) observed that the reflex action was not normally induced unless vibration was directly applied to the muscle tendons. Moreover, any reflex action was limited to the muscles whose 
tendons were directly stimulated. Thus, it is unlikely that a voluntary power grip or palm push motor function could be affected by a TVR action initiated through hand-transmitted vibration. Furthermore, TVR studies have shown that even when vibration stimulus is directly applied to a muscle tendon, subjects anticipating a vibration stimulus can counteract or prevent the reflex action entirely, especially if visual feedback of limb position or muscle force is provided (Hagbarth and Eklund 1965, de Gail et al. 1966).

\subsubsection{Vibration magnitude effects}

Unlike Experiment 1, Experiment 2 examined different levels of vibration magnitude. As expected, the second experiment reveled vibration magnitude to be a significant influential factor on force-recall accuracy. Errors were greater during trials with higher vibration magnitude exposures than during low-magnitude or zero-vibration exposures. Exposures to higher vibration magnitudes induces more stress into finger, hand, and arm tissues resulting in an increase in afferent nervous activity. As discussed above, previous research has suggested that such an increase will lead to greater overestimations of grip and push forces (Cafarelli and Kostka 1981). Likewise, disruptions in efferent memory as described by Nowak et al. (2004) can be expected to increase with the increase of vibration intensity. Hence, it follows that force-recall accuracy should suffer as vibration intensity increases.

The vibration magnitude by vibration frequency interaction was found to be significant. As presented in section 4.2.7, the vibration magnitude effect was considerably more pronounced at $31.5 \mathrm{~Hz}$ and $63 \mathrm{~Hz}$ than at the other exposure frequencies. This is not surprising considering that the effects of vibration would be expected to be greater with vibration exposure 
frequencies near system resonance when compared to vibration exposures outside the resonance frequency range. As discussed earlier, the range of hand-arm system resonance has been reported to extend from $25 \mathrm{~Hz}$ to $63 \mathrm{~Hz}$ (Kihlberg 1995, ISO 1998, Dong et al. 2004a). Thus, the observed interaction effect falls perfectly in line with previously reported findings.

Vibration magnitude also interacted significantly with exertion type in Experiment 2. High magnitudes of vibration adversely affected push force-recall accuracy considerably more than grip force-recall accuracy. The results of a recent study by Ebied et al. (2004) indicate that muscle afferents are more influential than cutaneous afferent feedback during isometric force production. While the mechanisms of grip and push force recall are not fully known, it seems reasonable to speculate that due to the contributions of finger afferents, grip force perception and recall would involve a higher percentage of cutaneous signals than would push force recall. Cutaneous afferent contributions to push force perception and recall would be limited to the palm area. That is to say, push force recall may rely almost entirely on a combination of efferent memory and muscle afferent signals rather than cutaneous afferents. Thus, because high magnitude vibration induces increased afferent nervous activity in muscle tissue, this level of vibration magnitude would have a greater influence on push force perception than on grip force perception. 


\subsubsection{Force level effects}

In Experiment 1, force level was found to be significant. For both grip and push, the mean force-recall error decreased as force level increased (see Figure 4.1.6). However, this trend was not apparent in Experiment 2. In the second experiment, grip force-recall error means remained steady across all three force levels (see Figure 4.2.10). On the other hand, push force-recall errors were higher at the highest force level than at the other two force levels. These basic trends are evident across both genders and across both vibration magnitudes.

At first glance, as regards force level effects, the results of the two experiments seem to contradict one another. However, an evaluation of force recall accuracy in terms of percentage reveals a fairly consistent pattern. Figure 5.1.1 and Figure 5.1.2 illustrate force recall accuracy as a percentage of target force for each relative force level for Experiments 1 and 2, respectively. As shown in both figures, there is a general trend for percentage error to decrease as the force level increases. These results are consistent with previous research (Lowe 1995). 


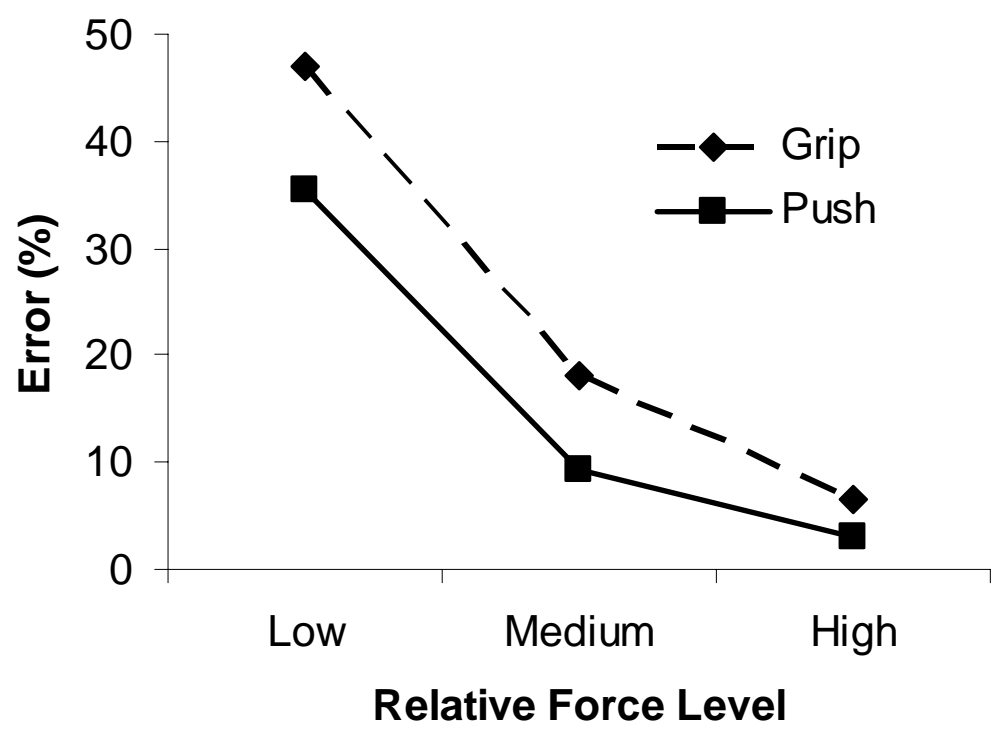

Figure 5.1.1 Force-recall error expressed as a percentage of target force for grip and push plotted against relative force level for Experiment 1.

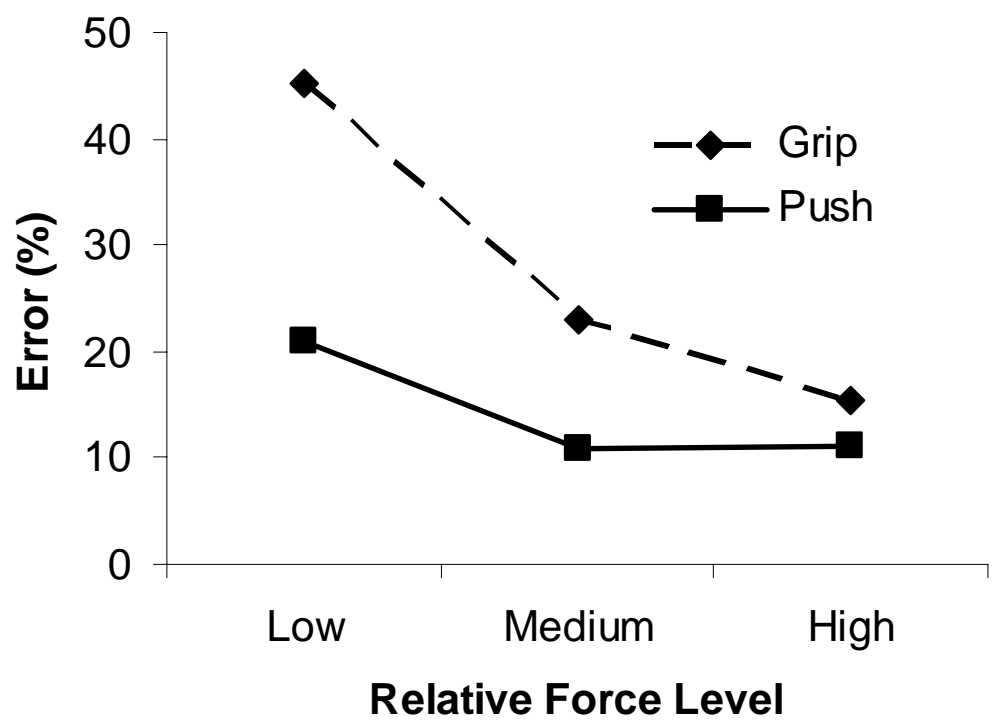

Figure 5.1.2 Force-recall error expressed as a percentage of target force for grip and push plotted against relative force level for Experiment 2. 


\subsubsection{Gender effect}

In the second experiment, females were found to be significantly more accurate than males across all conditions of the experiment (refer to Table 4.2.15). Furthermore, a suggestively significant gender by exertion type interaction emerged. As was the case in Experiment 1 (all male subjects), the Experiment 2 male subjects' mean force-recall errors were nearly equal for grip and push. For females, however, the mean recalled grip force error exceed that of recalled push force error. These phenomena are likely functions of the subject's relative grip and push strength.

On average, males are stronger than females when comparing actions that involve the upper extremities such as gripping, pushing, and pulling (Kumar et al. 1995, Imrhan 1999). In Experiment 2, the target grip and push forces were the same for male and female subjects. While this experiment did not include tests for subject strength, the target grip and push forces were likely nearer to the maximal forces for the female subjects than to those of the male subjects. Lowe (1995) found that grip force-recall accuracy varies with the magnitude of the target force. As described in Chapter 2, Lowe's subjects were asked to match four levels of target grip force $(20 \%, 35 \%, 50 \%$, and $65 \% \mathrm{MVC})$. The analysis indicated that grip force level is a significant factor. The subjects were more accurate when attempting to match the $50 \%$ and $65 \%$ MVC targets than with the $20 \%$ and $35 \%$ targets. If the female subjects used in Experiment 2 were indeed tasked with greater force:MVC ratios than their male counterparts, then the results of this study are consistent with Lowe's (1995). 


\subsubsection{Test-retest reliability}

In each of the two experiments, the average recalled force error was less for the second test session than for the first test session. This increase in accuracy was found to be statisticallysignificant in Experiment 2 but was not significant in Experiment 1. It was previously noted that the same subject instruction set was used during Experiments 1 and 2 (see Appendix D). The instruction set was also the same for Day 1 and Day 2. Further, no feedback was given to the subjects regarding their performance during any individual trial or test session. No attempt was made to 'train' the subjects. The mechanisms by which the subjects improved their performances were not identified in this study.

While recalled force errors decreased from the first session trials to the second session trials in both experiments, an examination of the actual grip and push forces produced during the recall attempts (as opposed to recalled force error) revealed strong test-retest reliability. For push force, Pearson correlation coefficients for the Day 1 and Day 2 results were significant (twotailed, 0.01 level) for all 32 subjects employed in the two experiments. The same was true for grip force with the exception of one male subject in Experiment 2. These correlations are indicative of strong test-retest reliability. 


\subsection{Conclusions}

The primary conclusion of this research is that this force-recall technique shows promise as an alternative to expensive and fragile force-sensing instrumentation, and it should be further studied as a method for characterizing grip and push forces in field and laboratory settings. Subjects tended to overestimate grip and push forces, but overall, recalled grip and push force errors were relatively small over the entire range of operationally-relevant hand-handle coupling forces and vibration exposure conditions. This force-recall technique demonstrated strong test-retest reliability. Once all relevant influential factors have been identified and characterized, those factors can be accounted for in enhanced force-recall methods that can be incorporated into a variety of exposure assessment applications.

This research has clearly identified three important factors that significantly affect the accuracy of recalled grip and push forces. Those three factors are summarized here:

1. Vibration frequency - Recalled grip and push force errors were highest when vibration exposures were in the frequency range from $31.5 \mathrm{~Hz}$ to $63 \mathrm{~Hz}$. The resonance frequency of the human hand-arm system typically falls within this same frequency range. 
2. Vibration magnitude - Errors were significantly greater at the higher magnitudes of vibration exposure in this study. Significant interactions were also revealed between vibration magnitude and vibration frequency and between vibration magnitude and exertion type. At higher vibration magnitudes, vibration energy penetrates deeper into finger, hand, and arm tissues resulting in the activation of greater numbers of mechanoreceptors. This increase in afferent nervous activity likely contributes to greater overestimations of grip and push forces. Moreover, disruptions in efferent memory can be expected to increase with the increase of vibration magnitude. These two sources of disruption likely combine to cause a decrease in force-recall accuracy.

3. Gender/Relative force level - Females produced recall forces closer to the target forces than their male counterparts. This phenomena is likely a function of the relative strength of the subject. By and large, males are stronger than females. Previous studies have shown that subjects are more accurate when matching forces closer to their maximal voluntary contraction forces. Thus, because both sexes used the same target forces, females would be expected to be more accurate in their force recall attempts.

\subsection{Recommendations for future studies}

Before this psychophysical force-recall technique is employed in the field, several refinements will have to be made. All important factors that affect force-recall accuracy will have to be identified and accounted for via weighting functions or other appropriate means. This study identified some important factors that contribute to force-recall accuracy, but further 
experiments are necessary before this technique can reach its full potential. Several suggestions for future research are outlined below.

- Biodynamic response - In this study, the frequency range with the greatest force-recall errors coincides with the frequency range associated with hand-arm system resonance. Vibration-induced disruptions in force perception are likely to be particularly pronounced at the resonance frequency. It is also known that mechanical systems, including the hand-arm system, exhibit particularly high impedance values at resonance frequencies. Researchers have developed laboratory techniques for measuring the mechanical impedance of human finger-hand-arm systems coupled to instrumented handles (see Dong et al., 2004a,b, 2005a,b). Therefore, distinct relationships between an individual's biodynamic response to a particular vibration exposure and that individual's force-recall response at that exposure can be thoroughly examined. Further, because a subject's biodynamic response is related to his/her physical attributes, it may be possible to predict a range of force-recall accuracy based upon certain aspects of an individual's anthropometry and/or measurements of other physical properties.

- Force level effects - In each of these experiments, force recall accuracy, expressed as a percentage of the target force, increased as the target force increased. Furthermore, female subjects were shown to be more accurate than male subjects; and this phenomenon is likely associated with the relative strength of the subject rather than gender. A force recall study with target forces based on percentages of each subject's maximal voluntary contraction (MVC) could clear up this issue. 
- Delay between exposure and force recall - The amount of time between the vibration exposure/force memorization period and the force-recall attempt is another potential influence on force-recall accuracy. In the first experiment, this factor was examined at two levels: $10 \mathrm{~s}$ and $20 \mathrm{~s}$. This factor was not found to be statistically significant, but it was suggestively significant. It could be that the two levels examined are too close to cause a meaningful difference. An examination of additional levels of delay may provide important information. Further, activities or exposures between the force memorization period and the force-recall period may need to be controlled or accounted for. For example, an activity such as tool relocation just prior to the force-recall attempt may interfere with the force memory process and lead to additional force-recall errors. This is an aspect that may require close examination.

- Glove use - The use of standard work gloves or anti-vibration gloves may diminish the amount of vibration energy entering a subject's hand-arm system and, in turn, reduce vibration-induced disruptions during force memorization and recall. A recent pilot study by McDowell et al. (2004) supports that hypothesis. In their study, subjects were more accurate during trials when they operated a vibrating tool with gloves rather than with bare their hands. Because glove use is common in many work environments where vibrating hand tools are used, glove use during force memorization and recall requires further investigation. 
- Training - In this study, subjects were encouraged to do their best when memorizing and recalling grip and push forces. Beyond that encouragement, however, no attempts were made toward the development of ways to facilitate more accurate results. The instruction set used in these experiments did not vary. The instructions were designed to produce consistent results; they were not intended to maximize accuracy. The subjects were not provided with any feedback regarding their force-recall performance. Modification of the instruction set and the introduction of feedback during practice trials would undoubtedly improve force-recall accuracy. 


\section{BIBLIOGRAPHY}

ANSI. 1986, ANSI S3.34: Guide for the Measurement and Evaluation of Human Exposure to Vibration Transmitted to the Hand, (American National Standards Institute (ANSI), New York).

Bao, S, and Silverstein, B. 2005, Estimation of Hand Forces in Ergonomic Job Evaluations. Ergonomics, 48 (3), 288-301.

Borg, GAV. 1962, Physical Performance and Perceived Exertion. (Copenhagen: Ejnar Munksgaard).

Borg, GAV. 1970, Perceived Exertion as an Indicator of Somatic Stress. Scandinavian Journal of Rehabilitative Medicine, 2, 92-98.

Borg, GAV. 1982, Psychophysical Bases of Perceived Exertion. Medicine and Science in Sports and Exercise, 14 (5), 377-381.

Borg, GAV. 1990, Psychophysical Scaling with Applications in Physical Work and the Perception of Exertion. Scandinavian Journal of Work, Environment \& Health, 16 (Supplement 1), 55-58.

Bovenzi, M. 1998, Exposure-Response Relationship in the Hand-Arm Vibration Syndrome: An Overview of Current Epidemiology Research. International Archives of Occupational and Environmental Health, 71, 509-519.

Brammer, AJ. 1982, Threshold Limits for Hand-Arm Vibration Exposure Throughout the Workday. In AJ Brammer and W Taylor (Eds.) Vibration Effects on the Hand and Arm in Industry. (Wiley and Sons, New York).

Burgess, PR, and Jones, LF. 1997, Perceptions of Effort and Heaviness During Fatigue and During the Size-Weight Illusion. Somatosensory and Motor Research, 14 (3), 189202.

Burke, D, Hagbarth, K-E, Löfstedt, L, and Wallin, BG. 1976, The Responses of Human Muscle Spindle Endings to Vibration During Isometric Contraction. Journal of Physiology, 261, 695-711.

Cafarelli, E, and Bigland-Ritchie, B. 1979, Sensation of Static Forces in Muscles of Different Length. Experimental Neurology, 65, 511-525.

Cafarelli, E, and Kostka, CE. 1981, Effect of Vibration on Static Force Sensation in Man. Experimental Neurology, 74, 331-340.

Cannon, LJ, Bernacki, EJ, and Walter, SD. 1981, Personal and Occupational Factors Associated with Carpal Tunnel Syndrome. Journal of Occupational Medicine, 23 (4), 255-258. 
Carlsöö, S. 1986, With What Degree of Precision Can Voluntary Static Muscle Force Be Repeated? Scandinavian Journal of Rehabilitation Medicine, 18 (1), 1-3.

Carlsöö, S, and Mayr, J. 1974, A Study of the Load on Joints and Muscles in Work with a Pneumatic Hammer and a Bolt Gun. Work-Environment-Health, 11 (1), 32-38.

Chadwick, EKJ, and Nicol, AC. 2001, A Novel Force Transducer for the Measurement of Grip Force. Journal of Biomechanics, 34 (1), 125-128.

Ciriello, VM, Snook, SH, Blick, AC, and Wilkinson, PL. 1990, The Effects of Task Duration on Psychophysically-Determined Maximum Acceptable Weights and Forces. Ergonomics, 33 (2), 187-200.

de Gail, P, Lance, JW, and Neilson, PD. 1966, Differential Effects on Tonic and Phasic Reflex Mechanisms Produced by Vibration of Muscles in Man. Journal of Neurology, Neurosurgery, and Psychiatry, 29, 1-11.

Dong, RG, McDowell, TW, and Welcome, DE. 2005a, Biodynamic Response at the Palm of the Human Hand Subjected to a Random Vibration. Industrial Health, 43 (1), 241255.

Dong, RG, Schopper, AW, McDowell, TW, Welcome, DE, Wu, JZ, Smutz, WP, Warren, C, and Rakheja, S. 2004a, Vibration Energy Absorption (VEA) in Human Fingers-HandArm System. Medical Engineering and Physics, 26 (6), 483-492.

Dong, RG, Welcome, DE, McDowell, TW, and Wu, JZ. 2004b, Biodynamic Response of Human Fingers in a Power Grip Subjected to a Random Vibration. Journal of Biomechanical Engineering, 126 (4), 447-457.

Dong, RG, Wu, JZ, McDowell, TW, Welcome, DE, and Schopper, AW. 2005b, Distribution of Mechanical Impedance at the Fingers and the Palm of the Human Hand. Journal of Biomechanics, 38 (5), 1165-1175.

Ebied, AM, Kemp, GJ, and Frostick, SP. 2004, The Role of Cutaneous Sensation in the Motor Function of the Hand. Journal of Orthopaedic Research, 22 (4), 862-866.

Eisler, H. 1962, Subjective Scale of Force for a Large Muscle Group. Journal of Experimental Psychology, 64 (3), 253-257.

Feldman, AG, and Latash, ML. 1982, Afferent and Efferent Components of Joint Sense; Interpretation of Kinaesthetic Illusion. Biological Cybernetics, 42 (3), 205-214.

Gandevia, SC, Macefield, G, Burke, D, and McKenzie, DK. 1990, Voluntary Activation of Human Motor Axons in the Absence of Muscle Afferent Feedback. The Control of the Deafferented Hand. Brain, 113, 1563-1581.

Garg, A, Mital, A, and Asfour, SS. 1980, A Comparison of Isometric Strength and Dynamic Lifting Capability. Ergonomics, 23 (1), 13-27. 
Gemne, G, and Taylor, W. 1983, Foreword: Hand-Arm Vibration and the Central Autonomic Nervous System. Journal of Low Frequency Noise and Vibration, Special Volume, $1-12$.

Gooch, JL, and Randle, J. 1993, Force Perception before and after Maximal Voluntary Contraction. Perceptual and Motor Skills, 76 (2), 399-402.

Goodwin, GM, McCloskey, DI, and Matthews, PBC. 1972, The Contribution of Muscle Afferents to Kinaesthsia Shown by Vibration Induced Illusions of Movement and by the Effects of Paralysing Joint Afferents. Brain, 95 (4), 705-748.

Griffin, MJ. 1990, Handbook of Human Vibration. (London: Academic Press).

Griffin, MJ. 1997, Measurement, Evaluation, and Assessment of Occupational Exposures to Hand-Transmitted Vibration. Occupational \& Environmental Medicine, 54 (2), 73-89.

Griffin, MJ, and Bovenzi, M. 2001, The Diagnosis of Disorders Caused by HandTransmitted Vibration: Southampton Workshop 2000. International Archives of Occupational and Environmental Health, 75 (1-2), 1-5.

Gurram, R, Rakheja, S, and Gouw, G. 1995, A Study of Hand Grip Pressure Distribution and EMG of Finger Flexor Muscles under Dynamic Loads. Ergonomics, 38 (4), 684-699.

Hagbarth, K-E, and Eklund, G. 1965, Motor Effects of Vibratory Muscle Stimuli in Man. In R Granit (Ed.) Muscular Afferents and Motor Control. Proceedings of Nobelsymposium I. (John Wiley \& Sons, New York), 177-186.

Hammarskjöld, E, Ekolm, J, and Harms-Ringdahl, K. 1989, Reproducibility of Work Movements with Carpenters' Hand Tools. Ergonomics, 32 (8), 1005-1018.

Hammarskjöld, E, Harms-Ringdahl, K, and Ekolm, J. 1990, Shoulder-Arm Muscular Activity and Reproducibility in Carpenters' Work. Clinical Biomechanics, 5 (2), 81-87.

Hartung, E, Dupuis, H, and Scheffer, M. 1993, Effects of Grip and Push Forces on the Acute Response of the Hand-Arm System under Vibrating Conditions. International Archives of Occupational and Environmental Health, 64 (6), 463-467.

Heide, J, and Molbech, S. 1973, Influence of after-Movement on Muscle Memory Following Isometric Muscle Contraction. Ergonomics, 16 (6), 787-796.

Henningsen, H, Ende-Henningsen, B, and Gordon, AM. 1995a, Asymmetric Control of Bilateral Isometric Finger Forces. Experimental Brain Research, 105 (2), 304-311.

Henningsen, H, Ende-Henningsen, B, and Gordon, AM. 1995b, Contribution of Tactile Afferent Information to the Control of Isometric Finger Forces. Experimental Brain Research, 105 (2), 312-317. 
Imrhan, SN. 1999, Push-Pull Force Limits. In W Karwowski and WS Marras (Eds.) The Occupational Ergonomics Handbook. (CRC Press, Boca Raton, FL).

ISO. 1996, ISO 10819, 1996: Mechanical Vibration and Shock -- Hand-Arm Vibration -Method for the Measurement and Evaluation of the Vibration Transmissibility of Gloves at the Palm of the Hand. ISO 10819:1996(E), (International Organization for Standardization, Geneva, Switzerland).

ISO. 1998, ISO 10068, 1998: Mechanical Vibration and Shock -- Free, Mechanical Impedance of the Human Hand-Arm System at the Driving Point. ISO 10068:1998, (International Organization for Standardization, Geneva, Switzerland).

ISO. 2001, ISO 5349-1, 2001: Mechanical Vibration -- Measurement and Evaluation of Human Exposure to Hand-Transmitted Vibration -- Part 1: General Requirements. ISO 53491:2001, (International Organization for Standardization, Geneva, Switzerland).

ISO. 2005, ISO/DIS 15230, 2005: Mechanical Vibration and Shock - Coupling Forces at the Machine-Man Interface for Hand-Transmitted Vibration, (International Organization for Standardization, Geneva, Switzerland).

Johansson, RS, and Vallbo, Å. 1983, Tactile Sensory Coding in the Glabrous Skin of the Human Hand. Trends in Neurological Science, 6, 27-32.

Jung, M-C, and Hallbeck, MS. 2002, The Effect of Wrist Position, Angular Velocity, and Exertion Direction on Simultaneous Maximal Grip Force and Wrist Torque under the Isokinetic Conditions. International Journal of Industrial Ergonomics, 29 (3), 133-143.

Karwowski, W, Ayoub, MM, Alley, LR, and Smith, JL. 1984, Fuzzy Approach in Psychophysical Modeling of Human Operator-Manual Lifting System. Fuzzy Sets and Systems, 14 (1), 65-76.

Karwowski, W, Shumate, C, Yates, JW, and Pongpatana, N. 1992, Discriminability of Load Heaviness: Implications for the Psychophysical Approach to Manual Lifting. Ergonomics, 35 (7/8), 729-744.

Kaulbars, U. 1996, Measurement and Evaluation of Coupling Forces When Using Hand-Held Power Tools. Central European Journal of Public Health, 4 (1), 57-58.

Kihlberg, S. 1995, Biodynamic Response of the Hand-Arm System to Vibration from an Impact Hammer and a Grinder. International Journal of Industrial Ergonomics, 16 (1), $1-8$.

Kihlberg, S, and Hagberg, M. 1997, Hand-Arm Symptoms Related to Impact and Nonimpact Hand-Held Power Tools. International Archives of Occupational and Environmental Health, 69 (4), 282-288.

Kumar, S, Narayan, Y, and Bacchus, C. 1995, Symmetric and Asymmetric Two-Handed PullPush Strength of Young Adults. Human Factors, 37 (4), 854-865. 
Kumar, S, and Simmonds, M. 1994, The Accuracy of Magnitude Production of Submaximal Precision and Power Grips and Gross Motor Efforts. Ergonomics, 37 (8), 1345-1353.

Kumar, S, Simmonds, M, and Lechelt, D. 1994, Maximal and Graded Effort Perception by Young Females in Stoop Lifting, Hand-Grip and Finger Pinch Activity with Comparisons to Males. International Journal of Industrial Ergonomics, 13 (1), 3-13.

Lacourse, MG, and Morris, HH. 1991, Discrimination of Weight During Rapid Goal-Directed Movements of the Lower Arm. Journal of Human Movement Studies, 20 (2), 71-84.

Lafargue, G, Paillard, J, Lamarre, Y, and Sirigu, A. 2003, Production and Perception of Grip Force without Proprioception: Is There a Sense of Effort in Deafferented Subjects? European Journal of Neuroscience, 17 (12), 2741-2749.

Legg, SJ, and Myles, WS. 1981, Maximum Acceptable Repetitive Lifting Workloads for an 8-Hour Work-Day Using Psychophysical and Subjective Rating Methods. Ergonomics, 24 (12), 907-916.

Lowe, BD. 1995, Repeatability of Magnitude Production in Isometric, Hand-Grip Force Estimation: A Working-Memory Approach. Perceptual and Motor Skills, 80 (2), 659-667.

Lundström, R. 1984, Local Vibrations - Mechanical Impedance of the Human Hand's Glabrous Skin. Journal of Biomechanics, 17, 137-144.

Lundström, R, and Johansson, RS. 1986, Acute Impairment of the Sensitivity of Skin Mechanoreceptive Units Caused by Vibration Exposure of the Hand. Ergonomics, 29 (5), 687-698.

Matthews, PBC. 1982, Where Does Sherrington's 'Muscular Sense' Originate? Muscles, Joints, Corollary Discharges? Annual Review of Neuroscience, 5, 189-218.

McCloskey, DI. 1974, Muscular and Cutaneous Mechanisms in the Estimation of the Weights of Grasped Objects. Neuropsychologia, 12, 513-520.

McCloskey, DI. 1981, Chapter 32: Corollary Discharges: Motor Commands and Perception Handbook of Physiology Vol. 2 - The Nervous System. (American Physiological Society, Bethesda, Maryland, USA), 1415-1447.

McCloskey, DI, Cross, MJ, Honner, R, and Potter, EK. 1983, Sensory Effects of Pulling or Vibrating Exposed Tendons in Man. Brain, 106 (1), 21-37.

McDowell, TW, Dong, RG, and Welcome, DE. 2004, A Pilot Study of Glove Effects on a Force Matching Method Conference Program \& Preconference Proceedings of the 10th International Conference on Hand-Arm Vibration, Las Vegas, NV, USA), 83-84.

McGorry, RW. 2001, A System for the Measurement of Grip Forces and Applied Moments During Hand Tool Use. Applied Ergonomics, 32 (3), 271-279. 
Miall, RC, Ingram, HA, Cole, JD, and Gauthier, GM. 2000, Weight Estimation in a 'Deafferented' Man and in Control Subjects: Are Judgments Influenced by Peripheral or Central Signals? Experimental Brain Research, 133 (4), 491-500.

Montgomery, DC. 2001, Design and Analysis of Experiments. 5th Ed. (New York: John Wiley \& Sons).

NIOSH. 1989, Criteria for a Recommended Standard: Occupational Exposure to Hand-Arm Vibration, NIOSH Publication 89-106. (Cincinnati, OH: U.S. Department of Health and Human Services, National Institute for Occupational Safety and Health).

NIOSH. 1997, Musculoskeletal Disorders and Workplace Factors: A Critical Review of Epidemiologic Evidence for Work-Related Musculoskeletal Disorders of the Neck, Upper Extremity, and Low Back, NIOSH Publication 97-141. (Cincinnati, OH: U.S. Department of Health and Human Services, National Institute for Occupational Safety and Health).

Nowak, DA, Glasauer, S, and Hermsdorfer, J. 2003, How Predictive Is Grip Force Control in the Complete Absence of Somatosensory Feedback? Brain, 127, 182-192.

Nowak, DA, Rosenkranz, K, Hermsdorfer, J, and Rothwell, J. 2004, Memory for Fingertip Forces: Passive Hand Muscle Vibration Interferes with Predictive Grip Force Scaling. Experimental Brain Research, 156 (4), 444-450.

Pearson, ES, and Hartley, HO. 1972, Biometrika Tables for Statisticians. (Cambridge: Cambridge University Press).

Pytel, JL, and Kamon, E. 1981, Dynamic Strength Test as a Predictor for Maximal and Acceptable Lifting. Ergonomics, 24 (9), 663-672.

Radwin, RG, Armstrong, TJ, and Chaffin, DB. 1987, Power Hand Tool Vibration Effects on Grip Exertions. Ergonomics, 30 (5), 833-855.

Radwin, RG, Masters, GP, and Lupton, FW. 1991, A Linear Force-Summing Hand Dynamometer Independent of Point of Application. Applied Ergonomics, 22 (5), 339-345.

Radwin, RG, and Yen, TY. 1999, Force Dynamometers and Accelerometers. In W Karwowski and WS Marras (Eds.) The Occupational Ergonomics Handbook. (CRC Press, Boca Raton, FL).

Riedel, S. 1995, Consideration of Grip and Push Forces for the Assessment of Vibration Exposure. Central European Journal of Public Health, 3 (Supplement), 139-141.

Schoenmarklin, RW, and Marras, WS. 1989, Effects of Handle Angle and Work Orientation on Hammering: II. Muscle Fatigue and Subjective Ratings of Body Discomfort. Human Factors, 31 (4), 413-420. 
Silverstein, BA, Fine, LJ, and Armstrong, TJ. 1987, Occupational Factors and the Carpal Tunnel Syndrome. American Journal of Industrial Medicine, 11 (3), 343-358.

Snook, SH. 1978, The Design of Manual Handling Tasks. Ergonomics, 21 (12), 963-985.

Sörensson, A, and Lundström, R. 1992, Transmission of Vibration to the Hand. Journal of Low Frequency Noise and Vibration, 11, 14-22.

Starck, J, Jussi, P, and Ilmari, P. 1990, Physical Characteristics of Vibration in Relation to Vibration-Induced White Finger. American Industrial Hygiene Association Journal, 51 (4), 179-184.

Stevens, JC, and Mack, JD. 1959, Scales of Apparent Force. Journal of Experimental Psychology, 58 (5), 405-413.

Stevens, SS. 1957, On the Psychophysical Law. Psychological Review, 64, 153-181.

Stevens, SS. 1960, The Psychophysics of Sensory Function. American Scientist, 48, 226-253.

Stevens, SS. 1986, Psychophysics: Introduction to Its Perceptual, Neural, and Social Prospects. (New Brunswick, NJ, USA: John Wiley \& Sons).

Taylor, W, and Brammer, AJ. 1982, Vibration Effects on the Hand and Arm in Industry: An Introduction and Review. In AJ Brammer and W Taylor (Eds.) Vibration Effects on the Hand and Arm in Industry. (John Wiley \& Sons, New York).

Ulin, SS, Armstrong, TJ, Snook, SH, and Monroe-Keyserling, W. 1993, Examination of the Effect of Tool Mass and Work Postures on Perceived Exertion for a Screw Driving Task. International Journal of Industrial Ergonomics, 12 (1-2), 105-115.

Ulin, SS, Ways, CM, Armstrong, TJ, and Snook, SH. 1990, Perceived Exertion and Discomfort Versus Work Height with a Pistol-Shaped Screwdriver. American Industrial Hygiene Association Journal, 51 (11), 588-594.

Verrillo, RT. 1975, Cutaneous Sensation. In B Scharf(Ed.) Experimental Sensory Psychology. (Scott, Foresman and Company, Glenview. IL, USA), 151-184.

Vincent, HK, Carlson, C, Hyatt, JPK, Yihua, L, and Vincent, KR. 2000, Alterations in Bilateral Force Judgment Following Strenuous Eccentric Exercise. Research Quarterly for Exercise and Sport, 71 (4), 340-348.

Wang, M-JJ, Liu, C-M, and Shih, Y-C. 1991, A Method for Determining the Difference Threshold of Judging Weight Difference in Material Handling. International Journal of Industrial Ergonomics, 8 (4), 335-343.

Wangenheim, M, Carlsöö, S, Nordgren, B, and Linroth, K. 1986, Perception of Efforts in Working Postures. Upsala Journal of Medical Sciences, 91 (1), 53-66. 
Wasserman, DE. 1998, The Control of Hand-Arm Vibration Exposure. In PL Pelmear and DE Wasserman (Eds.) Hand-Arm Vibration: A Comprehensive Guide for Occupational Health Professionals. (OEM Press, Beverly Farms, MA).

Wasserman, DE, Wasserman, J, and Ahn, JI. 2001, Instrumentation for Measuring Coupling Forces of Hand-Held Tools. Journal of Sound and Vibration, 35 (7), 22-25.

Watson, JDG, Colebatch, JG, and McCloskey, DI. 1984, Effects of Externally Imposed Elastic Loads on the Ability to Estimate Position and Force. Behavioural Brain Research, 13 (3), 267-271.

Welcome, DE, Rakheja, S, Dong, RG, Wu, JZ, and Schopper, AW. 2004, An Investigation on the Relationship between Grip, Push, and Contact Forces Applied to a Tool Handle. International Journal of Industrial Ergonomics, 34 (6), 507-518.

Wiker, SF, Chaffin, DB, and Langolf, GD. 1990, Shoulder Postural Fatigue and Discomfort. International Journal of Industrial Ergonomics, 5, 133-146.

Wiker, SF, Hershkowtiz, E, and Zik, J. 1989, Teleoperator Comfort and Psychometric Stability: Criteria for Limiting Master-Controller Forces of Operation and Feedback During Telemanipulation Proceedings of the National Aeronautics \& Space Administration's Conference on Space Telerobotics. (Jet Propulsion Laboratory, California Institute of Technology., Pasadena, CA).

Wiktorin, C, Selin, K, Ekenvall, L, Kilbom, Å, and Alfredsson, L. 1996, Evaluation of Perceived and Self-Reported Manual Forces Exerted in Occupational Materials Handling. Applied Ergonomics, 27 (4), 231-239. 
A P P E N D I X A : P R O T O C O L A P P R O V A L NOTICES F R M N I O S H N D W V U 
Date June 24, 2005

From Chair, NIOSH HSRB

Subject HSRB 05-HELD-02XP "The Effects of Vibration and Force Level on the Accuracy of Grip and Push Force Recall"

To Thomas W. McDowell

Research Engineer, PERT, ECTB, HELD

Through: Team Leader, PERT, ECTB, HELD

Chief, ECTB, HELD

Director, HIELD

\section{General Comments and IRB Actions}

I have reviewed the subject protocol using the expedited procedure in that it presents no more than minimal risk and involves a human factors evaluation (estimation of grip force under varying conditions of simulated routine tool vibration) as provided for in criterion \#7 in 45CFR 46.110. The protocol is approved and you are authorized to begin data collection.

Parenthetically, I note that on pg 10 of the protocol, "Benefits to Study Participants" section you have a sentence which states "Other than the reimbursement for their participations, there are no known benefits for the test subjects for participating in the study." For future reference, the reimbursements to study participants should not be considered a benefit of being in the study as this payment is merely intended to compensate them for lost time and expenses associated with their participation. It should not be thought of as a gain or incentive for participation.

I would also like to acknowledge that the protocol, consent document, and supporting materials were well organized and clearly presented. I would like to thank you for making the review process easier. This protocol is granted approval for another year (6/24/2006). However, if you make any substantive changes to the protocol, please notify me immediately.

Protocol Issues - None

Consent Form Issues - None

Addenda Issues (Scripts, questionnaires, brochures, etc.) - None

End of report

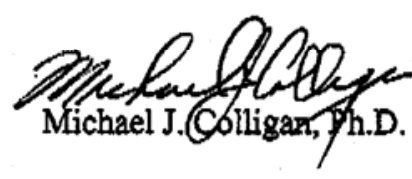

cc:

HSRB 05-FELDD-02XP 


\section{McDowell, Thomas}

From: $\quad$ Charles Craig [CCraig@hsc.wvu.edu]

Sent:

Monday, November 14, 2005 1:52 PM

To:

McDowell, Thomas

Cc:

Lilo.Ast@mail.wvu.edu

Subject:

Research protocol

Hi. Thanks for your responses to our inquiries. The WVU IRB will accept the approval by the NIOSH IRB and it will not be necessary for you to file with us. You may proceed with your study according to the instructions you received from NIOSH. We will make a notation that your study has been approved by the NIOSH IRB, in case we need that information. Sincerely, CRCraig

Charles R. Craig, Ph.D.

Director, Research Compliance 
A P P E N D I X B : R E C R U I T I N G A N N O U N C E M E N T 


\section{WANTED \\ VOLUNTEERS FOR A NIOSH STUDY MEN AND WOMEN AGES 18 AND OVER}

The National Institute for Occupational Safety and Health (NIOSH) located at 1095 Willowdale Road in Morgantown is recruiting volunteers to be test subjects in a research study. This study is designed to examine the effects of vibration on one's ability to duplicate handgrip forces applied to a handle.

\section{What Is Required?}

The tests involve grasping a metal handle with one hand and applying a specified grasping force. The handle may be still, or it may be vibrating as you attempt to maintain the target force. The grasping forces will be between 5 and 30 pounds. Your force will be displayed on a computer monitor while you try to "memorize" the force. Then, you will rest for a short period. Next, the force display will be shielded from your eyes, and you will be asked to grasp the handle and duplicate the force to the best of your ability. At the end of each trial, you will be given another short rest period. There may be a total of up to 48 trials per testing session. The total test session will be less than 3 hours. You may be asked to take part in more than one session, but you will not take part in more than one session in a 24-hour period. You don't have to take part in more than one test session if you don't want to.

\section{Reimbursement and Freedom to Withdraw}

To repay you for your time, you will be reimbursed $\$ 45.00$ for each test session that you complete. You will be reimbursed after each session. You will be free to withdraw from the study at anytime for any reason. If you withdraw before a session is complete, you will be reimbursed on a pro-rated basis at the rate of $\$ 15.00 /$ hour.

\section{Possible Risks}

We do not expect that you will be harmed in any way by taking part in this study. This type of testing is routinely done in several labs around the world. The amount of vibration that you will feel will be similar to the vibration you would feel when using a hand-held electric drill or mowing a lawn. Although no adverse health effects are anticipated, there exists a possibility that you may feel minor tingling in your hand and fingers after each trial, but this tingling should not last more than a few seconds. There is also a slight chance of some minor muscle soreness in your hand or forearm, but this soreness should not last more than 24 hours and will resolve itself without any medical treatment.

\section{Who Is Eligible?}

You must be at least 18 years of age and in generally good health. If you have ever experienced tingling, numbness or appearance of white patches in your hands for a notable duration, you are not eligible to participate.

\section{What If I Am Interested?}

For more information call Thomas McDowell at (304) 285-6337, Monday through Friday, 7:00 AM to 3:00 PM. 
A P P E N D I X C: S U B J E C T C ON S E T T F O R 


\author{
NATIONAL INSTITUTE FOR OCCUPATIONAL SAFETY AND HEALTH (NIOSH) \\ CENTERS FOR DISEASE CONTROL \\ U.S. PUBLIC HEALTH SERVICE \\ U.S. DEPARTMENT OF HEALTH AND HUMAN SERVICES
}

\author{
CONSENT TO PARTICIPATE IN A RESEARCH STUDY
}

You have been asked to participate in a NIOSH research study. We explain here the nature of your participation, describe your rights, and specify how NIOSH will treat your records.

\title{
I. DESCRIPTION OF THE STUDY
}

1. Title:

\author{
THE EFFECTS OF VIBRATION AND FORCE LEVEL ON THE ACCURACY OF \\ GRIP AND PUSH FORCE RECALL
}

\section{Sponsor}

This study is a part of our ongoing research efforts to enhance safety of workers operating hand-held power tools. The study is being conducted by the Engineering and Control Technology Branch (ECTB) in the Health Effects Laboratory Division (HELD) of the National Institute for Occupational Safety and Health (NIOSH) in Morgantown, West Virginia.

Project Officer: Dr. Ren Dong, NIOSH

\section{Purpose \& Benefits}

Workers who use vibrating tools often have problems with their fingers and hands. One reason for this might be that workers have to use extra force or strength when they grip and use power tools such as saws, drills and grinders. This extra tension may lead to increased vibration exposure and more hand and arm problems. It is hard to measure muscle strain when people are using real tools. Another way is to see if people can match the amount of force they use with a real tool when they grip a measuring tool in our lab. If people are good at matching this force, it will help us to study the effects of vibration on the hands and arms in a lab setting. This information may help us to design better tools. There won't be any immediate benefits to you for being in this study, but what we learn may help us to develop safer tools in the future.

\section{CONDITIONS OF THE STUDY}

\section{Test Method}

We will conduct a series of tests designed to judge the impact of various work factors on a human's ability to match grip and push forces used during the use of power tools. The general test setup and subject posture are shown in Figure 1. You will be asked to grasp a metal handle and apply specific grip and push forces. You will be able to see your applied forces on a computer display as you try to "memorize" your grip and push forces. The handle may be still, or it may be vibrating. You will then release the handle and take a short rest. After the rest period, you will be asked to grasp the handle again and try to "match" the memorized forces. You will not be able to see your applied grip and 
push forces during this time. After your matching attempt, you will take another short rest. You will repeat this process several times. Sometimes, the handle will vibrate faster or slower than the previous trial.

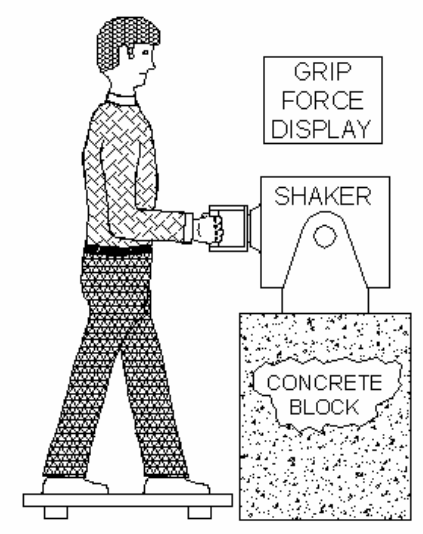

Figure 1. Typical Test Posture

There may be a total of up to 40 trials per testing session. The total test session will be less than 3 hours. You may be asked to take part in more than one session, but you will not take part in more than one session in a 24-hour period. You don't have to take part in more than one test session if you don't want to.

\section{Risks or Discomfort}

We do not expect any risks to you for being in the study. This type of testing is done in several labs around the world. The amount of vibration that you will feel will be similar to the vibration you would feel when using a hand-held electric drill or while mowing your lawn.

It is possible that you may feel some tingling in your hand and fingers after each trial, but this tingling should not last more than a few seconds. There is also a slight chance that your hand and/or forearm may become sore the day following the test. This soreness will not require medical treatment and will not last more than a day.

\section{Medical Care}

Injury or harm from this project is unlikely, but if it results, medical care is not provided, other than emergency treatment. If you are injured through negligence of a NIOSH employee you may be able to obtain compensation under Federal Law. If you want to file a claim against the Federal government your contact point is: Public Health Service Claims Office: (301) 443-1904. If you are injured through the negligence of a NIOSH contractor, your claim would be against the contractor, not the federal government. If think you have been injured by being in this study, you also should contact Ren Dong, the study's project officer, at 304-285-6332.

\section{Contact Information}

If you have any questions about this research, contact Ren Dong, the study's project officer, at 304285-6332. If you have any questions about your rights as a member of the study, contact Mike Colligan, chair of the NIOSH Human Subjects Review Board, at 513-533-8222.

\section{Reimbursement/ Right to Refuse or Withdraw}

For your time, you will receive a check for $\$ 45.00$ for each session that you complete. You will receive a check after each session. Your participation in this study is strictly voluntary and you may 
leave the study at any time for any reason. However, if you leave the study before the session is completed, you will be reimbursed on a pro-rated basis at a rate of $\$ 15.00 /$ hour.

\section{USE OF INFORMATION}

This study is being done by The National Institute for Occupational Safety and Health (NIOSH). $\mathrm{NIOSH}$ is part of the Centers for Disease Control (CDC), a government agency in the Department of Health and Human Services. We collect this information in order to learn about various kinds of work hazards that may influence the health of the American worker.

NIOSH is allowed to collect and keep information about you, including your results from this study, along with your social security number (if applicable), because of three laws passed by Congress. These laws are:

1. The Public Health Service Act (42 U.S.C 241)

2. The Occupational Safety and Health Act (29 U.S.C. 669)

3. The Federal Mine Safety and Health Act of 1977 (30 U.S.C. 951)

You will decide whether you want to provide us with this information by being in this study. You are free to choose not to be in this study. It is up to you. If the information we are collecting is maintained and retrieved by personal identifiers, such as your name and social security number, it will become part of the CDC record system and we will protect it to extent allowed by law. You should know, however, that there are conditions under the Privacy Act when we could be authorized to release this information to outside sources. These conditions under which we might release this information are listed in the Appendix (the Privacy Act).

\section{SIGNATURES}

I have read this consent form and I agree to participate in this study.

Participant

Age

Date

(Signature)

I, the NIOSH representative, have accurately described this study to the participant.

Representative

Date

(Signature) 


\section{Appendix}

The Information you provide will become part of the CDC Privacy Act System, 09-20-0147, "Occupational Health Epidemiological Studies and EEOICPA Program Records" and may be disclosed to

- Appropriate state or local heath departments to report communicable diseases;

- A State Cancer Registry to report cases of cancer where the state has a legal reporting program providing for confidentiality;

- Private contractors assisting $\mathrm{NIOSH}$;

- Collaborating researchers under certain circumstances to conduct further investigations;

- One or more potential sources of vital statistics to make determinations of death, health status or to find last known address;

- The Department of Justice or the Department of Labor in the event of litigation;

- Congressional offices assisting an individual in locating his or her records;

- The Department of Justice to assist in determining the eligibility for compensation to uranium workers or their survivors [optional but must be used if study pertains to uranium workers]

You may request an accounting of the disclosures made by $\mathrm{NIOSH}$.

Except for these and other permissible disclosures authorized by the Privacy Act, or in limited circumstances required by the Freedom of Information Act, no other disclosures may be made without your written consent. 
A P P E N D I X D : S U B J E C T I N S T R U C T I O N S E T 


\section{Pre-test oral instructions to the subject:}

Workers who operate vibrating power tools on a regular basis may develop problems in their fingers, hands, and arms. They may experience chronic tingling or numbing, and in some extreme cases, the condition may become disabling.

We are trying to develop ways to protect these workers, but we need to know more about the vibration as it enters the worker's hands. The amount of vibration actually entering the worker's hands depends on the pushing and gripping forces the worker uses when using the tools. Therefore, we want to learn about these hand forces. It is difficult to measure these forces in work environments because instruments are expensive and delicate. We are trying to develop a new way to learn about hand forces without having to place instruments on tool handles. We hope that you can help us develop this new method.

\section{(Investigator demonstrates the proper posture and procedure during these instructions.)}

What we are going to ask you to do today is to grip this handle that we have mounted on a shaker. This handle is our way of simulating a vibrating power tool. When we tell you to, you will step on the platform, grasp the handle at its mid-point, and apply grip and push forces to the handle. The handle will usually be vibrating, but sometimes it won't be vibrating. You will try to line up the pointers on the two dial gauges with the targets indicated at the top of each gauge. The gauge on the left is your grip force. The gauge on the right is your pushing force. Try to hold your forces as close to the targets as you can. Concentrate on how it feels to apply the grip and push forces. The most important part of your job is "memorizing" the applied grip and push forces while you try to maintain the target forces. Please do not talk during this time. After 45 seconds of memorization, I will instruct you to let go of the handle but remain on the platform. The vibration will stop; the dials on the screen will go blank. There will be a short rest period, and then you will try to apply the same amount of grip and push force that you applied during the 45 seconds of memorization. You will re-grip the handle on the "ready" signal and apply the grip and push forces on the "go" signal. When you think you have matched the grip and push forces, I want you to nod your head and hold the grip and push forces steady for another 5 seconds. You will then release the handle, step off the platform, and rest for 90 seconds while we prepare for the next trial. The forces and the vibration will change randomly from trial to trial, but your task will always be to memorize the grip and push forces as you line up the pointers with the targets on the screen.

\section{Reinforce instructions after each 5-minute break:}

Remember to grip the handle in the middle on each trial. Try to line up the pointers with the targets on top of each dial. Memorize the grip and push forces as you hold the forces as steady as you can. After 45 seconds, I'll ask you to release the handle. Remain on the platform. The vibration will stop; the dials on the screen will go blank. There will be a short rest period. Re-grip the handle on the "ready" signal and apply the grip and push forces on the "go" signal. Try your best to apply the same amount of grip and push force that you applied during memorization. When you think you have matched the forces, I want you to nod your head and maintain the grip and push forces for another 5 seconds. On the "release" signal, step off the platform and rest for 90 seconds. 
A P P E N D I X E : C U R R I C U L U M V I T A E 


\title{
Education
}

\author{
Ph.D. Candidate - Occupational Safety \& Health \\ West Virginia University, College of Engineering and Mineral Resources, Morgantown, WV \\ 4.0 G PA ; D egree ex pected May, 2006
}

M.S. - Safety Management, August, 1997

West Virginia University, College of Engineering and Mineral Resources, Morgantown, WV

4.0 G PA ; E arned the 0 utstanding Student A ward for the SE M graduating class of A ugust, 1997.

B.S. - Electrical Engineering Technology, D ecember, 1983 - cum laude

Fairmont State College (now Fairmont State University), Fairmont, WV

$3.25 \mathrm{G} \mathrm{PA}$; E lected to E psilon Pi Tau, International Technology H onorary

\section{Work Experience}

\section{National Institute for Occupational Safety and H ealth, Morgantown, WV (1/ 1999 - present)}

10/ 2001 - present: Research E ngineer, E ngineering \& C ontrol Technology Branch, H ealth E ffects L ab D ivision

I have assumed a prominent role in the design of study and research protocols and matrices, the design and implementation of instrumentation, engineering apparatus, and other technologies, and participating in the collection, reduction, and analysis of data associated with the study of hand-arm vibration (HAV) and other related occupational safety and health research projects. I am responsible for translating conceptual test and measurement scenarios into appropriate experimental protocols, matrices, and data acquisition system designs. I design and develop new experiments and instrumentation systems and/ or propose modifications or adaptations to existing technologies for the purpose of (a) measuring, monitoring, or characterizing workers' physical, physiological, and/ or sensory responses and performances, and (b) evaluating or validating new experimental designs and technologies as well as the efficacy of proposed engineering solutions. I participate in the day-to-day operation of the HAV laboratory and the testing undertaken therein.

I collaborate with other scientists and peers in the preparation and presentation of manuscripts for dissemination through peer-reviewed journals, NIO SH publications, conference presentations, and other appropriate media. I identify and recommend methods for preventing injuries and illnesses in the workplace. I develop information to support recommendations for revising existing standards and guidelines or developing new guidelines or standards pertaining to the reduction of hand-arm vibration syndrome (HAVS) and related occupational diseases, disorders, and illnesses.

I maintain an in-depth knowledge of current research and scientific information to keep abreast of the latest developments in areas pertaining to HAV, ergonomics, biomechanics, musculoskeletal disorders, and other occupational diseases, disorders, and illnesses. I conduct literature searches and reviews to identify the latest pertinent experimental methods, technologies, and data acquisition techniques available to measure or monitor mechanical, biomechanical, physiological, sensory, and performance variables. I use this knowledge to evaluate and improve existing studies and protocols, to develop new research studies and protocols, and to upgrade laboratory equipment, practices, and procedures needed to complete state-of-the-art occupational health and safety research experiments. 


\section{T. W. McDowell}

\section{NIOSH (continued)}

1/ 1999 - 10/ 2001: G eneral E ngineer, Respirator Branch, D ivision of Respiratory D isease Studies

Duties included evaluation and laboratory testing of numerous self-contained breathing apparatus (SCBA) as a part of fire fighter fatality and injury investigations. Produced several comprehensive reports on these investigations that included inspection findings, performance test protocols, data tables, data analyses, photographs, conclusions, and recommendations to the fire departments involved. The investigations required extensive interaction with fire department chiefs and other department personnel, fire marshals, arson investigators, union representatives, and SCBA manufacturers' representatives. Traveled with and assisted the D ivision of Safety Research Fire Fighter Fatality Investigation and Prevention Team on fatality investigations.

Other assignments included the collection and documentation of evidence and the production of detailed written reports on investigations of SCBA problems reported to NIOSH by industrial and fire service representatives. Worked with engineers from respirator manufacturers, representatives from other government agencies such as OSHA, the U.S. D epartment of Transportation, and the Food and D rug Administration, as well as with internationally recognized forensic scientists and investigators. Was responsible for writing all official correspondence, maintaining the Field Problems database records, and managing the official files as a part of these investigations. Was responsible for assisting in the development of NIO SH Respirator User Notices, respirator manufacturer's Safety Bulletins, and other publications to alert respirator users to potential safety hazards and necessary precautions.

Traveled to several fire departments to perform audits of their SCBA maintenance programs. The fire department programs were evaluated and compared to recognized national standards including NFPA 1500, NFPA 1404, OSHA 1910.134, and ANSI Z-88.2. D eveloped an interview questionnaire and checklist to ensure consistent and comprehensive evaluations of these fire department programs. Reported my findings and made recommendations for improvements. Excerpts from applicable standards were included with each recommendation. One of these audits led to the publication of an article in the July 2000 issue of Fire E ngineering magazine. This article, which endorsed the NIO SH program, led to dozens of requests from fire departments who wished to upgrade their SCBA maintenance programs.

Laboratory responsibilities included the development of test procedures for evaluating the performance of SCBA, testing SCBA as a part of investigations, videotaping all SCBA inspections and performance tests, and documenting and reporting my inspection and test results. Designed new SCBA testing systems, specified instrumentation and other lab equipment, solicited bids for the purchase of lab equipment, scheduled periodic instrument calibrations and maintenance, and managed all other functions required for the efficient operation of the Firefighter SCBA Evaluation lab. Was also responsible for all record-keeping, laboratory safety, and laboratory hygiene.

Was involved in the budget management for the Firefighter SCBA Evaluation project. Made all appropriate purchases for the lab and in support of the project. Also completed requisition requests and sole-source justifications when appropriate. Managed expenditures totaling approximately $\$ 25,000$ each fiscal year. Served as the Technical Monitor for a D ivision of Safety Research-funded $\$ 41,000$ project to produce software for fire departments for their use in developing and managing SCBA maintenance programs. Completed the Basic Project Officer training provided by the D epartment of Health and Human Services. 
TMC Technologies, Inc., Fairmont, WV

(4/ 1996 - 1/ 1999)

Title: E ngineer

Was responsible for the design of instrumentation systems, motor controls, and process controls for a wide variety of industrial applications. D uties also included bid and proposal preparation, equipment specification, and oral (Power Point) and written presentation of detailed technical solutions to customers. Developed and conducted training courses for client's managers, engineers, supervisors, technicians, and operators. Produced hundreds of AutoCAD drawings, electrical schematics, cabinet layouts, process flow charts, etc. Projects included design and code development for MS Visual Basic 5.0 applications for client/ server database management systems.

Mission Operation \& Maintenance, Inc., Grant Town, WV

(5/ 1992 - 2/ 1996)

4/ 1994 - 2/ 1996: Title: Regulatory Complianœ and Safety Specialist/ E ngineer

Managed safety and environmental compliance programs. Coordinated site evaluation and data analysis for determining proper engineering controls, administrative adjustments, and PPE for dust, noise and other industrial hygiene concerns. Conducted injury and near-miss incident investigations and site safety and environmental audits. Served as the facility's Safety Committee chairman. Maintained air emissions and water discharge permits. Completed periodic reports and responded to inquiries from WV DEP and Federal EPA, O SHA, MSHA, and other regulatory agencies. D esigned database forms and macros for agency-required management of daily plant emissions data. D eveloped several plant orders and safety training programs including blood borne pathogens control, hearing conservation, incipient fire control, confined space entry, hazard communication, and many others. Served as instructor for periodic employee safety training.

\section{5/ 1992 - 4/ 1994: Title: Instrumentation \& E lectrical T echnician}

Involved with testing, start-up, and on-line maintenance of a waste-coal fired 80 megawatt power generation facility. Responsible for plant-wide instrumentation and process controls, computer systems, man-machine interfaces, PLCs, and all other instrumentation and electrical systems for fuel excavation and preparation, boiler control, emissions data collection, and all other phases of the generation process. D eveloped and conducted training courses for technicians and operators.

Charlotte-Mecklenburg Utility Dept., Charlotte, NC

(4/ 1986 - 5/ 1992)

Title: Instrumentation \& Controls Technician

D esigned, installed, and maintained a wide variety of process controls and instrumentation systems, flow and level control systems, data acquisition systems, data transmission systems, etc., in all phases of waste water and fresh water treatment operations at eight large treatment facilities. D eveloped and conducted training courses for technicians and operators. Verified and punch-listed contracted projects. 


\section{Peer-Reviewed Joumal Articles}

McDowell, TW, Wiker, SF, D ong, RG, Welcome, DE, and Schopper, AW. (2006). "Evaluation of Psychometric Estimates of Vibratory Hand-Tool Grip and Push Forces," International Joumal of Industrial Ergonomics, 36(2), 119-128.

Dong, RG, Welcome, DE, McDowell, TW, and Wu, JZ. "Measurement of Biodynamic Response of Human Hand-Arm System," Journal of Sound and Vibration. (accepted D ecember 2005)

Dong, RG, McDowell, TW, Welcome, DE. (2005). "Biodynamic Response at the Palm of the Human Hand Subjected to a Random Vibration," Industrial Health, 43(1), 241-255.

Dong, RG, Welcome, DE, McDowell, TW, Wu, JZ, and Schopper, AW. "Frequency Weighting D erived from Power Absorption of Fingers-Hand-Arm System under $\mathrm{Z}_{\mathrm{h}}$-Axis Vibration," Journal of Biomechanics, (in press; available online September 12, 2005)

Dong, RG, McDowell, TW, Welcome, DE, and Smutz, WP. (2005). "Correlations between Biodynamic Characteristics of Human Hand-Arm System and the Isolation Effectiveness of Anti-Vibration Gloves," International Journal of Industrial Ergonomics, 35, 205-216.

Dong, RG, Rakheja, S, McDowell, TW, Welcome, D, Wu, J, Warren, C, Barkley, J, Washington, B, and Schopper, AW. (2005). "A Method for Determining the Effectiveness of Antivibration Gloves Using Biodynamic Responses of the Hand-Arm System," Journal of Sound and Vibration, 282(3-5), 1101-1118.

Dong, RG, Wu, JZ, McDowell, TW, Welcome, DE, and Schopper, AW. (2005) "Distribution of Mechanical Impedance at the Fingers and the Palm of Human Hand," Journal of Biomechanics, 38(5), 1165-1175.

Dong, RG, McDowell, TW, Welcome, DE, Barkley, J, Warren, C, and Washington, B. (2005) "Effects of Hand Coupling Conditions on the Effectiveness of Air Bladder Anti-Vibration Gloves," Journal of Low Frequency Noise, Vibration and Active Control, 23(4), 231-248.

Dong, RG, Wu, JZ, Welcome, DE, and McDowell, TW. (2005) "Estimation of Vibration Power Absorption D ensity in Human Fingers," Journal of Biomechanical Engineering, 127(5), 849-856.

Dong, RG, Welcome, DE, McDowell, TW, Wu, JZ. (2004). "Biodynamic Response of Human Fingers in a Power Grip Subjected to a Random Vibration," Journal of Biomechanical Engineering, 126(4), 447-457.

Dong, RG, McDowell, TW, Welcome, DE, Warren, C, and Schopper, AW. (2004). "An Evaluation of the Standardized Chipping Hammer Test Specified in ISO 8662-2, 1992," Annals of Occupational Hygiene, 48(1), 39-49.

Dong, RG, Schopper, AW, McDowell, TW, Welcome, DE, Wu, JZ, Smutz, WP, Warren, C, and Rakheja, S. (2004) "Vibration Energy Absorption (VEA) in Human Fingers-Hand-Arm System," Medical Engineering \& Physics, 26(6), 483-492.

Dong, RG, McDowell, TW, Welcome, DE, Smutz, WP, Schopper, AW, Warren, C, Wu, JZ, and Rakheja, S. (2003). "On-the-hand Measurement Methods for Assessing Effectiveness of Anti-Vibration Gloves," International Journal of Industrial Ergonomics, 32(4), 283-298.

D ong, RG, McDowell, TW, Welcome, DE, Rakheja, S, Caporali, SA, and Schopper, AW. (2002). "Effectiveness of a Transfer Function Method for Evaluating Vibration Isolation Performance of Gloves When Used With Chipping Hammers," Journal of Low Frequency Noise, Vibration and Active Control, 21(3), 141155. 


\section{Conference Presentations, Book Chapters, and Other Works}

McDowell, TW, Wiker, SF, D ong, RG, and Welcome, DE. (2006). "The Effects of Vibration on Psychophysical Grip and Push Force-Recall Accuracy." (to be presented at the 1st American Conference on Human Vibration, June 5-7, 2006, Morgantown, WV, USA)

McDowell, TW, Wiker, SF, D ong, RG, and Welcome, DE. (2006). "The Measurement of Grip and Push Forces Applied to Vibrating Tools Using a Psychophysical Force-Recall Technique." (to be presented at NORA Symposium 2006, April 18-20, 2006, Washington, DC, USA)

Dong, RG, Welcome, DE, McDowell, TW, Wu, JZ, and Schopper, AW. (2005). "Frequency Weighting Derived From Total Power Absorption of Hand-Arm System," In Proceedings of the 40th United Kingdom Conference on Human Responses to Vibration, Liverpool, UK.

D ong, RG, Wu, JZ, Welcome, DE, McDowell, TW, Krajnak, K, Wirth, O, Brumfield, A, and Schopper, AW. (2005). "NIOSH's Recent Studies on Hand-Transmitted Vibration Exposure and Health Effects," In Proceedings of the 13th Japan Group Meeting on Human Responses to Vibration, O saka, Japan.

McDowell, TW, Dong, RG, and Welcome, DE. (2004). "A Pilot Study of Glove Effects on a Force Matching Method," Abstract in Conference Program \& Preconference Proceedings of the 10th International Conference on Hand-A rm Vibration, Las Vegas, NV, USA, 83-84.

D ong, RG, Warren, C, Welcome, DE, McDowell, TW, and Wu, JZ. (2004). "A Comparison and Evaluation of Reported Mechanical Impedance Datasets of the Human Hand-Arm System," Abstract in Conference Program \& Preconference Proceedings of the 10th International Conference on Hand-Arm Vibration, Las Vegas, NV, USA, 101-102.

Welcome, DE, D ong, RG, McDowell, TW, and Wu, JZ. (2004). "Evaluations of Instrumentation for Measuring Biodynamic Responses of the Hand-Arm System," Abstract in Conference Program \& Preconference Proceedings of the 10th International Conference on Hand-Arm Vibration, Las Vegas, NV, USA, 94-96.

Dong, RG, McDowell, TW, Welcome, DE, and Wu, JZ. (2004). “An Investigation of the Relationship Between Vibration-Induced White Finger and Power Absorption," Abstract in Conference Program \& Preconference Proceedings of the 10th International Conference on Hand-Arm Vibration, Las Vegas, NV, USA, 80-82.

Dong, RG, McDowell, TW, Welcome, DE, Rakheja, S, Smutz, WP, Warren, C, Wu, JZ, and Schopper, AW. (2003) "Effectiveness of Anti-Vibration Gloves," Paper No. 18. Abstract in NORA Symposium 2003: Working Partnerships: Applying Research to Practice, Arlington, VA, USA, 45.

D ong, RG, Wu, JZ, McDowell, TW, Welcome, DE, and Schopper, AW. (2003). "Vibration Energy Transmitted from a Simulated Tool Handle to Human Fingers and the Palm of the Hand," In Proceedings of the 38th United Kingdom Conference on Human Responses to Vibration. Institute of Naval Medicine, Alverstoke, G osport, UK, 455-460.

D ong, RG, McDowell, TW, Welcome, D, Smutz, WP, Warren, C, Wu, JZ, Rakheja, S, and Schopper, AW. (2002). "Assessing Vibration Isolation Performance of Gloves Using On-Hand Measurement Methods," In Proceedings of the 37th United Kingdom Conference on Human Responses to Vibration. Loughborough, UK: Loughborough University, 130-141. 
Dong, RG, McDowell, TW, Welcome, D, Wu, J, Warren, C, Smutz, WP, and Schopper, AW. (2002). "Mechanical Energy Absorption in Human Fingers Exposed to Hand-Transmitted Vibration," In J. Vossoughi (Ed.), Biomedical Engineering: Recent Developments. Washington, DC: Medical and Engineering Publishers, Inc., 159-160.

Dong, RG, McDowell, T, Welcome, D, Wu, J, Schopper, AW, and Rakheja, S. (2002). "Evaluations of a Transfer Function Method for Predicting Vibration Isolation Performance of Gloves," Abstract in Proceedings of the 9th International Congress on Sound and Vibration, ICSV9. O rlando, FL: University of Central Florida, 80.

Dong, RG, Welcome, D, McDowell, T, Schopper, AW, and Tubbs, RL. (2002). NIOSH Health Hazard Evaluation Report: HETA \#2001-0073-2869, City of Cincinnati Sewers, Water Works \& Public Services, Cincinnati, O hio - March 2002. Cincinnati: National Institute for O ccupational Safety \& Health.

Winn, GL and McDowell, T. (1999). "Waiting to Exhale: The Pros and Cons of Alcohol Testing Technologies," Security Management, 43(9), 129-133. 